TRANSACTIONS OF THE

AMERICAN MATHEMATICAL SOCIETY

Volume 356, Number 2, Pages 509-555

S 0002-9947(03)03398-1

Article electronically published on September 22, 2003

\title{
SIMPLE BIRATIONAL EXTENSIONS OF THE POLYNOMIAL ALGEBRA $\mathbb{C}^{[3]}$
}

\author{
SHULIM KALIMAN, STÉPHANE VÉNÉREAU, AND MIKHAIL ZAIDENBERG
}

\begin{abstract}
The Abhyankar-Sathaye Problem asks whether any biregular embedding $\varphi: \mathbb{C}^{k} \hookrightarrow \mathbb{C}^{n}$ can be rectified, that is, whether there exists an automorphism $\alpha \in$ Aut $\mathbb{C}^{n}$ such that $\alpha \circ \varphi$ is a linear embedding. Here we study this problem for the embeddings $\varphi: \mathbb{C}^{3} \hookrightarrow \mathbb{C}^{4}$ whose image $X=\varphi\left(\mathbb{C}^{3}\right)$ is given in $\mathbb{C}^{4}$ by an equation $p=f(x, y) u+g(x, y, z)=0$, where $f \in \mathbb{C}[x, y] \backslash\{0\}$ and $g \in \mathbb{C}[x, y, z]$. Under certain additional assumptions we show that, indeed, the polynomial $p$ is a variable of the polynomial ring $\mathbb{C}^{[4]}=\mathbb{C}[x, y, z, u]$ (i.e., a coordinate of a polynomial automorphism of $\left.\mathbb{C}^{4}\right)$. This is an analog of a theorem due to Sathaye (1976) which concerns the case of embeddings $\mathbb{C}^{2} \hookrightarrow \mathbb{C}^{3}$. Besides, we generalize a theorem of Miyanishi (1984) giving, for a polynomial $p$ as above, a criterion for when $X=p^{-1}(0) \simeq \mathbb{C}^{3}$.
\end{abstract}

\section{INTRODUCTION}

Generalizing a theorem of A. Sathaye [34] it is proven in [19] that if a surface $X=p^{-1}(0) \subseteq \mathbb{C}^{3}$ with $p=f u+g \in \mathbb{C}^{[3]}$ and $f, g \in \mathbb{C}[x, y]$ is acyclic (that is, $\left.\widetilde{H}_{*}(X ; \mathbb{Z})=0\right)$, then $p$ is a variable of the polynomial ring $\mathbb{C}^{[3]}$, i.e., a coordinate of an automorphism $\alpha \in$ Aut $\mathbb{C}^{3}$. Thus $X$ can be rectified, and so is isomorphic to $\mathbb{C}^{2}$. This does not hold any more in $\mathbb{C}^{4}$ (even with $f \in \mathbb{C}[x]$ ). Indeed [15] the Russell cubic 3-fold

$$
X:=\left\{p=x^{2} u+x+y^{2}+z^{3}=0\right\} \subseteq \mathbb{C}^{4}
$$

is an exotic $\mathbb{C}^{3}$, i.e., is diffeomorphic to $\mathbb{R}^{6}$ and non-isomorphic to $\mathbb{C}^{3}$. In 2.28, 3.21 and 3.6 below we give a criterion for when a 3 -fold $X=p^{-1}(0) \subset \mathbb{C}^{4}$ with

$$
p=f(x, y) u+g(x, y, z) \in \mathbb{C}^{[4]} \quad\left(f \in \mathbb{C}^{[2]} \backslash\{0\}, g \in \mathbb{C}^{[3]}\right)
$$

is acyclic (resp., is isomorphic to $\mathbb{C}^{3}$, resp., is an exotic $\mathbb{C}^{3}$ ). In particular, we show in 2.11 that if $X$ is acyclic, then actually it is diffeomorphic to $\mathbb{R}^{6}$. If furthermore (3.21) $X$ is isomorphic to $\mathbb{C}^{3}$, then any fiber $X_{\lambda}:=p^{-1}(\lambda)(\lambda \in \mathbb{C})$ of the polynomial $p$ is isomorphic to $\mathbb{C}^{3}$ as well, and moreover (with an appropriate choice of coordinates $(x, y))$ all fibers of the morphism

$$
\rho:=(x, p): \mathbb{C}^{4} \rightarrow \mathbb{C}^{2}
$$

Received by the editors December 5, 2001.

2000 Mathematics Subject Classification. Primary 14R10, 14R25.

Key words and phrases. Affine space, polynomial ring, variable, affine modification, birational extension.

The research of the first author was partially supported by the NSA grant MDA904-00-1-0016.

The third author is grateful to the IHES and to the MPI at Bonn (where a part of the work was done) for their hospitality and excellent working conditions. 
are reduced and isomorphic to $\mathbb{C}^{2}$. We do not know whether in that case a polynomial $p$ in (11) must be a variable of the polynomial ring $\mathbb{C}^{[4]}$, and $\rho$ must be a trivial family. However, in section 4 in many cases we provide affirmative answers to these questions and give simple concrete examples where the answers remain unknown. By virtue of the Quillen-Suslin Theorem and the Bass-Connell-Wright Theorem [2] (which implies that any $\mathbb{C}^{n}$-fiber bundle over an affine base is in fact a $\mathbb{C}^{n}$-vector bundle), the latter question would be answered in positive if the following conjecture [7, (3.8.5)] (cf. [35, 20) were true for $n=2=\operatorname{dim} S$ :

Dolgachev-Weisfeiler Conjecture. Let $f: X \rightarrow S$ be a flat affine morphism of smooth schemes with every fiber isomorphic (over the residue field) to an affine space $\mathbb{A}_{k}^{n}$. Then $f$ is locally trivial in the Zariski topology (i.e., is a fiber bundle).

Whereas the former question (as whether $p$ is a variable of the polynomial ring $\mathbb{C}^{4}$ ) is a particular case (with $n=4$ and $k=3$ ) of the famous

Abhyankar-Sathaye Embedding Problem. Is it true that any biregular embedding $\mathbb{C}^{k} \hookrightarrow \mathbb{C}^{n}$ is rectifiable, i.e., is equivalent to a linear one under the action of the group Aut $\mathbb{C}^{n}$ on $\mathbb{C}^{n}$ ?

Geometrically, the situation can be regarded as follows (cf. [19]). The morphism $\sigma:(x, y, z, u) \longmapsto(x, y, z)$ represents the 3 -fold $X$ as a birational modification of $Y:=\mathbb{C}^{3}$. The latter essentially consists of replacing the divisor $D:=D(f) \subseteq Y$ by the exceptional one $E:=\{f=g=0\} \subseteq X$. All the important properties of $X$ can be recovered in terms of the restriction $\left.\sigma\right|_{E}: E \rightarrow D$. In our setting both $E$ and $D$ are cylindrical surfaces, namely $E=C \times \mathbb{C}$ and $D=\Gamma \times \mathbb{C}$, where $C:=D(f) \cap D(g) \subset Y$ is the center of the blowing up $\sigma$ and $\Gamma:=f^{-1}(0) \subseteq \mathbb{C}_{x, y}^{2}$. This makes it possible to formulate the criteria mentioned above in terms of the natural projection $\pi: C \rightarrow \Gamma,(x, y, z) \longmapsto(x, y)$, and enables us in concrete examples to verify these criteria.

Let us briefly describe the content of this paper whose summary appeared in ([17]). Section [1 contains preliminaries; it can be omitted at the first reading and consulted when necessary. However, some results obtained here (and used later on in the proofs) are of independent interest. For instance, this concerns 1.3 where we treat the question of when a birational extension of a UFD is again a UFD. Furthermore, generalizing an observation due to V. Shpilrain and J.-T. Yu [36 37 . we claim in $1.32-1.33$ that for arbitrary polynomials $p, q \in \mathbb{C}\left[x_{1}, \ldots, x_{n}\right][y]$ the hypersurfaces in $\mathbb{C}^{n+1}$ given respectively by the equations

$$
y=q(p(y)) \quad \text { and } \quad y=p(q(y))
$$

are isomorphic and moreover, 1-stably equivalent (see 4.23). We also use the fact (see 1.12) that a one-point compactification of an acyclic smooth affine variety is a homology manifold which is a homology sphere and satisfies the Alexander duality.

In section 2 we study the topology of the 3 -folds $X$ as above. More generally, we work with a 3 -fold $X=\{p=f u+g=0\} \subset Y \times \mathbb{C}$, where $f, g \in \mathbb{C}[Y]$ with $Y$ being a smooth acyclic affine 3 -fold and $D:=f^{-1}(0) \subseteq Y$ being a cylinder $D=\Gamma \times \mathbb{C}$ over an affine curve $\Gamma$ (whereas in subsection $[2.3 Y$ itself is supposed to be a cylinder over an acyclic affine surface $Z$, i.e., $Y=Z \times \mathbb{C}$, with $\Gamma \subseteq Z$ ). The main results of section 2 (see 2.11, 2.27) and 2.28) provide a criterion for when such a 3 -fold $X$ is diffeomorphic to $\mathbb{R}^{6}$. 
In subsection 3.1 we determine when $X=p^{-1}(0) \subseteq \mathbb{C}^{4}$ with $p=f u+g$ as in (1) is an exotic $\mathbb{C}^{3}$. The main tool used here is Derksen's version of the Makar-Limanov invariant [23, 5] described in subsection [1.3.

Subsection 3.2 is devoted to a study of embeddings $\mathbb{C}^{3} \hookrightarrow \mathbb{C}^{4}$ given by an equation $p=f u+g=0$ with $f \in \mathbb{C}^{[2]} \backslash \mathbb{C}$ and $g \in \mathbb{C}^{[3]}$. In 3.21 we show that in appropriate new coordinates in the $(x, y)$-plane, the $x$-coordinate restricted to any fiber of $p$ gives a $\mathbb{C}^{2}$-fibration. On the other hand, the restriction of $p$ to any hyperplane $x=$ const is a variable of the polynomial ring $\mathbb{C}[y, z, u]$ (in the latter case we say in brief that $p$ is an $x$-residual variable).

A complete analog of Theorem 7.2 in [19] cited at the beginning holds if the polynomial $p \in \mathbb{C}^{[4]}$ is linear with respect to two (and not just one) variables. Indeed (3.24) if the 3 -fold $X$,

$$
p=a(x, y) u+b(x, y) v+c(x, y)=0,
$$

in $\mathbb{C}^{4}$ is smooth and acyclic, then $p \in \mathbb{C}^{[4]}$ is a variable. We give a simple criterion (in terms of the coefficients $a, b, c \in \mathbb{C}[x, y]$ ) for when this is the case.

In section 4 we concentrate on the Abhyankar-Sathaye Problem for our particular class of embeddings. The main results 4.2, 4.16 of subsection 4.1 provide sufficient conditions for when an $x$-residual variable $p=f u+g \in \mathbb{C}^{[4]}$ as in (1) is indeed a variable. For instance (see 4.2 4.3) this is the case if $\operatorname{deg}_{z} g \leq 1$, or $f$ is a power of an irreducible polynomial, or else $f \in \mathbb{C}[x]$ (the latter result strengthens those of M. Miyanishi [28, Thm. 2], where it is supposed in addition that $g \in \mathbb{C}[y, z])$. As another example, we show (see 4.17) that the polynomials

$$
p_{1}:=x y^{2} u+y+x z+x y z^{2} \quad \text { and } \quad p_{3}:=x y^{2} u+y+x^{2} z+x^{3} y z^{2}
$$

are variables of $\mathbb{C}^{[4]}$. However, we do not know whether or not

$$
p_{2}:=x y^{2} u+y+x^{2} z+x y z^{2}
$$

is also (whereas $p_{2}^{-1}(\mu) \simeq \mathbb{C}^{3} \forall \mu \in \mathbb{C}$ and $p_{2}$ is an $x$-residual variable and a $\mathbb{C}(x)$ variable; see 4.9 and 4.20 .

In subsection 4.18 (attributed to the second author) we generalize a theorem of D. Wright 41] which says that Sathaye's Theorem holds for the embeddings $\mathbb{C}^{2} \hookrightarrow \mathbb{C}^{3}$ given by an equation $p=f u^{n}+g=0$ with $f, g \in \mathbb{C}[x, y]$ and $n \in \mathbb{N}$. Namely, 4.19 asserts that an $x$-residual variable of the form $p=f u^{n}+g \in \mathbb{C}^{[4]}$, where $f, g \in \mathbb{C}[x, y, z]$ and $n \geq 2$, actually is an $x$-variable.

In the last subsection 4.2 we establish (see 4.25) that every embedding $\mathbb{C}^{3} \hookrightarrow \mathbb{C}^{4}$ given by an equation $p=f u+g=0$ as in (1) can be rectified in $\mathbb{C}^{5}$.

\section{Preliminaries}

1.1. Affine modifications of UFD's. We start by recalling the notion of affine modification [19]; at the same time, we introduce the notation that will be used throughout the paper.

Notation 1.1. Let $Y$ be a reduced, irreducible affine variety over $\mathbb{C}, A=\mathbb{C}[Y]$ be the algebra of regular functions on $Y, I \subseteq A$ be a non-trivial ideal and $f \in I$ be a non-zero element of $I$. The affine modification of the variety $Y$ along the divisor $D_{f}=f^{*}(0)$ with center $I$ is the affine variety $X=\operatorname{spec} \mathrm{A}^{\prime}$, where

$$
A^{\prime}:=A[I / f]=\left\{a^{\prime}=a_{k} / f^{k} \mid a_{k} \in I^{k}\right\} \subseteq \operatorname{Frac} A
$$


The inclusion $A \hookrightarrow A^{\prime}$ corresponds to a birational morphism $\sigma: X \rightarrow Y$ with the exceptional divisor $E=\sigma^{-1}(D)=(f \circ \sigma)^{-1}(0) \subseteq X$, where $D:=\operatorname{supp} D_{f}$. The restriction $\sigma \mid(X \backslash E): X \backslash E \rightarrow Y \backslash D$ is an isomorphism.

Let $D=\bigcup_{i=1}^{n} D_{i}$, resp., $E=\bigcup_{i=1}^{n^{\prime}} E_{i}$, be the decomposition into irreducible components, which we assume to be Cartier divisors. Letting

$$
\sigma^{*}\left(D_{i}\right)=\sum_{j=1}^{n^{\prime}} m_{i j} E_{j}, \quad i=1, \ldots, n,
$$

we consider the $n \times n^{\prime}$ multiplicity matrix $M_{\sigma}=\left(m_{i j}\right)$ with non-negative integer entries. Clearly, $m_{i j}>0 \Leftrightarrow \sigma\left(E_{j}\right) \subseteq D_{i}$.

The following simple observation will be useful in 1.4 below. We denote reg $E_{j}=$ $E_{j} \backslash \operatorname{sing} E_{j}$ and $\operatorname{reg} D_{i}=D_{i} \backslash \operatorname{sing} D_{i}$.

Lemma 1.2. In the notation as above, suppose that the affine varieties $X$ and $Y$ are smooth. If $m_{i j}=1$, then $\sigma\left(E_{j}\right) \nsubseteq \subseteq \operatorname{sing} D_{i}$. Moreover $m_{i j}=1$ if and only if $\sigma\left(\operatorname{reg} E_{j}\right) \subseteq \operatorname{reg} D_{i}$ and $\sigma$ sends the analytic discs in $X$ transversal to $E_{j}$ at a point $Q \in \operatorname{reg} E_{j}$ biholomorphically onto analytic discs in $Y$ transversal to $D_{i}$ at the point $P:=\sigma(Q) \in \operatorname{reg} D_{i}$

Proof. We may assume that $m_{i j}>0$, that is, $\sigma\left(E_{j}\right) \subseteq D_{i}$. For a point $Q \in E_{j}$ with the image $P=\sigma(Q) \in D_{i}$, we let $U \ni P$, resp., $V \ni Q$ be a neighborhood such that $D_{i} \cap U=f_{i}^{*}(0)$, resp., $E_{j} \cap V=h_{j}^{*}(0)$, where $f_{i}$, resp., $h_{j}$, is a holomorphic function in $U$, resp., $V$. Then we have

$$
\left.f_{i} \circ \sigma\right|_{V}=h_{j}^{m_{i j}} \cdot h \quad \text { with } \quad h(Q) \neq 0,
$$

which gives the equality of 1 -forms:

$$
d(f \circ \sigma)(Q)=m_{i j} h_{j}^{m_{i j}-1}(Q) h(Q) \cdot d h_{j}(Q) .
$$

Assuming that $Q \in \operatorname{reg} E_{j}\left(\Leftrightarrow d h_{j}(Q) \neq 0\right)$ and $m_{i j}=1$, from (3) we obtain

$$
d(f \circ \sigma)(Q)=h(Q) \cdot d h_{j}(Q) \neq 0,
$$

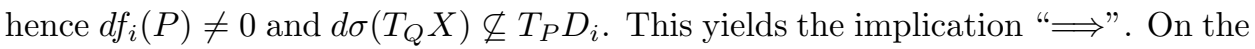
other hand, if $P \in \operatorname{reg} D_{i}\left(\Leftrightarrow d f_{i}(P) \neq 0\right)$ and $d \sigma\left(T_{Q} X\right) \nsubseteq T_{P} D_{i}$, then $d(f \circ \sigma)(Q) \neq$ 0 , and so by (3) $m_{i j}=1$, which gives "£".

For an algebra $B$, denote by $B^{*}$ its group of invertible elements.

Proposition 1.3. In the notation as in 1.1 above, assume moreover that the algebras $A=\mathbb{C}[Y]$ and $A^{\prime}=\mathbb{C}[X]$ are UFD's, and $A^{*}=A^{\prime *}$. Then $n=n^{\prime}$ and the multiplicity matrix $M_{\sigma}$ is unimodular.

Proof. Since the algebras $A$ and $A^{\prime}$ are UFD's, there exist irreducible elements $f_{1}, \ldots, f_{n} \in A$, resp., $h_{1}, \ldots, h_{n^{\prime}} \in A^{\prime}$, such that $D_{i}=f_{i}^{*}(0), i=1, \ldots, n$, resp., $E_{j}=h_{j}^{*}(0), j=1, \ldots, n^{\prime}$. Clearly, $h_{j}=\left(a_{j} / b_{j}\right) \circ \sigma$ with coprime $a_{j}, b_{j} \in A, j=$ $1, \ldots, n^{\prime}$. The regular functions $a_{j}, b_{j}$ do not vanish in $Y \backslash D$, and hence their irreducible factors are proportional to some of the elements $f_{1}, \ldots, f_{n}$. Thus for each $j=1, \ldots, n^{\prime}$ there exists $\gamma_{j} \in A^{*}$ such that

$$
h_{j}=\left(\gamma_{j} \prod_{i=1}^{n} f_{i}^{m_{j i}^{\prime}}\right) \circ \sigma
$$


with $m_{j i}^{\prime} \in \mathbb{Z}$. On the other hand, for each $i=1, \ldots, n$ there exists $\delta_{i} \in A^{\prime *}=A^{*}$ such that

$$
f_{i} \circ \sigma=\delta_{i} \prod_{k=1}^{n^{\prime}} h_{k}^{m_{i k}} .
$$

Plugging successively (4) and (5) into one another and taking into account the assumption that the algebras $A^{\prime}$ and $A$ are UFD's and $\sigma$ is a birational morphism, we obtain the equalities

$$
\begin{aligned}
& \sum_{k=1}^{n^{\prime}} m_{i k} m_{k j}^{\prime}=\delta_{i j}, \quad i, j=1, \ldots, n, \\
& \sum_{i=1}^{n} m_{j i}^{\prime} m_{i k}=\delta_{j k}, \quad j, k=1, \ldots, n^{\prime},
\end{aligned}
$$

that is, $M_{\sigma}^{\prime} \cdot M_{\sigma}=I_{n^{\prime}}$ and $M_{\sigma} \cdot M_{\sigma}^{\prime}=I_{n}$, where $I_{k}$ denotes the identity matrix of order $k$. Hence $n=n^{\prime}$ and $M_{\sigma}^{\prime}=M_{\sigma}^{-1}$, so $M_{\sigma}$ is unimodular.

Proposition 1.4. Suppose that the varieties $X$ and $Y$ as in 1.1 are smooth, and the multiplicity matrix $M_{\sigma}$ is an upper triangular unipotent square matrix:

$$
n=n^{\prime}, \quad m_{i i}=1, i=1, \ldots, n, \quad m_{i j}=0 \quad \forall i>j .
$$

Then $\sigma_{*}: \pi_{1}(X) \rightarrow \pi_{1}(Y)$ is an isomorphism.

Proof. Letting $G=\pi_{1}(Y \backslash D), \quad G^{\prime}=\pi_{1}(X \backslash E)$ and $\sigma^{\prime}=\left.\sigma\right|_{X \backslash E}$, we have that $\sigma_{*}^{\prime}: G^{\prime} \rightarrow G$ is an isomorphism which sends the subgroup

$$
H^{\prime}:=\left\langle\left\langle\alpha_{E_{1}}, \ldots, \alpha_{E_{n}}\right\rangle\right\rangle \subseteq G^{\prime}
$$

into the subgroup

$$
H:=\left\langle\left\langle\alpha_{D_{1}}, \ldots, \alpha_{D_{n}}\right\rangle\right\rangle \subseteq G
$$

where for a hypersurface $Z$ in a complex manifold $X, \alpha_{Z}$ denotes a vanishing loop of $Z$ in $X \backslash Z$, whereas for a group $G$ and elements $a_{1}, \ldots, a_{k} \in G,\left\langle\left\langle a_{1}, \ldots, a_{k}\right\rangle\right\rangle$ denotes the minimal normal subgroup of $G$ generated by $a_{1}, \ldots, a_{k}$. Moreover, $\sigma_{*}: \pi_{1}(X) \rightarrow \pi_{1}(Y)$ is a surjection with kernel ker $\sigma_{*} \cong H / \sigma_{*}^{\prime}\left(H^{\prime}\right)$ (see, e.g., [19] the proof of Prop. 3.1]). Thus we must show that $\sigma_{*}^{\prime}\left(H^{\prime}\right)=H$.

For $i=1, \ldots, n$ denote

$$
H_{i}:=\left\langle\left\langle\alpha_{D_{1}}, \ldots, \alpha_{D_{i}}\right\rangle\right\rangle \subseteq G, \quad \text { resp. }, \quad H_{i}^{\prime}:=\left\langle\left\langle\alpha_{E_{1}}, \ldots, \alpha_{E_{i}}\right\rangle\right\rangle \subseteq G^{\prime},
$$

so that $H_{n}=H$ and $H_{n}^{\prime}=H^{\prime}$. Clearly, $\sigma_{*}^{\prime}\left(\alpha_{E_{1}}\right) \sim \alpha_{D_{1}}$ (where $\sim$ stands for conjugation), hence $\sigma_{*}^{\prime}\left(H_{1}^{\prime}\right)=H_{1}$. We show by induction that $\sigma_{*}^{\prime}\left(H_{i}^{\prime}\right)=H_{i}$ for all $i=1, \ldots, n$.

Assume that this equality holds for $i=k-1<n$. As $m_{k k}=1$, by 1.2 we may conclude that for a general point $Q \in E_{k}, P:=\sigma(Q)$ is a smooth point of $D_{k}$, and $\sigma$ sends biholomorphically a smooth analytic disc transversal to the divisor $E_{k}$ at $Q$ onto a transversal disc to $D_{k}$ at $P$. As the matrix $M_{\sigma}$ is upper triangular, we obtain $\sigma_{*}^{\prime}\left(H_{i}^{\prime}\right) \subseteq H_{i}(i=1, \ldots, n)$ and furthermore, $\sigma_{*}^{\prime}\left(\alpha_{E_{k}}\right) \sim \alpha_{D_{k}} \bmod H_{k-1}$. As $\sigma_{*}^{\prime}\left(H_{k-1}^{\prime}\right)=H_{k-1}$, it follows that also $\sigma_{*}^{\prime}\left(H_{k}^{\prime}\right)=H_{k}$, therefore $\sigma_{*}^{\prime}\left(H^{\prime}\right)=H$, as desired. 
1.2. Acyclic varieties. Recall that the acyclicity of a topological space $X$ means that its reduced homology vanishes: $\widetilde{H}_{*}(X):=\widetilde{H}_{*}(X ; \mathbb{Z})=0$. The following proposition is an immediate corollary of the above results.

Proposition 1.5. Assume that the affine varieties $X$ and $Y$ as in 1.1 above are smooth and acyclic. Then

(a) $n=n^{\prime}$ and the multiplicity matrix $M_{\sigma}$ is unimodular.

If moreover, $M_{\sigma}$ is an upper triangular unipotent matrix, then

(b) $\sigma_{*}: \pi_{1}(X) \rightarrow \pi_{1}(Y)$ is an isomorphism.

(c) $X$ is contractible if and only if $Y$ is also.

(d) In the latter case the varieties $X$ and $Y$ are both diffeomorphic to the affine space $\mathbb{R}^{2 m}$ provided that $m:=\operatorname{dim}_{\mathbb{C}} Y \geq 3$.

Proof. By [10, 1.18-1.20] (see also [13, 3.2]) for any smooth acyclic affine variety $Z$ the algebra $\mathbb{C}[Z]$ is UFD and its invertible elements are constants. Thus (a) follows from [1.3. (b) directly follows from 1.4 In virtue of the Hurewicz and Whitehead theorems [9], Ch.2, §11.5, §14.2], an acyclic manifold is contractible if and only if it is simply connected. Hence (c) follows from (b). In turn, (d) follows from the Dimca-Ramanujam theorem [4, 43].

1.6. In section 3.2 we will apply the following corollary (see 1.7 below) of Miyanishi's characterization of $\mathbb{C}^{3}[28,29]$. On the other hand, this corollary also follows from [35] and [20, as stated in [20] Cor. 0.2]. Moreover, by [14, L. III] it would be enough to suppose in 1.7 that only general (rather than all) fibers of $x$ were isomorphic to $\mathbb{C}^{2}$.

Theorem 1.7. Let $X$ be a smooth acyclic affine 3 -fold. Then $X \simeq \mathbb{C}^{3}$ if and only if there exists a regular function $x \in \mathbb{C}[X]$ with all fibers isomorphic to $\mathbb{C}^{2}$.

1.8. It is known [19] Thm.1.1] that any birational morphism $\sigma: X \rightarrow Y$ of affine varieties is an affine modification. The divisor $D \subseteq Y$ of modification and the exceptional divisor $E \subseteq X$ of $\sigma$ can be defined as minimal reduced divisors such that the restriction $\left.\sigma\right|_{X \backslash E}: X \backslash E \rightarrow Y \backslash D$ is an isomorphism. In the next proposition and its corollary we provide conditions (more general than those in [19, Thm. 3.1]) which guarantee preservation of the homology group under modification. (Note that the divisors $\hat{E}$ and $\hat{D}$ below do not need to satisfy the assumption of minimality.)

Proposition 1.9. Given affine varieties $\hat{X}, \hat{Y}$ and decompositions

$$
\hat{X}=(\hat{X} \backslash \hat{E}) \cup \hat{E} \quad \text { and } \quad \hat{Y}=(\hat{Y} \backslash \hat{D}) \cup \hat{D},
$$

where $\hat{E} \subseteq \hat{X}$ and $\hat{D} \subseteq \hat{Y}$ are reduced divisors, let $\sigma: \hat{X} \rightarrow \hat{Y}$ be a birational morphism which respects these decompositions. Suppose that the following hold:

(i) $\hat{E}, \hat{D}$ are topological manifolds, $\hat{E} \subseteq \operatorname{reg} \hat{X}, \hat{D} \subseteq \operatorname{reg} \hat{Y}$, and $\sigma^{*}(\hat{D})=\hat{E}$.

(ii) The induced homomorphisms

$$
\left(\left.\sigma\right|_{\hat{E}}\right)_{*}: H_{*}(\hat{E}) \rightarrow H_{*}(\hat{D}) \quad \text { and } \quad\left(\left.\sigma\right|_{\hat{X} \backslash \hat{E}}\right)_{*}: H_{*}(\hat{X} \backslash \hat{E}) \rightarrow H_{*}(\hat{Y} \backslash \hat{D})
$$

are isomorphisms.

Then $\sigma_{*}: H_{*}(\hat{X}) \rightarrow H_{*}(\hat{Y})$ is an isomorphism as well; in particular, $\hat{X}$ is acyclic if and only if $\hat{Y}$ is also. 
Proof. We apply the Thom isomorphism [6, 7.15] to the pairs $(\hat{X}, \hat{E})$, resp., $(\hat{Y}, \hat{D})$ (notice that locally near $\hat{E}$, resp., $\hat{D}$, these are pairs of topological manifolds). As $\sigma^{*}(\hat{D})=\hat{E}$ and $\left(\left.\sigma\right|_{\hat{E}}\right)_{*}: H_{0}(\hat{E}) \stackrel{\cong}{\longrightarrow} H_{0}(\hat{D}), \sigma$ maps the irreducible components of $\hat{E}$ into those of $\hat{D}$ providing a one-to-one correspondence, and (as in 1.2) sends their transverse classes [6, Ch. VIII] to the corresponding transverse classes. By functoriality of the cap-product [6] VII.12.6] for every $i \geq 0$ the following diagram is commutative:

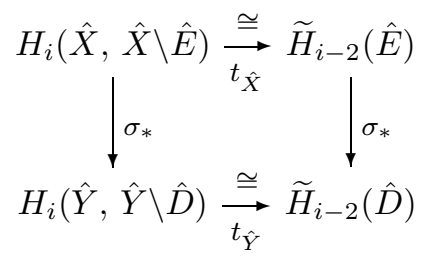

(where $t$ stands for the Thom isomorphism, and the homology groups in negative dimensions are zero). This allows us to replace the relative homology groups in the exact sequences of pairs as to obtain the following commutative diagram:

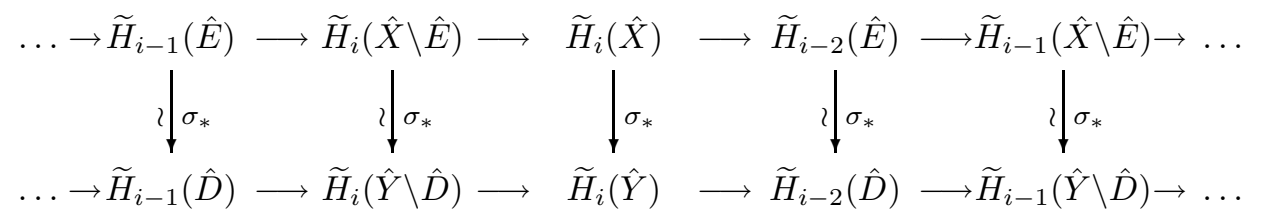

By (ii) the four vertical arrows (as shown at the diagram) are isomorphisms, hence by the 5-lemma, the middle one is also, as stated.

Actually, the divisors $E$ and $D$ we deal with in section 2 below are not always topological manifolds. However, in our setting we can apply 1.9 by decomposing further as follows.

Corollary 1.10. The same conclusion as in 1.9 holds if (instead of (i), (ii)) we assume that there are decompositions $\hat{E}=E^{\prime}+E^{\prime \prime}$ and $\hat{D}=D^{\prime}+D^{\prime \prime}$ satisfying the following conditions:

(i') $E^{\prime} \backslash E^{\prime \prime}, D^{\prime} \backslash D^{\prime \prime}, E^{\prime \prime}$ and $D^{\prime \prime}$ are topological manifolds, $\hat{E} \subseteq \operatorname{reg} \hat{X}, \hat{D} \subseteq$ $\operatorname{reg} \hat{Y}$, and $\sigma^{*}\left(D^{\prime} \backslash D^{\prime \prime}\right)=E^{\prime} \backslash E^{\prime \prime}, \sigma^{*}\left(D^{\prime \prime}\right)=E^{\prime \prime}$.

(ii') The induced homomorphisms $\left(\sigma_{\hat{X} \backslash \hat{E}}\right)_{*}: H_{*}(\hat{X} \backslash \hat{E}) \rightarrow H_{*}(\hat{Y} \backslash \hat{D})$,

$$
\left(\left.\sigma\right|_{E^{\prime} \backslash E^{\prime \prime}}\right)_{*}: H_{*}\left(E^{\prime} \backslash E^{\prime \prime}\right) \rightarrow H_{*}\left(D^{\prime} \backslash D^{\prime \prime}\right) \quad \text { and } \quad\left(\left.\sigma\right|_{E^{\prime \prime}}\right)_{*}: H_{*}\left(E^{\prime \prime}\right) \rightarrow H_{*}\left(D^{\prime \prime}\right)
$$

are isomorphisms.

Proof. Indeed, 1.9 implies that (under our assumptions)

$$
\left(\left.\sigma\right|_{\hat{X} \backslash E^{\prime \prime}}\right)_{*}: H_{*}\left(\hat{X} \backslash E^{\prime \prime}\right) \rightarrow H_{*}\left(\hat{Y} \backslash D^{\prime \prime}\right)
$$

is an isomorphism. Now (with $\hat{E}$ and $\hat{D}$ in 1.9 replaced by $E^{\prime \prime}$, resp., $D^{\prime \prime}$ ), 1.9 implies that $\sigma_{*}: H_{*}(\hat{X}) \rightarrow H_{*}(\hat{Y})$ is an isomorphism as well. 
1.11. Recall [27, Ch. 5, §5.3], [11, Ch. 2, §8.1]1 that a simplicial polyhedron $P$ is called a homology $n$-manifold if for any point $p \in P$ we have $H_{*}(P, P \backslash\{p\}) \cong$ $\widetilde{H}_{*}\left(S^{n}\right)$ 2

Proposition 1.12. Let $X$ be a smooth acyclic affine variety of complex dimension $n$. Then the one-point compactification $\dot{X}$ of $X$ is a homology $2 n$-manifold which is a homology $2 n$-sphere: $H_{*}(\dot{X}) \cong H_{*}\left(S^{2 n}\right)$. In particular, the Alexander duality holds for $\dot{X}$.

Proof. Notice first that $X$ is diffeomorphic to the interior of a compact manifold, say, $X_{R}$, with boundary $\partial X_{R}$ (indeed, one can take for $X_{R}$ the intersection of $X$ with a ball of a large enough radius $R$ in an affine space $\left.\mathbb{C}^{N} \supseteq X\right)$. Hence $\dot{X} \simeq X_{R} / \partial X_{R} \simeq X_{R} \cup_{\partial X_{R}} C\left(\partial X_{R}\right)$ (with $C Y$ denoting the cone over $Y$ ), and any triangulation of $X_{R}$ naturally extends to those of $\dot{X}$.

By the Poincaré-Lefschetz duality for a manifold with boundary [27 5.4.13],

$$
H^{2 n-i}\left(X_{R}\right) \cong H_{i}\left(X_{R}, \partial X_{R}\right) \cong \widetilde{H}_{i}\left(X_{R} / \partial X_{R}\right) \cong \widetilde{H}_{i}(\dot{X}),
$$

hence $\dot{X}$ is a homology $2 n$-sphere. Using the acyclicity of $X_{R}$ and of $C\left(\partial X_{R}\right)$ and applying the Mayer-Vietoris sequence to the decomposition $\dot{X}=X_{R} \cup_{\partial X_{R}} C\left(\partial X_{R}\right)$, we see that $H_{i}(\dot{X}) \cong H_{i-1}\left(\partial X_{R}\right)$. Thus the smooth manifold $\partial X_{R}$ is a homology $(2 n-1)$-sphere.

Clearly, $H_{*}(\dot{X}, \dot{X} \backslash\{x\}) \cong \widetilde{H}_{*}\left(S^{2 n}\right)$ for any point $x \in X=\dot{X} \backslash\{\infty\}$. For the vertex $x=\infty$ of the cone $C\left(\partial X_{R}\right)$ and for any $i \in \mathbb{N}$, by excision and from the exact homology sequence of a pair we obtain

$$
\begin{aligned}
& H_{i}(\dot{X}, \dot{X} \backslash\{\infty\}) \cong H_{i}\left(C\left(\partial X_{R}\right), C\left(\partial X_{R}\right) \backslash\{\infty\}\right) \\
& \quad \cong \widetilde{H}_{i-1}\left(\partial X_{R}\right) \cong \widetilde{H}_{i-1}\left(S^{2 n-1}\right) \cong \widetilde{H}_{i}\left(S^{2 n}\right) .
\end{aligned}
$$

Thereby $\dot{X}$ is a homology manifold and is a homology $2 n$-sphere. For any point $x \in \dot{X}$, from the exact homology sequence of the pair $(\dot{X}, \dot{X} \backslash\{x\})$ it follows that $\dot{X} \backslash\{x\}$ is acyclic. Now the proof of the Alexander duality for the usual sphere [27. 5.3.19] goes mutatis mutandis for $\dot{X}$ (cf. [3, Thm. 6.4], [40 p. 176]).

1.3. Digest on Makar-Limanov and Derksen invariants. These invariants (introduced in [23, 15, 5]) allow in certain cases to distinguish space-like affine varieties from the affine spaces. For this purpose, we use them in subsection 3.1 (to establish (3.6). Let us first recall the following notions and facts.

1.13. Locally nilpotent derivations. Let $A$ be an affine domain over $\mathbb{C}$. A derivation $\partial \in \operatorname{Der} A$ of $A$ is called locally nilpotent ( $L N D$ for short) if for each $a \in A$ there exists $n=n(a, \partial) \in \mathbb{N}$ such that $\partial^{(n)}(a)=0$; the set of all non-zero locally nilpotent derivations of the algebra $A$ is denoted by $\operatorname{LND}(A)$. Given $\partial \in \operatorname{LND}(A)$, the function $\operatorname{deg}_{\partial}(a):=\min \{n(a, \partial)-1\}$ is a degree function on $A$. The kernel $A^{\partial}=\operatorname{ker} \partial$ of $\partial$ is a $\partial$-invariant subalgebra of $A$; its elements are called $\partial$-constants. For the proof of the following lemma see, e.g., [23, 24, 15, 5], [43, §7] or [14, 5.1(6)].

\footnotetext{
${ }^{1}$ We are grateful to L. Guillou for useful discussions on homology manifolds and references.

${ }^{2}$ Or equivalently, $H_{*}(l k(p)) \cong H_{*}\left(S^{n-1}\right)$, where $l k(p)$ denotes the link of $p$ in $P$, or else, for any $q$-simplex $\sigma$ in $P, H_{*}(l k(\sigma)) \cong H_{*}\left(S^{n-q-1}\right)$.
} 
Lemma 1.14. The following statements hold:

(a) tr.deg $\left[A: A^{\partial}\right]=1$.

(b) The subalgebra $A^{\partial}$ is algebraically closed.

(c) It is factorially closed, i.e., $u v \in A^{\partial} \backslash\{0\} \Longrightarrow u, v \in A^{\partial}$.

(d) If $u^{k}+v^{l} \in A^{\partial} \backslash\{0\}$ for some $k, l \geq 2$, then $u, v \in A^{\partial}$.

Moreover, $p(u, v) \in A^{\partial} \backslash \mathbb{C} \Longrightarrow u, v \in A^{\partial}$ for any polynomial $p \in \mathbb{C}^{[2]}$ with general fibers being irreducible and non-isomorphic to $\mathbb{C}$.

1.15. Invariants. The Makar-Limanov invariant of the algebra $A$ is the subalgebra

$$
\operatorname{ML}(A)=\bigcap_{\partial \in \operatorname{LND}(A)} A^{\partial} \subset A,
$$

whereas the Derksen invariant

$$
\operatorname{Dk}(A)=\mathbb{C}\left[\bigcup_{\partial \in \operatorname{LND}(A)} A^{\partial}\right] \subset A
$$

is the subalgebra of $A$ generated by the $\partial$-constants of all locally nilpotent derivations on $A$. If $\operatorname{ML}(A)=\mathbb{C}$ (resp., $\operatorname{Dk}(A)=A$ ), then we say that the corresponding invariant is trivial. This is, indeed, the case for a polynomial algebra $A=\mathbb{C}^{[n]}(n \in \mathbb{N})$.

1.16. Specializations. Let $X$ be an affine variety, and set $A=\mathbb{C}[X]$. To study the locally nilpotent derivations on $A$, it is possible to proceed by induction on the dimension of $X$. Namely, let $\partial \in \operatorname{LND}(A)$, and let $u \in \operatorname{ker} \partial$ be non-zero. As $\partial(u-c)=0 \forall c \in \mathbb{C}$, the principal ideal $(u-c)$ of the algebra $A$ is invariant under $\partial$, and so $\partial$ descends to the quotient $B_{c}=A /(u-c)=\mathbb{C}\left[S_{c}\right]$, where $S_{c}=u^{-1}(c)$ is a fiber of $u$. For a general $c \in \mathbb{C}$, this specialization $\partial_{c}$ is a non-zero locally nilpotent derivation of the algebra $B_{c}$ (see 1.17 below). Clearly, the restriction to $S_{c}$ of any $\partial$-constant $v \in \operatorname{ker} \partial$ is a $\partial_{c}$-constant.

1.17. $\mathbb{C}_{+}$-actions. Otherwise, the above specialization can be described via the natural correspondence between the locally nilpotent derivations of the algebra $A$ and the regular actions of the additive group $\mathbb{C}_{+}$on the variety $X=\operatorname{spec} A$ (e.g., see [31, 43]). Indeed, the subalgebra ker $\partial$ coincides with the algebra of invariants $A^{\varphi}$ of the associated $\mathbb{C}_{+}$-action $\varphi=\varphi_{\partial}$. If $u$ is a $\varphi$-invariant, then clearly, the $\mathbb{C}_{+-}$ action $\left.\varphi\right|_{S_{c}}$ on a fiber $S_{c}=\operatorname{spec} B_{c}$ of $u$ is associated with the above specialization $\partial_{c}$. Hence $\partial_{c} \in \operatorname{LND}\left(B_{c}\right)$ if and only if the $\mathbb{C}_{+}$-action $\left.\varphi\right|_{S_{c}}$ is non-trivial.

1.18. Jacobian derivations [15, [16]. For an $n$-tuple of polynomials $p_{1}, \ldots, p_{n-1}, q \in$ $\mathbb{C}^{[n]}$, the Jacobian

$$
\partial(q):=\operatorname{jac}\left(p_{1}, \ldots, p_{n-1}, q\right)=\frac{\partial\left(p_{1}, \ldots, p_{n-1}, q\right)}{\partial\left(x_{1}, \ldots, x_{n}\right)}
$$

(regarded as a function of $q$, whereas the polynomials $p_{1}, \ldots, p_{n-1}$ are fixed) gives a derivation on the polynomial algebra $\mathbb{C}^{[n]}$. This derivation is non-zero provided that the polynomials $p_{1}, \ldots, p_{n-1}$ are algebraically independent. For $p:=p_{1}$, the principal ideal $(p) \subseteq \mathbb{C}^{[n]}$ is invariant under $\partial$, hence $\partial$ descends to a derivation of the quotient algebra $A:=\mathbb{C}^{[n]} /(p)=\mathbb{C}[X]$, where $X:=p^{*}(0) \subset \mathbb{C}^{n}$ 目 Denote

\footnotetext{
${ }^{3}$ To simplify the exposition we suppose that $X$ is a hypersurface, whereas the results of [16] hold in a more general setting.
} 
by $\operatorname{JLND}(A)$ the set of all locally nilpotent Jacobian derivations of the algebra $A$. Two derivations $\partial, \partial^{\prime} \in \operatorname{Der}(A)$ are called equivalent if $A^{\partial}=A^{\partial^{\prime}}$. Actually, two derivations are equivalent iff they generate the same degree function [16]: $\operatorname{deg}_{\partial}=$ $\operatorname{deg}_{\partial^{\prime}}$. We have the following theorem.

Theorem 1.19 (15 16]). If $p \in \mathbb{C}^{[n]}$ is a non-constant irreducible polynomial, then every locally nilpotent derivation $\partial \in \operatorname{LND}(A)$ (where as above, $A=\mathbb{C}^{[n]} /(p)$ ) is equivalent to a Jacobian locally nilpotent derivation $\partial^{\prime} \in \operatorname{JLND}(A)$. Henceforth, we have

$$
\operatorname{ML}(A)=\bigcap_{\partial \in \operatorname{JLND}(A)} A^{\partial} \quad \text { and } \quad \operatorname{Dk}(A)=\mathbb{C}\left[\bigcup_{\partial \in \operatorname{JLND}(A)} A^{\partial}\right] .
$$

1.20. Weight degree functions and the associated graded algebras (see, e.g., [15, 16], 43]). A weight degree function on the algebra $A=\mathbb{C}[X]=\mathbb{C}^{[n]} /(p)$ is defined by assigning weights $d_{i}=d\left(x_{i}\right) \in \mathbb{R}$ to the variables $x_{1}, \ldots, x_{n} \in \mathbb{C}^{[n]}$ and letting for $a \in A$

Here as usual

$$
d_{A}(a):=\inf \left\{d(q)\left|q \in \mathbb{C}^{[n]}, q\right|_{X}=a\right\} .
$$

$$
d(q)=\max \{d(m) \mid m \in M(q)\}
$$

with $M(q)$ denoting the set of the monomials $m=c_{m} \prod_{i=1}^{n} x_{i}^{\alpha_{i}}$ of $q$, where $d(m):=$ $\sum_{i=1}^{n} \alpha_{i} d_{i}$. The weight degree function $d_{A}: A \rightarrow \mathbb{R} \cup\{-\infty\}$ defines an ascending filtration $\left\{A_{t}\right\}_{t \in \mathbb{R}}$ of the algebra $A$ with $A_{t}:=\left\{a \in A \mid d_{A}(a) \leq t\right\}$. We also let $A_{t}^{\prime}:=\left\{a \in A \mid d_{A}(a)<t\right\} \subset A_{t}$, and we consider the associated graded algebra

$$
\hat{A}=\bigoplus_{t \in \mathbb{R}} A_{t} / A_{t}^{\prime}
$$

(actually, the set of non-zero homogeneous components $A_{t} / A_{t}^{\prime}$ of the algebra $\hat{A}$ is at most countable).

1.21. Associated homogeneous derivations. For a polynomial $q \in \mathbb{C}^{[n]}$, we consider its $d$-principal part (in other words, the principal d-quasihomogeneous part) $\hat{q}:=$ $\sum_{m \in M(q), d(m)=d(q)} m$. For an element $a \in A$, we let $\hat{a}$ be its image in the graded algebra $\hat{A}$ (clearly, $\hat{a} \in A_{t} / A_{t}^{\prime}$ with $t=d_{A}(a)$ ). Notice that $\hat{a}=\left.\hat{q}\right|_{\hat{X}}$ for $\hat{X}=\hat{p}^{-1}(0)$ and a polynomial $q \in \mathbb{C}^{[n]}$ such that $\left.q\right|_{X}=a$ and $d(q)=d_{A}(a)$; the latter equality holds if and only if $\left.\hat{q}\right|_{\hat{X}} \neq 0$.

If $\partial \in \operatorname{Der}(A)$ is a derivation, then the degree

$$
d_{A}(\partial):=\inf \left\{d_{A}(a)-d_{A}(\partial a) \mid a \in A\right\}
$$

is finite [16]. Letting

$$
\hat{\partial} \hat{a}:=\widehat{\partial a} \quad \text { if } \quad d_{A}(a)-d_{A}(\partial a)=d_{A}(\partial) \quad \text { and } \quad \hat{\partial} \hat{a}=0 \quad \text { otherwise, }
$$

and extending $\hat{\partial}$ in a natural way to a homogeneous derivation of the graded algebra $\hat{A}$, we obtain a correspondence $\operatorname{Der}(A) \rightarrow \operatorname{Der}_{\mathrm{gr}}(\hat{A})$. It has the following properties.

Theorem $1.22([15,16])$. Let $p \in \mathbb{C}^{[n]}$ be a polynomial with a non-constant, irreducible d-principal part $\hat{p}$. Set $\hat{X}=\hat{p}^{-1}(0) \subset \mathbb{C}^{n}$. Then the following hold:

(a) $\hat{A} \simeq \mathbb{C}[\hat{X}]$. 
(b) If $\partial \in \operatorname{LND}(A)$, then $\hat{\partial} \in \operatorname{LND}(\hat{A})$, and for any $a \in A^{\partial}, \hat{a} \in \hat{A}^{\hat{\partial}}$.

(c) 4 For any non-zero Jacobian derivation

$$
\partial=\frac{\partial\left(p, p_{2}, \ldots, p_{n-1}, *\right)}{\partial\left(x_{1}, \ldots, x_{n}\right)}
$$

of the algebra $A=\mathbb{C}[X]$ there exists an equivalent one

$$
\partial^{\prime}=\frac{\partial\left(p, p_{2}^{\prime}, \ldots, p_{n-1}^{\prime}, *\right)}{\partial\left(x_{1}, \ldots, x_{n}\right)}
$$

such that the d-principal parts $\hat{p}, \hat{p}_{2}^{\prime}, \ldots, \hat{p}_{n-1}^{\prime}$ are algebraically independent.

(d) If the latter condition holds, then the associated derivation $\hat{\partial}^{\prime} \in \operatorname{Der}(\hat{A})$ of the associated graded algebra $\hat{A}$ is also a Jacobian one, namely,

$$
\hat{\partial}^{\prime}=\frac{\partial\left(\hat{p},{\hat{p^{\prime}}}_{2}, \ldots,{\hat{p^{\prime}}}_{n-1}, *\right)}{\partial\left(x_{1}, \ldots, x_{n}\right)} .
$$

1.23. Graded invariants. For a graded algebra $\hat{A}$, we denote by $\operatorname{Dk}_{\mathrm{gr}}(\hat{A})$ the following 'graded' version of the Derksen invariant:

$$
\operatorname{Dk}_{\mathrm{gr}}(\hat{A})=\mathbb{C}\left[\bigcup_{\partial \in \operatorname{LND}_{\mathrm{gr}}(\hat{A})} \hat{A}^{\partial}\right] .
$$

The way we use the Derksen invariant (similar to that of [5] 15, 23, 43]) in the next section is based on the next simple lemma.

Lemma 1.24. Given an irreducible hypersurface $X \subseteq \mathbb{C}^{n}$, suppose that the algebra $A=\mathbb{C}[X]$ is equipped with a weight degree function $d_{A}$ such that the hypersurface $\hat{X} \subset \mathbb{C}^{n}$ is also irreducible. If

$$
\operatorname{Dk}_{\mathrm{gr}}(\hat{A}) \subset \hat{A}_{\leq 0}:=\bigoplus_{t \leq 0} A_{t} / A_{t}^{\prime} \subset \hat{A},
$$

then

$$
\operatorname{Dk}(A) \subset A_{0}=\left\{a \in A \mid d_{A}(a) \leq 0\right\} .
$$

Henceforth, $X \not \mathbb{C}^{n-1}$ unless $A=A_{0}$.

Proof. By 1.22 (a),(b) for every $\partial \in \operatorname{LND}(A)$ and for every $\partial$-constant $a \in A^{\partial}$, we have $\hat{a} \in \operatorname{ker} \hat{\partial}$, where $\hat{\partial} \in \operatorname{LND}_{\mathrm{gr}}(\hat{A})$, hence $\hat{a} \in \hat{A}_{\leq 0}$. Therefore, $d_{A}(a) \leq 0$, i.e., $a \in A_{0}$, as stated.

\subsection{Variables in polynomial rings.}

1.25. For a commutative $\operatorname{ring} B$, we let $B^{[n]}=B\left[y_{1}, \ldots, y_{n}\right]$. A polynomial $p \in B^{[n]}$ is called a $B$-variable (or simply a variable if no ambiguity occurs) if it is a coordinate $\left(p=p_{1}\right)$ of an automorphism $\beta=\left(p_{1}, p_{2}, \ldots, p_{n}\right) \in \operatorname{Aut}_{B} B^{[n]}$ (thus $\left.\left.\beta\right|_{B}=\operatorname{id}_{B}\right)$. The set of all $B$-variables of $B^{[n]}$ is denoted as $\operatorname{Var}_{B} B^{[n]}$. For $B=\mathbb{C}\left[x_{1}, \ldots, x_{n}\right]$, a $B$-automorphism is called in brief an $\left(x_{1}, \ldots, x_{n}\right)$-automorphism, and a $B$-variable is also called an $\left(x_{1}, \ldots, x_{n}\right)$-variable.

\footnotetext{
${ }^{4} \mathrm{~A}$ similar fact remains true for any affine domain [25].
} 
Remark 1.26. It is easily seen that a polynomial $p \in \mathbb{C}^{[n]}$ is an $\left(x_{1}, \ldots, x_{k}\right)$-variable (where $0 \leq k \leq n-1$ ) if and only if $X:=p^{-1}(0) \simeq \mathbb{C}^{n-1}$ and there exists $\partial \in \operatorname{LND}\left(\mathbb{C}^{[n]}\right)$ with $x_{1}, \ldots, x_{k} \in \operatorname{ker} \partial$ such that $\partial p=1$ (i.e., $p$ is a slice of $\partial$ ). Indeed, $\partial p=1$ implies that $X$ can be identified with the orbit space $\mathbb{C}^{n} / \varphi_{\partial}=$ $\operatorname{spec}\left(\mathbb{C}^{[n]}\right)^{\varphi_{\partial}}=\operatorname{spec}(\operatorname{ker} \partial)$ of the associated $\mathbb{C}_{+}$-action $\varphi_{\partial}$ on $\mathbb{C}^{n}$ (see 1.17), and so $\mathbb{C}^{[n]}=(\operatorname{ker} \partial)[p] \cong \mathbb{C}^{[n-1]}[p]$.

Notice that as $\mathbb{C}^{n} \simeq X \times \mathbb{C}$, the assumption $X \simeq \mathbb{C}^{n-1}$ above is superfluous provided that the Zariski Cancellation Conjecture holds.

1.27. For a polynomial $q=q(x, y, z, \ldots) \in \mathbb{C}^{[n]}$, we denote by $q_{\lambda}(\lambda \in \mathbb{C})$ the specialization $q(\lambda, y, z, \ldots) \in \mathbb{C}^{[n-1]}$.

We say that a polynomial $p\left(x, y_{1}, \ldots, y_{n}\right) \in \mathbb{C}[x]\left[y_{1}, \ldots, y_{n}\right]$ is an $x$-residual variable if for every $\lambda \in \mathbb{C}$, the specialization $p_{\lambda}$ is a variable of $\mathbb{C}\left[y_{1}, \ldots, y_{n}\right]$. It is easily seen that any $x$-variable is an $x$-residual variable.

1.28. Let $f \in B$. An element $b \in B$ is said to be invertible (resp., nilpotent) $\bmod f$ if its image $[b] \in B /(f)$ is also. Thus $b$ is nilpotent $\bmod f$ if and only if $b \in \operatorname{rad}(f)$. Observe that if $B$ is UFD and $f=\prod_{i=1}^{n} f_{i}^{a_{i}}$ is a canonical factorization, then $b \in \operatorname{rad}(f) \Leftrightarrow b \in\left(f^{\text {red }}\right)$, where $f^{\text {red }}:=\prod_{i=1}^{n} f_{i} \in B$.

To detect variables in polynomial rings, the following results will be useful. The statement (a) below is due to P. Russell [32, Prop. 2.2], and is reproved in [39] based on (b) (see [39. Prop. 1.4], or also [8]).

Proposition 1.29. For a commutative ring B, the following hold:

(a) For any $f, b_{0}, b_{1} \in B$ and $q \in B[z]$ with $b_{1}$ invertible $\bmod f$ and $q$ nilpotent $\bmod f$, we have $p:=f u+b_{0}+b_{1} z+q(z) \in \operatorname{Var}_{B} B[z, u]$.

(b) For $f \in B$, we let $B_{f}:=B /(f)$, and we denote by $\rho: B \rightarrow B_{f}$ the canonical surjection. If $p \in \operatorname{Var}_{B} B[z, u]$ is such that $\rho(p(z, 0)) \in \operatorname{Var}_{B_{f}} B_{f}[z]$, then also $p_{f}(z, u):=p(z, f u) \in \operatorname{Var}_{B} B[z, u]$.

(c) Let $B=\mathbb{C}[x]$ and $f \in B$. If $p \in \operatorname{Var}_{B} B[y, z, u]$ is such that for every root $x_{0}$ of $f$, the specialization $p_{x_{0}}(y, z, 0)$ is a variable of $\mathbb{C}[y, z] 5$ then also $p_{f}(y, z, u):=p(y, z, f u) \in \operatorname{Var}_{B} B[y, z, u]$.

Proof of (c). It suffices to prove (c) for $f=x$ and then conclude by induction on the degree of $f$. Let $\gamma \in \operatorname{Aut}_{B} B[y, z, u]$ be such that $\gamma(y)=p(x, y, z, u)$. Denote $\gamma_{0}$ as the specialization of $\gamma$ at $x=0$. Clearly, $\gamma_{0}(y)=p(0, y, z, u)$ is a variable of $\mathbb{C}[y, z, u]$; in particular,

$$
\mathbb{C}[y, z, u] /(p(0, y, z, u)) \simeq \mathbb{C}^{[2]}
$$

If $\phi$ denotes this isomorphism, then

$$
\mathbb{C}[y, z, u] /(p(0, y, z, u), u) \simeq \mathbb{C}^{[2]} /(\phi(u)) .
$$

By our assumption, $p(0, y, z, 0)$ is a variable of $\mathbb{C}[y, z]$, and sd

$$
\mathbb{C}[y, z, u] /(p(0, y, z, u), u) \simeq \mathbb{C}[y, z] /(p(0, y, z, 0)) \simeq \mathbb{C}^{[1]} .
$$

\footnotetext{
${ }^{5}$ Or equivalently, $\rho(p(y, z, 0)) \in \operatorname{Var}_{B_{f}} B_{f}[y, z]$.

${ }^{6}$ Hereafter by $(f, g)$ we mean the ideal generated by $f$ and $g$.
} 
Hence by the Abhyankar-Moh-Suzuki Theorem $\left([1,38) \phi(u)\right.$ is a variable in $\mathbb{C}^{[2]}$. Therefore we may assume that $\phi(u)=u$, i.e., there is a $\mathbb{C}[u]$-isomorphism

$$
\mathbb{C}[u][y, z] /(p(0, y, z, u)) \simeq \mathbb{C}[u]^{[1]} .
$$

By the Abhyankar-Moh-Suzuki theorem as generalized by Russell and Sathaye [33. Thm. 2.6.2], $p(0, y, z, u)$ is a $\mathbb{C}[u]$-variable of $\mathbb{C}[u][y, z]$. Let $\alpha_{0}$ be the corresponding $\mathbb{C}[u]$-automorphism of $\mathbb{C}[u][y, z]$ such that $\alpha_{0}(y)=p(0, y, z, u)$, and denote by $\bar{\gamma}$ the composition

$$
\bar{\gamma}:=\gamma \gamma_{0}^{-1} \alpha_{0} \in \operatorname{Aut}_{B} B[y, z, u] .
$$

Then we get

$$
\bar{\gamma}(y)=\gamma \gamma_{0}^{-1} \alpha_{0}(y)=\gamma \gamma_{0}^{-1}(p(0, y, z, u))=\gamma(y)=p(x, y, z, u)
$$

and

$$
\bar{\gamma}(u)=\gamma \gamma_{0}^{-1} \alpha_{0}(u)=\gamma \gamma_{0}^{-1}(u) \equiv u \bmod x .
$$

If $\sigma_{u}$ is the $(x, y, z)$-automorphism of $\mathbb{C}(x)[y, z][u]$ defined by $\sigma_{u}(u)=x u$, then the composition $\sigma_{u} \bar{\gamma} \sigma_{u}^{-1}$ (which a priori is an $x$-automorphism of $\mathbb{C}(x)[y, z, u]$ ) actually is a $B$-automorphism of $B[y, z, u]$. Thus $\sigma_{u} \bar{\gamma} \sigma_{u}^{-1}(y)=p(x, y, z, x u)$ is an $x$-variable of $\mathbb{C}[x][y, z, u]$.

Remark 1.30. We would like to use this opportunity to indicate a flaw in the proof of Theorem 7.2 in [19] (which generalizes the Sathaye theorem mentioned in the introduction). Namely, proving Proposition 7.1 in [19] and carrying induction by the multiplicity of a root $x=0$ of the polynomial $p$, it was forgotten to extend it over all the roots. Instead, one can apply [1.29)(a) (cf. also [39, Thm. 3.6]) which fixes the flaw and simplifies the proof considerably.

Corollary 1.31. Let $p \in \mathbb{C}[x][y, z, u]$ be an $x$-variable.

(a) If for $q(x) \in \mathbb{C}[x], p_{q}:=p(x, y, z, q(x) u)$ is an $x$-residual variable, then it actually is an $x$-variable.

(b) Consequently, $p(x, y, z, q(x) u)$ is an $x$-variable if and only if $p\left(x, y, z, q_{\text {red }}(x) u\right)$ is also.

Proof. (a) As $p_{q}$ is an $x$-residual variable, for every root $x_{0}$ of $q, p_{x_{0}, 0}:=p\left(x_{0}, y, z, 0\right)$ is a variable of $\mathbb{C}[y, z, u]$. In particular, $\mathbb{C}[y, z, u] /\left(p_{x_{0}, 0}\right)=\mathbb{C}[y, z] /\left(p_{x_{0}, 0}\right) \otimes \mathbb{C}[u] \cong$ $\mathbb{C}^{[2]}$. It follows that $\mathbb{C}[y, z] /\left(p_{x_{0}, 0}\right) \cong \mathbb{C}^{[1]}$, and then by the Abhyankar-Moh-Suzuki Theorem $p_{x_{0}, 0}$ is a variable of $\mathbb{C}[y, z]$. It follows from 1.29 (c) that $p(x, y, z, q(x) u)$ is an $x$-variable, as stated.

The proof of (b) relies on (a) and on the fact that $p(x, y, z, q(x) u)$ and $p\left(x, y, z, q_{\text {red }}(x) u\right)$ are simultaneously $x$-residual variables.

The proof of the following results is inspired by those of [36, Thm. 1.1] and [37. Thm. 1.4].

Proposition 1.32. For any commutative ring B, the following hold:

(a) For an arbitrary pair of polynomials $p, q \in B[y]$ there exists an automorphism $\gamma=\gamma_{p, q} \in \operatorname{Aut}_{B} B[y, v]$ such that

$$
\gamma((y-q(p(y)), v))=(y-p(q(y)), v) .
$$


(b) In particular, for $q(y)=-$ ay with $a \in B$ we have

$$
\gamma((y+a p(y), v))=(y+p(a y), v) .
$$

Moreover, if $a^{k} \mid p(0)$, then there exists an automorphism $\gamma_{k} \in \operatorname{Aut}_{B} B[y, v]$ such that

$$
\gamma_{k}((y+a p(y), v))=\left(y+\frac{p\left(a^{k+1} y\right)}{a^{k}}, v\right) .
$$

Proof. It is not difficult to verify that the desired automorphism $\gamma$ and its inverse $\gamma^{-1}$ can be defined as follows:

$$
\left\{\begin{array} { l } 
{ \gamma ( y ) = v + q ( y ) , } \\
{ \gamma ( v ) = y - p ( v + q ( y ) ) , }
\end{array} \quad \left\{\begin{array}{l}
\gamma^{-1}(y)=v+p(y), \\
\gamma^{-1}(v)=y-q(v+p(y)) .
\end{array}\right.\right.
$$

Iterating $\gamma$ yields an appropriate $\gamma_{k}$ as needed in (b).

Corollary 1.33. (a) For any pair $p, q \in B[y]$ the homomorphism

$$
\phi: B[y] /(y-q(p(y))) \longrightarrow B[y] /(y-p(q(y)))
$$

is an isomorphism.

(b) In particular, for any $a \in B$ and $p \in B[y]$ the homomorphism

$$
\begin{aligned}
\phi: B[y] /(y+a p(y)) & \longrightarrow B[y] /(y+p(a y)) \\
y & \longmapsto a y
\end{aligned}
$$

is an isomorphism. Moreover, if $a^{k} \mid p(0)$ then also

$$
\begin{aligned}
\phi_{k}: B[y] /(y+a p(y)) & \longrightarrow B[y] /\left(y+\frac{p\left(a^{k+1} y\right)}{a^{k}}\right) \\
y & \longmapsto a^{k+1} y
\end{aligned}
$$

is an isomorphism.

Proof. We just apply 1.32 and the obvious isomorphism $B[y] /(f(y)) \simeq$ $B[y, v] /(f(y), v)$.

\section{Simple MODIFICATIONS OF ACYCLIC 3-FOLDS ALONG CYLINDRICAL DIVISORS}

We focus below on a special case of affine modifications called simple birational extensions (see 2.1 below), applied to acyclic affine 3 -folds. Our aim in this section is to give a criterion for as when the acyclicity is preserved under such a modification (cf. [19 Thm. 3.1]). In the special case when the divisor of modification is a cylinder, we obtain in Theorem 2.11 below necessary conditions for preserving the acyclicity. In Theorem 2.27 (cf. also 2.28) we show that these conditions are also sufficient, provided that the given acyclic 3 -fold is a cylinder as well.

\subsection{Simple affine modifications.}

Definition 2.1. A simple birational extension of a domain $A$ (over $\mathbb{C}$ ) is an algebra $A^{\prime}:=A[g / f]$, where $f, g \in A$ are such that the ideal $I=(f, g) \subset A$ is of height 2 (i.e., the center of modification $C=V(I)=D_{f}^{\text {red }} \cap D_{g}^{\text {red }}$ is of codimension 2 in $Y:=\operatorname{spec} A)$. We also call $X:=\operatorname{spec} A^{\prime}$ a simple affine modification of the affine variety $Y$.

In the sequel $A$ is UFD, and the above condition simply means that $f, g \in A$ are coprime. More generally [19] Prop. 1.1], any affine modification can be obtained 
as $A^{\prime}=A\left[g_{1} / f_{1}, \ldots, g_{n} / f_{n}\right]$ with $f_{i}, g_{i} \in A$; here again, if $A$ is UFD, then we may suppose $f_{i}, g_{i}$ is coprime $(i=1, \ldots, n)$.

2.2. Observe that the variety $X=\operatorname{spec} A^{\prime}$ can be realized as the hypersurface in $Y \times \mathbb{C}$ with the equation $f u+g=0$ (where $u$ is a coordinate in $\mathbb{C}$ ), and the blowup morphism is just the first projection: $\sigma=\left.\operatorname{pr}_{1}\right|_{X}: X \rightarrow Y$. The exceptional divisor $E=\sigma^{-1}(C)=\sigma^{-1}(D)=\{f=0\} \subseteq X$ is cylindrical: $E \simeq C \times \mathbb{C}$ (cf. [19, Prop. 1.1]).

We have the following simple but useful lemma.

Lemma 2.3. Using the notation as above, assume that the affine variety $Y:=$ $\operatorname{spec} A$ is smooth. Then the simple affine modification $X=\operatorname{spec} A[g / f]$ is also smooth if and only if

(i) the divisor $D_{g}=g^{*}(0)$ is smooth and reduced at each point of the center $C=D_{f}^{\mathrm{red}} \cap D_{g}^{\mathrm{red}}$, and

(ii) $D_{f}$ and $D_{g}$ meet transversally at those points of $C$ where the divisor $D_{f}$ is also smooth and reduced.

Furthermore, if $X$ is smooth, then $d f=0$ at each singular point of the center $C$.

Proof. The second assertion easily follows from the first one. To prove the first one, let $F=0$ be the equation of the hypersurface $X \subseteq Y \times \mathbb{C}$ with $F:=u f-g \in$ $\mathbb{C}[Y][u]$ being irreducible. As $X \backslash E \simeq Y \backslash D$ is smooth, we should only control the smoothness of $X$ at the points of the exceptional divisor

$$
E=\{f=g=0\}=C \times \mathbb{C} \subseteq Y \times \mathbb{C} .
$$

At a point $Q=(P, u) \in E$, we have

$$
d F=u d f+d g+f d u=u d f+d g=0
$$

if and only if $d g=-u d f$ is proportional to $d f$. Now the assertion easily follows.

2.2. Preserving acyclicity: necessary conditions. In this subsection we adopt the following convention and notation.

Convention 2.4. $\quad$ (i) $X$ and $Y$ denote smooth, acyclic affine 3 -folds 7 such that

(ii) $A^{\prime}=\mathbb{C}[X]$ is a simple birational extension of the algebra $A=\mathbb{C}[Y]$, i.e., $A^{\prime}=A[g / f]$ with coprime elements $f, g \in A$, and

(iii) $D:=D_{f}^{\text {red }} \simeq \Gamma \times \mathbb{C}$ (where $\Gamma$ is an affine curve) is a cylindrical divisor.

2.5. Denote by $\pi: D \rightarrow \Gamma$ the morphism induced by the canonical projection $\Gamma \times \mathbb{C} \rightarrow \Gamma$. By abuse of notation, we equally denote by $\pi$ the restriction $\left.\pi\right|_{C}: C \rightarrow$ $\Gamma$. Thus we have the following commutative diagram:

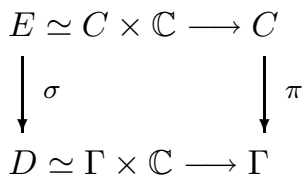

If $C=\bigcup_{i=1}^{n} C_{i}$, resp., $\Gamma=\bigcup_{j=1}^{n} \Gamma_{i}$, is the irreducible decomposition, then the irreducible components of the divisor $E$, resp., $D$, are $E_{i} \simeq C_{i} \times \mathbb{C}$, resp., $D_{j} \simeq$

\footnotetext{
${ }^{7}$ Hence the algebras $A$ and $A^{\prime}$ are UFD's; see the proof of 1.5
} 
$\Gamma_{j} \times \mathbb{C}$. As in 1.1 we denote by $M_{\sigma}=\left(m_{i j}\right)$ the multiplicity matrix of $\sigma$. Recall that by 1.1 1.2 and 2.3. $m_{i j} \geq 1$ if and only if $C_{j} \subset D_{i}^{\text {red }}$, and $m_{i j}=1$ if and only if the surfaces $D_{i}^{\text {red }}, D_{g}^{\text {red }} \subseteq Y$ meet transversally at general points of the curve $C_{j}$.

The terminology in the following definitions comes from the real picture corresponding to our situation.

2.6. Notice that for each $j=1, \ldots, n$ there exists $i \in\{1, \ldots, n\}$ such that $\pi\left(C_{j}\right) \subseteq$ $\Gamma_{i}$, and this index $i=i(j)$ is unique unless $\left.\pi\right|_{C_{j}}=$ const.

An irreducible component $C_{j}$ of the curve $C$ is called vertical if $\pi \mid C_{j}=$ const (i.e. $\operatorname{deg}\left(\left.\pi\right|_{C_{j}}\right)=0$ ) and non-vertical otherwise (thus the vertical components of $C$ are disjoint and each of them is isomorphic to $\mathbb{C}$ ). The uniqueness of the index $i=i(j)$ for a non-vertical component $C_{j}$ and the unimodularity of $M_{\sigma}$ (see 1.5) imply that the $j$-th column of the matrix $M_{\sigma}$ is the $i$-th vector of the standard basis $\left(\bar{e}_{1}, \ldots \bar{e}_{n}\right)$ in $\mathbb{R}^{n}$, and two different non-vertical components $C_{j}$ and $C_{j^{\prime}}$ of $C$ project into two different irreducible components $\Gamma_{i}$, resp., $\Gamma_{i^{\prime}}$, of $\Gamma$. Hence up to reordering, we may assume that $C_{1}, \ldots, C_{k}$ are the non-vertical components of $C$ and $\pi\left(C_{i}\right) \subseteq \Gamma_{i}, i=1, \ldots, k$. Then we have

$$
M_{\sigma}=\left(\begin{array}{c|c}
I_{k} & B \\
--- & --- \\
0 & B^{\prime}
\end{array}\right)
$$

with a unimodular matrix $B^{\prime}$. Consequently, for every $i=1, \ldots, k$, the surfaces $D_{i}^{\text {red }}$ and $D_{g}^{\text {red }}$ meet transversally at general points of the curve $C_{i}$.

2.7. The irreducible components $\Gamma_{1}, \ldots, \Gamma_{k}$ are also called non-vertical, resp., $\Gamma_{k+1}, \ldots, \Gamma_{n}$, are called vertical. Among the non-vertical components $C_{i}$, resp., $\Gamma_{i}$, we distinguish those with $\operatorname{deg}\left(\left.\pi\right|_{C_{j}}\right)=1$ which we call horizontal and those with $\operatorname{deg}\left(\left.\pi\right|_{C_{j}}\right) \geq 2$ which we call slanted. We reorder again to obtain that $C_{1}, \ldots, C_{h}$, resp., $\Gamma_{1}, \ldots, \Gamma_{h}$, are the horizontal components of $C$, resp., $\Gamma$, and $C_{h+1}, \ldots, C_{k}$, resp., $\Gamma_{h+1}, \ldots, \Gamma_{k}$, are the slanted ones. An irreducible component of $C$ resp., $\Gamma$, which is not horizontal is referred to as a non-horizontal component; $C_{\text {horiz }}$ denotes the union of all horizontal components of $C$. In the same way we define $C_{\text {non-horiz }}, C_{\text {vert }}, C_{\text {non-vert }}, C_{\text {slant }}, \Gamma_{\text {horiz }}, \Gamma_{\text {non-horiz }}, D_{\text {horiz }}$, etc. Thus we have

$$
\Gamma_{\text {horiz }}=\bigcup_{i=1}^{h} \Gamma_{i}, \quad \Gamma_{\text {slant }}=\bigcup_{i=h+1}^{k} \Gamma_{i}, \quad \Gamma_{\text {vert }}=\bigcup_{i=k+1}^{n} \Gamma_{i},
$$

and similarly

$$
C_{\text {horiz }}=\bigcup_{i=1}^{h} C_{i}, \quad C_{\text {slant }}=\bigcup_{i=h+1}^{k} C_{i}, \quad C_{\text {vert }}=\bigcup_{i=k+1}^{n} C_{i} .
$$

2.8. Furthermore let $f=\prod_{j=1}^{n} f_{j}^{a_{j}}=f_{\text {horiz }} \cdot f_{\text {non-horiz }}$ be factorizations such that

$$
f_{j}^{*}(0)=D_{j}^{\text {red }}(j=1, \ldots, n), \quad\left(f_{\text {horiz }}\right)^{-1}(0)=D_{\text {horiz }} \simeq \Gamma_{\text {horiz }} \times \mathbb{C}
$$

and

$$
\left(f_{\text {non-horiz }}\right)^{-1}(0)=D_{\text {non-horiz }} \simeq \Gamma_{\text {non-horiz }} \times \mathbb{C} .
$$

2.9. An irreducible component is called isolated if it is a connected component. 
Let us give a typical example which illustrates our definitions.

Example 2.10. Let $Y=\mathbb{C}^{3}$ with coordinates $x, y, z$, and set $f=x y, g=y+x z$. Then $X=\{x y u+y+x z=0\} \subset \mathbb{C}^{4}=Y \times \mathbb{C}, D=\{x y=0\} \subset \mathbb{C}^{3}, \sigma(x, y, z, u)=$ $(x, y, z)$ and $E=\{x y=y+x z=0\} \subseteq \mathbb{C}^{4}$. Therefore, $\Gamma=\{x y=0\} \subset \mathbb{C}^{2}, \Gamma_{\text {horiz }}=$ $\{y=0\}$ and $\Gamma_{\text {vert }}=\{x=0\}$ whereas $C=C_{\text {vert }} \cup C_{\text {horiz }}$ with $C_{\text {horiz }}=\{y=z=0\}$ and $\Gamma_{\text {vert }}=\{x=y=0\}$.

The main result of this subsection is the following theorem.

Theorem 2.11. Let $X$ and $Y$ be smooth acyclic affine 3-folds satisfying the conditions (ii) and (iii) of 2.4. Then (in the notation of 2.6 2.9) the following hold:

( $\alpha) \pi \mid\left(C_{\text {horiz }} \backslash C_{\text {non-horiz }}\right): C_{\text {horiz }} \backslash C_{\text {non-horiz }} \rightarrow \Gamma_{\text {horiz }} \backslash \Gamma_{\text {non-horiz }}$ is an isomorphism.

( $\beta$ ) The slanted components $C_{h+1}, \ldots, C_{k}$ and $\Gamma_{h+1}, \ldots, \Gamma_{k}$ are isolated and homeomorphic to $\mathbb{C}$.

$(\gamma) f_{\text {non-horiz }}=p \circ f_{h+1}$ with $p \in \mathbb{C}[z]$, and every non-horizontal component of $\Gamma$ is homeomorphic to the affine line $\mathbb{C}$. In other words,

$$
f_{\text {non-horiz }}=c \cdot \prod_{i=h+1}^{n}\left(f_{h+1}-\lambda_{i}\right)^{a_{i}} \quad\left(c \in \mathbb{C}^{*}, \lambda_{h+1}=0\right)
$$

with $\pi\left(f_{h+1}^{-1}\left(\lambda_{i}\right)\right)=\Gamma_{i}$ homeomorphic to $\mathbb{C}(i=h+1, \ldots, n)$.

$(\delta)$ Up to a further reordering, the multiplicity matrix has the following form:

$$
M_{\sigma}=\left(\begin{array}{c|c|c}
I_{h} & 0 & B_{0} \\
--- & --- & --- \\
0 & I_{k-h} & 0 \\
--- & --- & --- \\
0 & 0 & I_{n-k}
\end{array}\right)
$$

Consequently (by 1.5), $\sigma_{*}: \pi_{1}(X) \rightarrow \pi_{1}(Y)$ is an isomorphism. Moreover, $X$ is contractible (and hence diffeomorphic to $\mathbb{R}^{6}$ ) if and only if $Y$ is also.

The rest of this subsection is devoted to the proof of Theorem[2.11, It is convenient to introduce the following terminology and notation.

2.12. Let $F$ be a curve. We say that a point $P \in F$ is multibranch (resp., unibranch) if it is a center of $\mu_{P}=\mu_{P}(F)>1$ (resp., $\mu_{P}=1$ ) local analytic branches of $F$. We denote by $F^{\text {norm }}$ the normalization of $F$ and by $\bar{F}$ its smooth complete model. The points of $\bar{F} \backslash F^{\text {norm }}$ are called the punctures of $F$. A morphism of curves $\rho: F \rightarrow G$ can be lifted to the normalizations, resp., the completions; we denote the lift by $\rho^{\text {norm }}: F^{\text {norm }} \rightarrow \Gamma^{\text {norm }}$, resp., $\bar{\rho}: \bar{F} \rightarrow \bar{G}$.

2.13. Consider further an irreducible smooth curve $F$ of genus $g$ with $n$ punctures, and let the 1-cycles $a_{1}, \ldots, a_{g}, b_{1}, \ldots, b_{g}$ on $F$ provide a symplectic basis of the group $H_{1}(\bar{F})=H_{1}(\bar{F} ; \mathbb{Z})$. Then there is an injection $H_{1}(\bar{F}) \hookrightarrow H_{1}(F)$ onto the subgroup generated by the classes $\left[a_{1}\right], \ldots,\left[a_{g}\right],\left[b_{1}\right], \ldots,\left[b_{g}\right]$; we may identify the group $H_{1}(\bar{F})$ with its image in $H_{1}(F)$. The group $H_{1}(F)$ being freely generated by the classes $\left[a_{1}\right], \ldots,\left[a_{g}\right],\left[b_{1}\right], \ldots,\left[b_{g}\right],\left[c_{1}\right], \ldots,\left[c_{n-1}\right]$, where $c_{1}, \ldots, c_{n}$ are simple 1 -cycles around the punctures of $F$, we have a (non-canonical) decomposition

$$
H_{1}(F) \simeq H_{1}(\bar{F}) \oplus G(F) \text { with } \quad G(F):=\left\langle\left[c_{1}\right], \ldots,\left[c_{n}\right]\right\rangle \simeq \mathbb{Z}^{n-1}
$$


$\left(\sum_{i=1}^{n}\left[c_{i}\right]=0\right.$ being the only relation between the generators $\left[c_{1}\right], \ldots,\left[c_{n}\right]$ of the group $G(F))$.

2.14. We denote by $S_{\Gamma}=S_{\Gamma}^{(0)} \cup S_{\Gamma}^{(1)}$ (where $S_{\Gamma}^{(0)} \cap S_{\Gamma}^{(1)}=\emptyset$ ) the finite subset of the curve $\Gamma$ such that a point $P$ belongs to $S_{\Gamma}$ if and only if it satisfies at least one of the following three conditions:

(i) $P=\pi\left(C_{i}\right)$ for a vertical component $C_{i}$ of $C\left(\Leftrightarrow P \in S_{\Gamma}^{(1)}\right)$;

(ii) $P=\pi(Q)$ for a multibranch point $Q$ of $C$;

(iii) $P$ is a multibranch point of $\Gamma$.

2.15. Set $S_{C}=\pi^{-1}\left(S_{\Gamma}\right) \subseteq C$ and $S_{C}^{(i)}=\pi^{-1}\left(S_{\Gamma}^{(i)}\right), i=0,1$. Thus the analytic set $S_{C}$ contains the union $S_{C}^{(1)}=C_{\text {vert }}$ of vertical components of $C$, whereas the residue set $S_{C}^{(0)}=S_{C} \backslash S_{C}^{(1)}$ is finite.

It is easily seen that if $Q \in S_{C}^{(0)}$, then over any local analytic branch $B_{i}$ of $\Gamma$ at the point $P:=\pi(Q)$ there is at least one local analytic branch $A_{j} \subseteq \pi^{-1}\left(B_{i}\right) \cap D_{g}^{\text {red }}$ of $C$ at $Q$. Indeed, the surfaces $\pi^{-1}\left(B_{i}\right) \simeq B_{i} \times \mathbb{C}$ and $D_{g}$ meet at $Q$, and so the polynomial $\left.g\right|_{\pi^{-1}\left(B_{i}\right)} \in \mathcal{O}\left[B_{i}\right][z]$ vanishes at $Q=:\left(P, z_{0}\right)$, but its specialization at $P$ is non-zero, as $Q \in S_{C}^{(0)}$ means that no vertical component of $C$ passes through $Q$. Consequently $\mu_{Q}(C) \geq \mu_{P}(\Gamma)$ and $P \notin \Gamma_{\text {vert }}$, hence $\pi^{-1}\left(\Gamma_{\text {vert }}\right) \subseteq S_{C}^{(1)}=C_{\text {vert }}$.

2.16. We let $\Gamma^{*}=\Gamma \backslash S_{\Gamma}$ (more generally, $\left.\Gamma_{\text {something }}^{*}=\Gamma_{\text {something }} \cap \Gamma^{*}\right)$ and $C^{*}=$ $C \backslash S_{C}=\pi^{-1}\left(\Gamma^{*}\right)=\pi^{-1}\left(\Gamma_{\text {non-vert }}^{*}\right) \subseteq C$.

2.17. For a complex hermitian manifold $M$ and a closed analytic subset $T$ of $M$, by a $l i n k l k_{P}(T)$ of $T$ at a point $P \in T$ we mean the intersection $T \cap S_{\varepsilon}$ of $T$ with a small enough sphere $S_{\varepsilon}$ in $M$ centered at $P$. We also call link the corresponding homology class $\left[l k_{P}(T)\right] \in H_{*}(T \backslash\{P\})=H_{*}(T \backslash\{P\} ; \mathbb{Z})$, and we still denote it simply by $l k_{P}(T)$.

2.18. We denote by $H_{\Gamma}$, resp., $H_{C}$, the subgroup of the group $H_{1}\left(\Gamma^{*}\right)=H_{1}\left(\Gamma^{*} ; \mathbb{Z}\right)$,

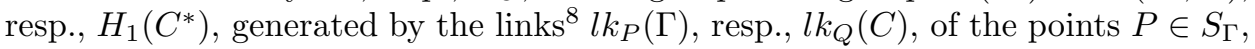
resp., $Q \in S_{C}^{(0)}$. Notice that $l k_{P}(\Gamma)=\sum_{i=1}^{\mu} l k_{P}\left(B_{i}\right)$, where $B_{1}, \ldots, B_{\mu}$ are the local branches of $\Gamma$ at $P$ and $\mu:=\mu_{P}(\Gamma)$. Clearly, $H_{\Gamma} \subseteq G\left(\Gamma^{*}\right)$, resp., $H_{C} \subseteq G\left(C^{*}\right)$, and so we obtain (non-canonical) isomorphisms

$$
H_{1}\left(C^{*}\right) / H_{C} \simeq H_{1}\left(\overline{C^{*}}\right) \oplus\left[G\left(C^{*}\right) / H_{C}\right],
$$

resp.,

$$
H_{1}\left(\Gamma^{*}\right) / H_{\Gamma} \simeq H_{1}(\bar{\Gamma}) \oplus\left[G\left(\Gamma^{*}\right) / H_{\Gamma}\right] .
$$

The next proposition is our main technical tool in the proof of Theorem 2.11.

Proposition 2.19. Under the assumptions as in 2.4, consider the restriction $\pi=$ $\pi_{\mid C^{*}}: C^{*} \rightarrow \Gamma^{*}$. Then $\pi_{*}\left(H_{C}\right) \subseteq H_{\Gamma}$, and $\pi$ induces the following isomorphisms:

$$
\begin{aligned}
& \widehat{\pi}_{*}: H_{1}\left(C^{*}\right) / H_{C} \cong H_{1}\left(\Gamma^{*}\right) / H_{\Gamma}, \\
& \bar{\pi}_{*}: H_{1}\left(\overline{C^{*}}\right) \stackrel{\cong}{\cong} H_{1}(\bar{\Gamma}), \\
& \tilde{\pi}_{*}: G\left(C^{*}\right) / H_{C} \stackrel{\cong}{\longrightarrow} G\left(\Gamma^{*}\right) / H_{\Gamma} .
\end{aligned}
$$

\footnotetext{
${ }^{8}$ Here we are in the special case when $M=X$ resp., $Y$ and $T=C$ resp. $\Gamma$.
} 
The proof is based on Lemmas 2.21 and 2.22 below. Let us introduce the following notation.

2.20. Denote

$$
S_{E}=\sigma^{-1}\left(S_{C}\right)=S_{C} \times \mathbb{C} \subseteq E, \quad \text { resp. }, \quad S_{E}^{(i)}=\sigma^{-1}\left(S_{C}^{(i)}\right)(i=0,1),
$$

and

$$
S_{D}=\pi^{-1}\left(S_{\Gamma}\right) \subseteq D, \quad \text { so that } \quad S_{D} \simeq S_{\Gamma} \times \mathbb{C} \subseteq \Gamma \times \mathbb{C} \simeq D .
$$

Furthermore, set

$E^{*}=\sigma^{-1}\left(C^{*}\right)=E \backslash S_{E}=C^{*} \times \mathbb{C}, \quad$ resp.,$\quad D^{*}=\pi^{-1}\left(\Gamma^{*}\right)=D \backslash S_{D} \simeq \Gamma^{*} \times \mathbb{C}$,

and

$$
X^{*}=X \backslash S_{E}, \quad \text { resp., } \quad Y^{*}=Y \backslash S_{D} .
$$

Thus

$$
X \backslash E=X^{*} \backslash E^{*}, \quad \text { resp., } \quad Y \backslash D=Y^{*} \backslash D^{*},
$$

and so we have an isomorphism

$$
\left.\sigma\right|_{X^{*} \backslash E^{*}}: X^{*} \backslash E^{*} \rightarrow Y^{*} \backslash D^{*} .
$$

Lemma 2.21. There are monomorphisms

$$
\rho_{X}: H_{3}\left(X^{*}\right) \rightarrow H_{1}\left(C^{*}\right), \quad \text { resp., } \quad \rho_{Y}: H_{3}\left(Y^{*}\right) \rightarrow H_{1}\left(\Gamma^{*}\right),
$$

such that $\pi_{*} \rho_{X}=\rho_{Y} \sigma_{*}$ and

$$
\widehat{\pi}_{*}: H_{1}\left(C^{*}\right) / \rho_{X}\left(H_{3}\left(X^{*}\right)\right) \rightarrow H_{1}\left(\Gamma^{*}\right) / \rho_{Y}\left(H_{3}\left(Y^{*}\right)\right)
$$

is an isomorphism.

Proof. The map of pairs $\left.\sigma\right|_{X^{*}}:\left(X^{*}, X^{*} \backslash E^{*}\right) \rightarrow\left(Y^{*}, Y^{*} \backslash D^{*}\right)$ induces the following commutative diagram (where the horizontal lines are exact homology sequences of pairs):

$\left(*_{1}\right)$

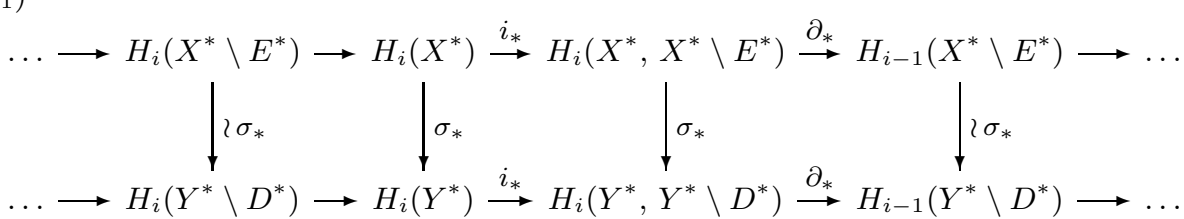

Claim. (a) $H_{3}\left(X^{*} \backslash E^{*}\right)=H_{3}\left(Y^{*} \backslash D^{*}\right)=0$ and (b) $H_{2}\left(X^{*}\right)=H_{2}\left(Y^{*}\right)=0$.

Proof of the claim. (a) By the isomorphism

$$
\sigma_{*}: H_{3}\left(X^{*} \backslash E^{*}\right)=H_{3}(X \backslash E) \stackrel{\cong}{\longrightarrow} H_{3}(Y \backslash D)=H_{3}\left(Y^{*} \backslash D^{*}\right)
$$

it is enough to show the vanishing of one of these groups, say, of $H_{3}(X \backslash E)$. As before, let $\dot{F}$ denote the one-point compactification of a topological space $F$. As $X$ is acyclic (hence by 1.12 the Alexander duality can be applied to $\dot{X}$ ) and moreover $E$ is closed in $X$, the Alexander duality gives an isomorphism

$$
H_{3}(X \backslash E) \cong H^{2}(\dot{E},\{\infty\}) .
$$

Consider the homeomorphisms $E \approx C \times \mathbb{R}^{2}, \dot{E} \approx\left(\dot{C} \times \dot{\mathbb{R}}^{2}\right) /\left(\dot{C} \vee \dot{\mathbb{R}}^{2}\right)$ and replace $\dot{\mathbb{R}}^{2}$ by $S^{2}$. Since $\left(\dot{C} \times S^{2}, \dot{C} \vee S^{2}\right)$ has the homotopy type of a pair of cell complexes, by [6, 4.4] we get

$$
H^{2}(\dot{E},\{\infty\}) \cong \widetilde{H}^{2}(\dot{E}) \cong H^{2}\left(\dot{C} \times S^{2}, \dot{C} \vee S^{2}\right)
$$


The Künneth formula for cohomology [26, (11.2)] yields a monomorphism

$$
\mu: \sum_{p+q=2} H^{p}(\dot{C},\{\infty\}) \otimes H^{q}\left(S^{2},\{\infty\}\right)=: H \rightarrow H^{2}\left(\dot{C} \times S^{2}, \dot{C} \vee S^{2}\right)
$$

with the cokernel

$$
\operatorname{coker} \mu=\sum_{p+q=3} \operatorname{Tor}\left(H^{p}(\dot{C},\{\infty\}), H^{q}\left(S^{2},\{\infty\}\right)\right) .
$$

As

$$
H^{0}(\dot{C},\{\infty\})=H^{0}\left(S^{2},\{\infty\}\right)=H^{1}\left(S^{2},\{\infty\}\right)=0
$$

we have $H=0$. The group $H^{*}\left(S^{2},\{\infty\}\right)$ being torsion free, we also have coker $\mu=$ 0 , and so $H^{2}\left(\dot{C} \times S^{2}, \dot{C} \vee S^{2}\right)=0$ as well. Thus in view of (15), $H_{3}(X \backslash E)=0$. This proves (a).

(b) By the Alexander duality we obtain

$$
H_{2}\left(X^{*}\right)=H_{2}\left(X \backslash S_{E}\right) \cong H^{3}\left(\dot{S}_{E},\{\infty\}\right)=H^{3}\left(\dot{S}_{E}\right) .
$$

The topological space $\dot{S}_{E}$ is homeomorphic to a bouquet of 4 -spheres $S^{4}$ and 2spheres $S^{2}$. The 4 -spheres are provided by the one-point compactification of the product $S_{C}^{(1)} \times \mathbb{C}=C_{\text {vert }} \times \mathbb{C}$ (recall (see 2.6) that the components of the curve $C_{\text {vert }}$ are disjoint and each one is isomorphic to $\mathbb{C}$ ), whereas the 2 -spheres $S^{2}$ are provided by the one-point compactification of the product $S_{C}^{(0)} \times \mathbb{C}$.

Hence $H^{3}\left(\dot{S}_{E}\right) \cong H_{2}\left(X^{*}\right)=0$. Similarly, we have $H_{2}\left(Y^{*}\right)=0$. This proves the claim.

In virtue of the above claim, $\left(*_{1}\right)$ (with $i=3$ ) leads to the commutative diagram

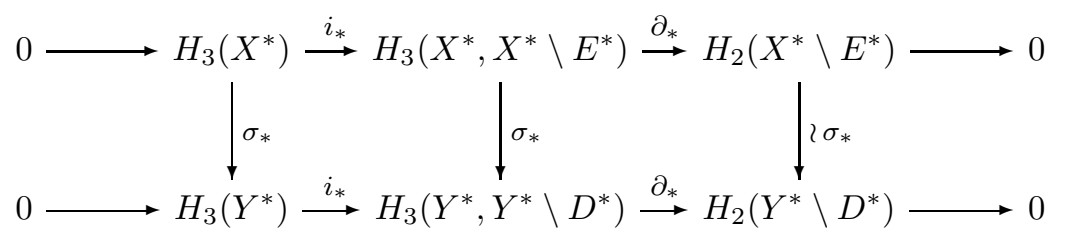

Next we apply the Thom isomorphism [6, 7.15] to the pairs of manifolds $\left(X^{*}, E^{*}\right)$, resp., $\left(Y^{*}, D^{*}\right)$ (cf. the proof of [1.9). Indeed, the curve $C^{*}$, resp., $\Gamma^{*}$, being locally unibranch (hence homeomorphic to its normalization), $E^{*}=C^{*} \times \mathbb{C}$, resp., $D^{*}=\Gamma^{*} \times \mathbb{C}$, is a topological manifold. Notice that (in virtue of 1.2 and 2.6) $\sigma^{*}$ maps the transverse classes [6, Ch. VIII] of $E^{*}$ into those of $D^{*}$. By functoriality of the cap-product [6, VII.12.6], for every $i \geq 2$ the following diagram is commutative:

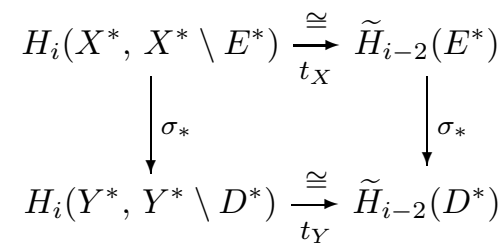


By making use of $\left(*_{3}\right)$ together with the following commutative diagram (see 2.5):

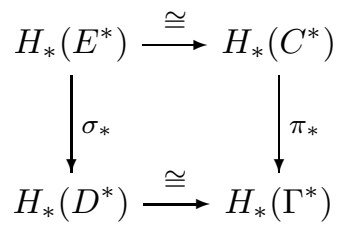

we may replace in $\left(*_{2}\right)$ the group $H_{3}\left(X^{*}, X^{*} \backslash E^{*}\right)$, resp., $H_{3}\left(Y^{*}, Y^{*} \backslash D^{*}\right)$, by the group $H_{1}\left(C^{*}\right)$, resp., $H_{1}\left(\Gamma^{*}\right)$, to obtain a commutative diagram

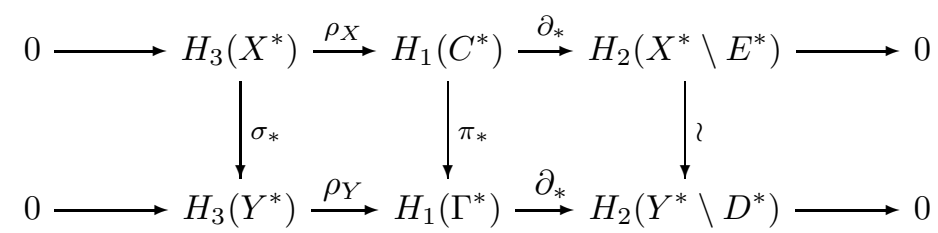

where $\rho_{X}:=\sigma_{*} \circ t_{X} \circ i_{*}$, resp., $\rho_{Y}:=\pi_{*} \circ t_{Y} \circ i_{*}$. The diagram $\left(*_{5}\right)$ yields the assertions of the lemma.

Lemma 2.22. $\rho_{X}\left(H_{3}\left(X^{*}\right)\right)=H_{C}$ and $\rho_{Y}\left(H_{3}\left(Y^{*}\right)\right)=H_{\Gamma}$.

Proof. We start by constructing an appropriate free base of the $\mathbb{Z}$-module $H_{3}\left(X^{*}\right)$ (resp., $H_{3}\left(Y^{*}\right)$ ). By the Alexander duality we have isomorphisms

$$
\widetilde{H}_{3}\left(X^{*}\right) \cong H^{2}\left(\dot{S}_{E}\right) \cong H^{2}\left(\dot{S}_{E}^{(0)}\right) \cong \mathbb{Z}^{b_{0}\left(S_{C}^{(0)}\right)},
$$

where $S_{E}^{(0)}:=S_{C}^{(0)} \times \mathbb{C}$ (similarly, $\left.\widetilde{H}_{3}\left(Y^{*}\right) \cong \mathbb{Z}^{b_{0}\left(S_{\Gamma}\right)}\right)$. Thus $H_{3}\left(X^{*}\right)=\widetilde{H}_{3}\left(X^{*}\right) \cong$ $H^{2}\left(\dot{S}_{E}^{(0)}\right)$ is a free $\mathbb{Z}$-module, and so the universal coefficient formula provides yet another form of the Alexander duality:

$$
H_{3}\left(X^{*}\right) \cong H^{2}\left(\dot{S}_{E}^{(0)}\right) \cong H_{2}\left(\dot{S}_{E}^{(0)}\right) \quad\left(\text { resp. }, \quad H_{3}\left(Y^{*}\right) \cong H_{2}\left(\dot{S}_{D}\right)\right)
$$

(see, e.g., [9, Ch. 2, §15.5]). Now a free base of $H_{3}\left(X^{*}\right)$ can be reconstructed as follows. On each component $T_{Q}:=\sigma^{-1}(Q)=\{Q\} \times \mathbb{C} \simeq \mathbb{C}$ of $S_{E}^{(0)}\left(\right.$ where $\left.Q \in S_{C}^{(0)}\right)$ fix a point $Q^{\prime} \in T_{Q}$, and fix a complex 2-ball $B_{Q^{\prime}}^{2}$ in $X$ transversal to $T_{Q}$ at $Q^{\prime}$. Then the 3 -cycle $\left[s_{Q^{\prime}}\right] \in H_{3}\left(X^{*}\right)$ that corresponds to the 2-cycle $\left[\dot{T}_{Q}\right] \in H_{2}\left(\dot{S}_{E}^{(0)}\right)$ by the Alexander duality, can be represented by a small 3 -sphere (say) $s_{Q^{\prime}}$ in $B_{Q^{\prime}}^{2}$ centered at $Q^{\prime}[9$, Ch. 2, $\S 17]$. These cycles $\left[s_{Q^{\prime}}\right] \in H_{3}\left(X^{*}\right)$ with $\pi\left(Q^{\prime}\right)=Q$, where $Q$ runs over $S_{C}^{(0)}$, form the desired base.

It is convenient to fix the choice of a point $Q^{\prime} \in T_{Q}$ as follows. Consider the curve $C^{\prime} \subseteq X$ given by the equations $u=f \circ \sigma=0$ (thus $C^{\prime}=E \cap D_{u}^{\text {red }}$ ). It is easily seen that the restriction $\sigma \mid C^{\prime}: C^{\prime} \rightarrow C$ is an isomorphism. Its inverse $C \rightarrow C^{\prime} \subseteq E$ is a section of the projection $\sigma \mid E: E=C \times \mathbb{C} \rightarrow C$. Letting $Q^{\prime}:=T_{Q} \cap C^{\prime}$ and placing the transversal ball $B_{Q^{\prime}}^{2}$ into the divisor $D_{u}^{\text {red }}=\{u=0\} \subseteq X$ (notice that by 2.3, $Q^{\prime}$ is a smooth point of $D_{u}^{\text {red }} \simeq D_{g}^{\text {red }}$ ), we let $\delta_{Q^{\prime}}:=s_{Q^{\prime}} \cap C^{\prime}$. Then $\sigma\left(\delta_{Q^{\prime}}\right)=: \delta_{Q}=l k_{Q}(C)$ is a link of the curve $C$ at the point $Q \in C$, and so $\delta_{Q} \in H_{C}$. We claim that $\rho_{X}\left(\left[s_{Q^{\prime}}\right]\right)=\left[\delta_{Q}\right] \in H_{1}\left(C^{*}\right)$, or in other words, that $i_{*}\left(\left[s_{Q^{\prime}}\right]\right)=t_{X}^{-1}\left(\left[\delta_{Q^{\prime}}\right]\right)$.

Indeed, consider the Zariski open dense subsets $X^{* *}:=X^{*} \backslash \operatorname{sing} E^{*} \subseteq X^{*}$, resp., $\operatorname{reg} E^{*}:=E^{*} \backslash \operatorname{sing} E^{*} \subseteq E^{*}$. Let $U$ be a small tubular neighborhood of the closed 
submanifold reg $E^{*} \subseteq X^{* *}$ with a retraction $\tau: U \rightarrow \operatorname{reg} E^{*}$. We have $\delta_{Q^{\prime}} \subseteq \operatorname{reg} E^{*}$, and the class $\left[\tau^{-1}\left(\delta_{Q^{\prime}}\right)\right] \in H_{3}\left(X^{* *}, X^{* *} \backslash \operatorname{reg} E^{*}\right)=H_{3}\left(U, U \backslash \operatorname{reg} E^{*}\right)$ corresponds to the class $\left[\delta_{Q^{\prime}}\right]$ under the Thom isomorphism $H_{3}\left(X^{* *}, X^{* *} \backslash \operatorname{reg} E^{*}\right) \stackrel{\cong}{\longrightarrow} H_{1}\left(\operatorname{reg} E^{*}\right)$ [9, Ch. $4, \S 30]$. The Thom isomorphism being functorial under open embeddings [6, VIII.11.5], we have $t_{X}^{-1}\left(\left[\delta_{Q^{\prime}}\right]\right)=\left[\tau^{-1}\left(\delta_{Q^{\prime}}\right)\right] \in H_{3}\left(X^{*}, X^{*} \backslash E^{*}\right)$.

The divisors $D_{u}^{\text {red }}$ and $E^{*}$ in $X^{*}$ are transversal, and so the 3 -sphere $s_{Q^{\prime}}$ is transversal to the divisor $E^{*}$ along the 1-cycle $\delta_{Q^{\prime}} \subseteq E^{*}$. Hence $s_{Q^{\prime}} \cap U$ and $\tau^{-1}\left(\delta_{Q^{\prime}}\right)$ represent the same relative homology class in

$$
H_{3}\left(U, U \backslash \operatorname{reg} E^{*}\right)=H_{3}\left(X^{* *}, X^{* *} \backslash \operatorname{reg} E^{*}\right) .
$$

This proves the equality $\rho_{X}\left(\left[s_{Q^{\prime}}\right]\right)=\left[\delta_{Q}\right]$.

Therefore, we have shown that $\rho_{X}\left(H_{3}\left(X^{*}\right)\right) \subseteq H_{C}$, and every element $\left[\delta_{Q}\right]$ of a free base of the $\mathbb{Z}$-module $H_{C}$ is the image of an element $\left[s_{Q^{\prime}}\right] \in H_{3}\left(X^{*}\right)$ with $\sigma\left(Q^{\prime}\right)=Q$. This proves the first equality of the lemma; the proof of the second one is similar.

Proof of Proposition 2.19. By 2.21 and 2.22 we have $\pi_{*}\left(H_{C}\right) \subseteq H_{\Gamma}$ and $\hat{\pi}_{*}$ in (11) is, indeed, an isomorphism. Furthermore, as $H_{1}(\bar{\Gamma})=H_{1}(\Gamma) / G\left(\Gamma^{*}\right)$ and $H_{1}\left(\overline{C^{*}}\right)=$ $H_{1}(C) / G\left(C^{*}\right)$ (see 2.13) , the homomorphism $\bar{\pi}_{*}: H_{1}\left(\overline{C^{*}}\right) \rightarrow H_{1}(\bar{\Gamma})$ factors through $\widehat{\pi}_{*}$, hence $\bar{\pi}_{*}$ is a surjection. To show that it is also injective, consider a non-zero element $[\alpha] \in H_{1}\left(\overline{C^{*}}\right)$ generated by a 1-cycle $\alpha$ in $C_{i}^{*}$ (where $i \leq k$ ) such that the 1cycle $\beta:=\pi(\alpha)$ in $\Gamma_{i}^{*}$ gives a zero element of $H_{1}\left(\bar{\Gamma}_{i}\right)$. Hence the class $[\beta] \in H_{1}\left(\Gamma_{i}^{*}\right)$ is contained in the subgroup $G\left(\Gamma_{i}^{*}\right)$. It is easily seen that $\pi_{*}\left(G\left(C_{i}^{*}\right)\right)$ contains a subgroup of finite index in $G\left(\Gamma_{i}^{*}\right)$. Thus for some $m>0$ we have $m[\beta]=\pi_{*}([\gamma])$ with $[\gamma] \in G\left(C_{i}^{*}\right)$. As $m[\alpha]-[\gamma]$ is still a non-zero element of $H_{1}\left(\overline{C^{*}}\right)$ whose image in $H_{1}\left(\Gamma_{i}^{*}\right)$ is zero, we have $\widehat{\pi}_{*}(m[\alpha]-[\gamma])=0$, which is wrong since $\widehat{\pi}_{*}$ is an isomorphism. This proves that $\bar{\pi}_{*}$ in (12) is an isomorphism.

It follows that the decompositions (91) and (10) in 2.18 can be chosen in such a way that $\pi_{*}$ respects them. Then by (11) and (12), $\tilde{\pi}_{*}$ in (13) is also an isomorphism. The proof is completed.

2.23. The proof of the next lemma is based on the following simple observation. Let $G$ is a free abelian group of finite rank. For an element $a \in G$, the following conditions are equivalent:

(i) $a$ is primitive (that is, if $a=k b$ with $b \in G, k \in \mathbb{Z}$, then $k= \pm 1$ );

(ii) given a free base $a_{1}, \ldots, a_{n}$ of a $\mathbb{Z}$-module $G$, the coordinates in the presentation $a=\sum_{i=1}^{n} k_{i} a_{i}$ are relatively prime.

Lemma 2.24. Let $F$ be a curve with the irreducible components $F_{1}, \ldots, F_{L}$, which are all non-compact, and with the multiple points $P_{1}, \ldots, P_{M}$. Denote by $p_{1}, \ldots, p_{N}$ the punctures of the curve $F^{*}:=F \backslash\left\{P_{1}, \ldots, P_{M}\right\}$, and let $B_{i}$ be a local branch of $\bar{F}$ centered at $p_{i}$. Consider the group $G:=G\left(F^{*}\right) / H_{F}$, where

$$
G\left(F^{*}\right):=\left\langle l k_{p_{i}}\left(B_{i}\right) \mid i=1, \ldots, N\right\rangle \subseteq H_{1}\left(F^{*}\right)
$$

and

$$
H_{F}:=\left\langle l k_{P_{j}}(F) \mid j=1, \ldots, M\right\rangle \subseteq G\left(F^{*}\right) .
$$

Then $G \cong \mathbb{Z}^{N-M-L}$. Furthermore, for each $i=1, \ldots, N$ the class $\left[l k_{p_{i}}\left(B_{i}\right)\right] \in G$ is primitive unless it is zero. The latter holds if and only if $p_{i}$ is the only puncture of the corresponding component $F_{l}$, and $F_{l}$ is an isolated component of $F$. 
Proof. Denoting $\nu: F^{\text {norm }} \rightarrow F$ a normalization, we let $\nu^{-1}\left(P_{m}\right)=\left\{p_{j}\right\}_{j \in J_{m}}$ with $J:=\coprod_{m=1}^{M} J_{m} \subseteq\{1, \ldots, N\} 9$ Thus $l k_{P_{m}}(F)=\sum_{j \in J_{m}} l k_{p_{j}}\left(B_{j}\right)$. We also let $\left\{p_{i}\right\}_{i \in I_{l}}$ be the set of punctures of a component $F_{l}^{*}:=F_{l} \cap F^{*}$ of the curve $F^{*}$. For every $m=1, \ldots, M$ (resp., $l=1, \ldots, L$ ) we pick an index $j_{m} \in J_{m}$ (resp., $i_{l} \in I_{l} \backslash J$ ) (notice that the index set $I_{l} \backslash J$ of the punctures of $F_{l}$ is non-empty, as $F_{l}$ is assumed to be non-compact). Then we have

$$
\begin{gathered}
G\left(F^{*}\right)=\left\langle l k_{p_{i}}\left(B_{i}\right) \mid i \notin\left\{i_{1}, \ldots, i_{L}\right\}\right\rangle \\
=\left\langle l k_{p_{i}}\left(B_{i}\right), l k_{P_{m}}(F) \mid m=1,, \ldots, M, \quad i \notin\left\{i_{1}, \ldots, i_{L}, j_{1}, \ldots, j_{M}\right\}\right\rangle .
\end{gathered}
$$

Thus $G\left(F^{*}\right) \cong \mathbb{Z}^{N-L} \cong H_{F} \oplus \mathbb{Z}^{N-L-M}$ with $H_{F} \cong \mathbb{Z}^{M}$, and so

$$
G=G\left(F^{*}\right) / H_{F}=\left\langle\left[l k_{p_{i}}\left(B_{i}\right)\right] \mid i \notin\left\{i_{1}, \ldots, i_{L}, j_{1}, \ldots, j_{M}\right\}\right\rangle \cong \mathbb{Z}^{N-L-M} .
$$

The elements $\left[l k_{p_{i}}\left(B_{i}\right)\right] \in G$ of the free base (16) satisfy condition (ii) of 2.23 , hence are primitive. The indices $j_{m} \in J_{m}$ being arbitrary, as card $J_{m} \geq 2$, the classes $\left[l k_{p_{i}}\left(B_{i}\right)\right] \in G$ with $i \in J$ are all primitive as well. By the same reason, a class $\left[l k_{p_{i}}\left(B_{i}\right)\right] \in G$ with $i \in I_{l} \backslash J$ is primitive unless $\{i\}=I_{l} \backslash J$ (i.e., unless $p_{i}$ is the only puncture of $F_{l}$ ). If such a component $F_{l}$ meets another one $F_{l^{\prime}}$ at a multibranch point $P_{m}$, then choosing $j_{m}$ from $J_{m} \cap I_{l^{\prime}}$ and decomposing $\left[l k_{p_{i}}\left(B_{i}\right)\right] \in G$ in the base (16) we obtain that at least one of the coordinates equals -1 , hence 2.23)(ii) is fulfilled, and so $\left[l k_{p_{i}}\left(B_{i}\right)\right]$ is primitive too. Finally, $\left[l k_{p_{i}}\left(B_{i}\right)\right] \in G$ is not primitive if and only if $p_{i}$ is the only puncture of $F_{l}$ and $F_{l}$ is isolated (in that case $l k_{p_{i}}\left(B_{i}\right)+\sum_{P_{m} \in F_{l}} l k_{P_{m}}(F)=0$ in $G\left(F^{*}\right)$, hence $\left[l k_{p_{i}}\left(B_{i}\right)\right]=0$ in $\left.G\right)$.

In the proof of Theorem 2.11 below (based on 2.19 and 2.24) the role of $F$ in 2.24 is played respectively by the curves $\Gamma$ and $C_{\text {non-vert }} \backslash C_{\text {vert }}$. As in the proof of 2.24, it will be important to bear in mind the freedom of choice when selecting the indices $\left\{i_{1}, \ldots, i_{L}, j_{1}, \ldots, j_{M}\right\}$ as in (16). From 2.24 we obtain such a corollary.

Corollary 2.25. In the notation as in 2.19, let $Q \in \bar{C}_{i}(i \in\{1, \ldots, k\})$ be a puncture of $C_{i}^{*}$ such that $P:=\bar{\pi}(Q) \in \bar{\Gamma}_{i}$ is not a puncture at infinity of the affine curve $\Gamma_{i}$. Then the following hold:

(a) mult $_{Q} \bar{\pi}=1$ (that is, $\bar{\pi}$ is non-ramified at $Q$ );

(b) $P$ is a puncture of $\Gamma_{i}^{*}$;

(c) the components $C_{i}$ and $\Gamma_{i}$ are horizontal (that is, $1 \leq i \leq h$ ).

Proof. The curve $C_{i}^{*}$ possesses yet another puncture over a place at infinity of $\Gamma_{i}$, hence by (2.24), $\left[l k_{Q}\left(C_{i}^{*}\right)\right]$ is a primitive element of the group $G\left(C^{*}\right) / H_{C}$. As $\tilde{\pi}_{*}$ in (13) is an isomorphism, $\tilde{\pi}_{*}\left(\left[l k_{Q}\left(C_{i}^{*}\right)\right]\right)=\kappa\left[l k_{P}\left(\Gamma_{i}^{*}\right)\right] \in G\left(\Gamma^{*}\right) / H_{\Gamma} \backslash\{0\}$ is primitive as well, hence $\kappa=\operatorname{mult}_{Q} \bar{\pi}=1$, which yields (a) and (b).

Suppose that there are two different local branches $A$ and $A^{\prime}$ of $\bar{C}$ over a local branch $B$ of $\bar{\Gamma}$ at $P$, and let $Q, Q^{\prime} \in \bar{C}$ be their centers. The same argument as above shows that $\tilde{\pi}_{*}\left(\left[l k_{Q}(A)\right]\right)=\tilde{\pi}_{*}\left(\left[l k_{Q^{\prime}}\left(A^{\prime}\right)\right]\right)=\left[l k_{P}(B)\right]$, where both $\left[l k_{Q}(A)\right],\left[l k_{Q^{\prime}}\left(A^{\prime}\right)\right] \in G\left(C^{*}\right) / H_{C}$ are primitive. Hence

$$
\left[l k_{Q}(A)\right]=\left[l k_{Q^{\prime}}\left(A^{\prime}\right)\right] \neq 0 .
$$

It is not difficult to verify that (in contradiction with (17)) there exists a base (16) which includes both $\left[l k_{Q}(A)\right]$ and $\left[l k_{Q^{\prime}}\left(A^{\prime}\right)\right]$, unless $A$ and $A^{\prime}$ are local branches of the curve $C$ at a multibranch point $Q \in S_{C}^{(0)}$ with $\mu_{Q}(C)=2$. But in the latter

\footnotetext{
${ }^{9}$ Here $\amalg$ stands for the disjoint union.
} 
case $\left[l k_{Q}(A)\right]=-\left[l k_{Q^{\prime}}\left(A^{\prime}\right)\right]$, which again contradicts (17). Therefore, there is only one local branch of $\bar{C}$ over $B$, which yields (c).

Now we are ready to prove Theorem 2.11

Proof of Theorem 2.11. It is convenient to proceed first with the proof of $(\beta)$. From 2.25 we get

$$
\Gamma_{\text {slant }} \cap S_{\Gamma}=\emptyset=C_{\text {slant }} \cap S_{C}
$$

(indeed, $\Gamma_{\text {slant }}^{*}$, resp., $C_{\text {slant }}^{*}$, cannot have punctures other than the places at infinity). Thus the slanted components $C_{i}$ and $\Gamma_{i}(h+1 \leq i \leq k)$ of $C$, resp., $\Gamma$, are isolated and do not contain multibranch points. In particular, $C_{i}=C_{i}^{*}$, resp., $\Gamma_{i}=\Gamma_{i}^{*}$, and these curves are homeomorphic to their normalizations. Furthermore, the group $G\left(C_{i}^{*}\right)$ (resp., $G\left(\Gamma_{i}^{*}\right)$ ) is a direct summand of $G\left(C^{*}\right) / H_{C}$ (resp., $\left.G\left(\Gamma^{*}\right) / H_{\Gamma}\right)$. Hence by 2.19 ,

$$
\left.\tilde{\pi}_{*}\right|_{G\left(C_{i}^{*}\right)}: G\left(C_{i}^{*}\right) \stackrel{\cong}{\longrightarrow} G\left(\Gamma_{i}^{*}\right)
$$

is an isomorphism, as well as

$$
\left.\bar{\pi}_{*}\right|_{H_{1}\left(\bar{C}_{i}\right)}: H_{1}\left(\bar{C}_{i}\right) \stackrel{\cong}{\longrightarrow} H_{1}\left(\bar{\Gamma}_{i}\right) .
$$

Since $\left.\operatorname{deg} \bar{\pi}\right|_{\bar{C}_{i}}>1$, (19) shows that $H_{1}\left(\bar{C}_{i}\right)=0=H_{1}\left(\bar{\Gamma}_{i}\right)$, i.e., both $\bar{C}_{i}$ and $\bar{\Gamma}_{i}$ are rational curves. Therefore by (86) in 2.13

$$
H_{1}\left(C_{i}^{\text {norm }}\right) \cong H_{1}\left(C_{i}\right)=H_{1}\left(C_{i}^{*}\right)=G\left(C_{i}^{*}\right)
$$

and

$$
H_{1}\left(\Gamma_{i}^{\text {norm }}\right) \cong H_{1}\left(\Gamma_{i}\right)=H_{1}\left(\Gamma_{i}^{*}\right)=G\left(\Gamma_{i}^{*}\right) .
$$

Now (18) yields that

$$
\pi_{*}^{\text {norm }}: H_{1}\left(C_{i}^{\text {norm }}\right) \rightarrow H_{1}\left(\Gamma_{i}^{\text {norm }}\right)
$$

is an isomorphism. By a theorem of Hurwitz (see, e.g., [22, I.2.1]), a morphism $\rho: F \rightarrow G$ of smooth irreducible affine curves is an isomorphism once the induced homomorphism $\rho_{*}: H_{1}(F) \rightarrow H_{1}(G)$ is an isomorphism, unless $H_{1}(F)=H_{1}(G)=$ 0, i.e., $F \simeq G \simeq \mathbb{C}$. Thus $C_{i}^{\text {norm }} \simeq \Gamma_{i}^{\text {norm }} \simeq \mathbb{C}$, and so the curves $C_{i}$ and $\Gamma_{i}$ are homeomorphic to $\mathbb{C}$, which proves $(\beta)$.

$(\alpha)$ By $2.15 \pi^{-1}\left(\Gamma_{\text {vert }}\right) \subseteq C_{\text {vert }}$, hence $\pi\left(C_{\text {horiz }} \backslash C_{\text {vert }}\right) \subseteq \Gamma_{\text {horiz }} \backslash \Gamma_{\text {vert }}$. To show that the latter inclusion is actually an equality, suppose on the contrary that for a point $P \in \Gamma_{\text {horiz }} \backslash \Gamma_{\text {vert }}, \pi^{-1}(P)=\emptyset$. Then all $\mu:=\mu_{P}(\Gamma)$ branches $A_{1}, \ldots, A_{\mu}$ of $\bar{C}$ over the branches $B_{1}, \ldots, B_{\mu}$ of $\Gamma$ at $P$ have centers at infinity. By 2.25(b) the curve $\Gamma^{*}$ has a puncture over $P$. Thus $P \in S_{\Gamma}^{(0)}$ is a multibranch point of $\Gamma_{\text {horiz }} \backslash \Gamma_{\text {vert }}$, and so the primitive classes (see 2.24$)\left[l k_{P}\left(B_{j}\right)\right] \in G\left(\Gamma^{*}\right) / H_{\Gamma}(j=1, \ldots, \mu)$ are subjected to the (only) relation $\sum_{j=1}^{\mu}\left[l k_{P}\left(B_{j}\right)\right]=0$. Therefore, the primitive classes $\left[l k_{Q_{j}}\left(A_{j}\right)\right] \in G\left(C^{*}\right) / H_{C}(j=1, \ldots, \mu)$ are also related by

$$
\sum_{j=1}^{\mu}\left[l k_{Q_{j}}\left(A_{j}\right)\right]=0,
$$

where $Q_{j} \in \bar{C}$ is the center of the branch $A_{j}$. Constructing a free base (16) of the free $\mathbb{Z}$-module $G\left(C^{*}\right) / H_{C}$, we may suppose that for any $j=1, \ldots, \mu, j \notin$ $\left\{i_{1}, \ldots, i_{L}\right\}$ (and definitely, $j \notin\left\{j_{1}, \ldots, j_{M}\right\}$ as $Q_{j}$ is a puncture at infinity of $C$ ). Thus the classes $\left[l k_{Q_{j}}\left(A_{j}\right)\right](j=1, \ldots, \mu)$ make a part of a free base (16), and so cannot satisfy (20), a contradiction. 
Denote $F:=C_{\text {horiz }} \backslash C_{\text {non-horiz }}=C_{\text {horiz }} \backslash C_{\text {vert }}$ and $G:=\Gamma_{\text {horiz }} \backslash \Gamma_{\text {non-horiz }}=$ $\Gamma_{\text {horiz }} \backslash \Gamma_{\text {vert }}$. We have shown that the morphism $\pi_{\mid F}: F \rightarrow G$ of degree 1 is bijective. It follows that it is an isomorphism. Indeed, as $D_{g}$ and $D_{f}$ meet transversally along $F$ (see 2.5), the curve $F$ is the zero divisor of the restriction $\left.g\right|_{G \times \mathbb{C}_{z}}$, which is a polynomial of degree one in $z$, say, $a z+b \in \mathbb{C}[G][z]$. Note that $a$ and $b$ have no common zero on $G$ (otherwise the zeros of $\left.g\right|_{G \times \mathbb{C}}=a z+b$ would contain a vertical component). As $\left.\pi\right|_{F}$ is surjective, $a$ is nowhere zero, hence $z=-b / a \in \mathbb{C}[G]$, and so $\left.\pi\right|_{F}$ is an isomorphism. This proves $(\alpha)$.

$(\gamma)$ The group $G\left(\Gamma^{*}\right) / H_{\Gamma}=\tilde{\pi}_{*}\left(G\left(C^{*}\right) / H_{C}\right)$ being generated by the classes

$$
\tilde{\pi}\left(\left[l k_{Q_{j}}\left(C_{i}^{*}\right)\right]\right) \in G\left(\Gamma_{\text {non-vert }}^{*}\right) /\left[H_{\Gamma} \cap G\left(\Gamma_{\text {non-vert }}^{*}\right)\right]
$$

(where $Q_{j}$ runs over the set of punctures of the curve $C^{*}$ ), we have

$$
G\left(\Gamma_{\text {non-vert }}^{*}\right) /\left[H_{\Gamma} \cap G\left(\Gamma_{\text {non-vert }}^{*}\right)\right]=G\left(\Gamma^{*}\right) / H_{\Gamma} .
$$

Since, by $(\beta), G\left(\Gamma_{\text {slant }}^{*}\right)=0$, we obtain

$$
G\left(\Gamma^{*}\right) / H_{\Gamma}=G\left(\Gamma_{\text {non-slant }}^{*}\right) / H_{\Gamma}=G\left(\Gamma_{\text {horiz }}^{*}\right) /\left[H_{\Gamma} \cap G\left(\Gamma_{\text {horiz }}^{*}\right)\right] .
$$

In particular, for each puncture $P$ of $\Gamma_{\text {vert }}^{*}$, we have

$$
\left[l k_{P}(\bar{\Gamma})\right] \in G\left(\Gamma_{\text {horiz }}^{*}\right) /\left[H_{\Gamma} \cap G\left(\Gamma_{\text {horiz }}^{*}\right)\right] .
$$

It follows that on any vertical component of $\Gamma$ there is only one puncture at infinity. Indeed, if there were a component $\Gamma_{i}$ of $\Gamma_{\text {vert }}$ with at least two punctures at infinity, say, $P_{1}$ and $P_{2}$, then any free base as in (16) of the $\mathbb{Z}$-module $G\left(\Gamma^{*}\right) / H_{\Gamma}$ would contain at least one of the corresponding classes, say, $\left[l k_{P_{1}}(\bar{\Gamma})\right]$, which contradicts (21).

A similar argument shows that the curve $\Gamma_{\text {vert }}$ has no selfintersection. In particular, the components of $\Gamma_{\text {vert }}$ are disjoint, and for each $i=k+1, \ldots, n, \Gamma_{i}$ is homeomorphic to $\Gamma_{i}^{\text {norm }}$.

Furthermore, by 2.19

$$
\bar{\pi}_{*}: H_{1}\left(\bar{C}^{*}\right) \rightarrow H_{1}(\bar{\Gamma})=H_{1}\left(\bar{\Gamma}_{\text {non-vert }}\right) \oplus H_{1}\left(\bar{\Gamma}_{\text {vert }}\right)
$$

is an isomorphism, and $\bar{\pi}_{*}\left(H_{1}\left(\bar{C}^{*}\right)\right) \subseteq H_{1}\left(\bar{\Gamma}_{\text {non-vert }}\right)$. It follows that $H_{1}\left(\bar{\Gamma}_{\text {vert }}\right)=0$, hence the components of $\Gamma_{\text {vert }}$ are rational. Finally, $\Gamma_{i}(i=k+1, \ldots, n)$ is a rational curve with one place at infinity and without selfintersections, therefore is homeomorphic to $\mathbb{C}$.

As for any $i=h+2, \ldots, n, D_{h+1} \cap D_{i}=\emptyset$ and $D_{i} \simeq \Gamma_{i} \times \mathbb{C}$ is simply connected, with the notation as in 2.6 we have that the restriction $\left.f_{h+1}\right|_{D_{i}}$ does not vanish, and so is constant: $\left.f_{h+1}\right|_{D_{i}}=: \lambda_{i} \in \mathbb{C}$. Thus we obtain a decomposition as in $(\gamma)$, which completes the proof of $(\gamma)$.

$(\delta)$ As the matrix $B^{\prime}$ in 2.6 is unimodular and by $(\gamma)$ the vertical components are disjoint, arguing as in 2.6 we can easily see that the morphism $\pi: C \rightarrow \Gamma$ maps any component of $C_{\text {vert }}$ into a component of $\Gamma_{\text {vert }}$, and maps different components of $C_{\text {vert }}$ into different components of $\Gamma_{\text {vert }}$. Moreover, the columns of $B^{\prime}$ are vectors of the standard basis in $\mathbb{R}^{n-k}$. Hence up to a reordering we may assume that $B^{\prime}$ is the unit matrix. Since the slanted components $\Gamma_{h+1}, \ldots, \Gamma_{k}$ are isolated, the last $k-h$ lines of the matrix $B$ are zero, which completes the proof of $(\delta)$.

Remark 2.26. Notice that for any vertical component $C_{i}$ of $C(i=k+1, \ldots, n)$, $P_{i}:=\pi\left(C_{i}\right)$ is a smooth point of the curve $\Gamma_{i}$. This follows from[1.2 as $m_{i i}=1$. 
2.3. Preserving acyclicity: a criterion. We have the following partial converse to 2.11

Theorem 2.27. Let $X$ and $Y$ be irreducible smooth affine 3-folds which satisfy condition (ii) of 2.4 as well as the following (which replaces 2.4(iii)):

(iii') $Y=Z \times \mathbb{C}$ is a cylinder over a smooth acyclic affine surface $Z$, and $D=$ $\Gamma \times \mathbb{C}$ with $\Gamma \subseteq Z$.

Suppose also that the conditions $(\alpha)-(\delta)$ of Theorem 2.11 are fulfilled 10 Then the induced homomorphisms

$$
\sigma_{*}: H_{*}(X) \rightarrow H_{*}(Y) \quad \text { and } \quad \sigma_{*}: \pi_{1}(X) \rightarrow \pi_{1}(Y)
$$

are isomorphisms. Henceforth, the 3 -fold $X$ is acyclic; it is contractible if and only if $Y$ is also.

Proof. The theorem immediately follows from 1.4 1.10 and 2.32 below. Indeed, the assumption 2.11 $(\delta)$ allows to apply 1.4 in order to get that $\sigma_{*}: \pi_{1}(X) \rightarrow \pi_{1}(Y)$ is an isomorphism. In turn, by 2.32 the assumptions of 1.10 are fulfilled, and so by 1.10, $\sigma_{*}: H_{*}(X) \rightarrow H_{*}(Y)$ is an isomorphism as well.

From 2.11 and 2.27 we obtain the following criterion for preserving the acyclicity.

Corollary 2.28. Let $X$ and $Y$ be smooth affine 3-folds satisfying conditions 2.4(ii) and [2.27(iii'). If $Y$ is acyclic, resp., contractible, then $X$ is so if and only if conditions 2.11 $(\alpha)-(\delta)$ are fulfilled.

Remark 2.29. Assuming in 2.27 that the conditions $2.11(\alpha)-(\delta)$ are fulfilled we require implicitly that the things are as in 2.6!2.8, without supposing acyclicity as in [2.4(i). In fact, 2.6-2.8 refer only to the fact that the multiplicity matrix $M_{\sigma}$ is unimodular, which is anyhow foreseen by $2.11(\delta)$.

Actually the proofs of the lemmas below rely only on conditions (ii), (iii) and the following:

(iv) There is a regular function $\varphi \in A=\mathbb{C}[Y]$ such that for every $i=h+1, \ldots, n$ the restriction $\left.\varphi\right|_{D_{i}}$ is constant, as well as the restriction of $\varphi$ to any fiber of the morphism $\pi: D \rightarrow \Gamma$, and for any point $P \in S_{\Gamma} \backslash \Gamma_{\text {vert }}$, both $\Phi_{P}:=$ $\varphi^{*}\left(\varphi\left(\pi^{-1}(P)\right)\right) \subseteq Y$ and $\Psi_{P}:=\sigma^{-1}\left(\Phi_{P}\right) \subseteq X$ are smooth, reduced surfaces with $\sigma^{*}\left(\Phi_{P}\right)=\Psi_{P}$.

Under these assumptions $\sigma_{*}$ is an isomorphism in homology (even if we do not suppose as in 2.27 that $Y$ is acyclic).

The next lemma shows that the conditions (ii) and (iii') imply (iv).

Lemma 2.30. Under the assumptions of 2.27 there exists a (possibly empty) smooth, reduced curve $\Gamma^{\prime}$ on $Z$ such that

(a) $\Gamma^{\prime} \cap \Gamma_{\text {non-horiz }}=\emptyset$ and $\Gamma^{\prime} \supseteq S_{\Gamma}^{(0)} \backslash \Gamma_{\text {non-horiz }}$,

(b) the surfaces $\Phi:=\Gamma^{\prime} \times \mathbb{C} \subseteq Y$ and $\Psi:=\sigma^{-1}(\Phi) \subseteq X$ are smooth, and

(c) $\left.\sigma\right|_{\Psi}: H_{*}(\Psi) \rightarrow H_{*}(\Phi)$ is an isomorphism.

Proof. If $h<n$ (i.e., $\Gamma_{\text {non-horiz }} \neq \emptyset$ ), then we take for $\Gamma^{\prime}$ the union of the fibers of the regular function $\left.f_{h+1}\right|_{Z}$ through the points of $S_{\Gamma}^{(0)} \backslash \Gamma_{\text {non-horiz }}$. Indeed, since the Euler characteristics $e\left(\Gamma_{h+1}\right)=e(Z)=1$ and $\Gamma_{h+1}=\left(f_{h+1} \mid Z\right)^{*}(0)$, by [42.

\footnotetext{
${ }^{10}$ See 2.29 below.
} 
6.2] the fibers of $\left.f_{h+1}\right|_{z}$ are smooth, reduced and irreducible except for, possibly, $\Gamma_{h+1}$. Thus $\Gamma^{\prime}$ is smooth and reduced. As $f_{h+1}$ is constant on any component $\Gamma_{i}$ of $\Gamma_{\text {non-horiz }}$ we have $\Gamma^{\prime} \cap \Gamma_{\text {non-horiz }}=\emptyset$, hence (a) is fulfilled.

In the case where $h=n$ (i.e., $\Gamma_{\text {non-horiz }}=\emptyset$ ) the existence of a smooth, reduced curve $\Gamma^{\prime}$ satisfying (a) easily follows by Bertini's Theorem.

Evidently, $\Phi=\Gamma^{\prime} \times \mathbb{C}$ is a smooth surface. Since $\left.\sigma\right|_{X \backslash E}: X \backslash E \rightarrow Y \backslash D$ is an isomorphism, the surface $\Psi:=\sigma^{-1}(\Phi)$ is smooth if it is smooth at the points $R \in \Psi \cap E$. Let $Q=\left(P, z_{0}\right):=\sigma(R) \in C$ with $P \in \Gamma \cap \Gamma^{\prime} \subseteq \Gamma_{\text {horiz }} \backslash \Gamma_{\text {non-horiz. }}$. As $\Gamma^{\prime}$ is smooth we can find local coordinates $(x, y)$ on $Z$ centered at $P$ such that (locally) $\Gamma^{\prime}=\{x=0\}$. Thus $Q=\left(P, z_{0}\right)=\left(0,0, z_{0}\right), R=\left(Q, u_{0}\right)=\left(0,0, z_{0}, u_{0}\right)$, and (locally)

$$
\Psi=\{x=0\} \cap X=\{f(0, y) u-g(0, y, z)=0\} \subseteq \mathbb{C}_{y, z, u}^{3},
$$

where by condition $2.11(\alpha)$, (locally) $g(0, y, z)=a(y) z+b(y)$ with $a(0) \neq 0$ (see the proof of $2.11(\alpha))$. Therefore, $\partial g / \partial z(Q)=a(0) \neq 0$, hence the surface $\Psi$ is smooth at $R$. Thus (b) holds.

To show (c) we need the following claim.

Claim. $\left(\left.\sigma\right|_{\Psi}\right)^{*}\left(\left(D_{\text {horiz }} \cap \Phi\right)^{\text {red }}\right)=\left(E_{\text {horiz }} \cap \Psi\right)^{\text {red }}$.

Proof of the Claim. We will use the same local chart and the notation as above. We have $\left(D_{\text {horiz }} \cap \Phi\right)^{\text {red }}=\{P\} \times \mathbb{C}_{z}=y^{*}(0)$ ( $y$ being regarded as a function on $\Phi)$. Then locally, $(y \circ \sigma):(y, z, u) \longmapsto y$, hence the subspace $\left.\operatorname{ker} d(y \circ \sigma)\right|_{R}=\{y=$ $0\}$ does not contain the tangent space $T_{R} X$ (indeed as $a(0) \neq 0$ the differential $\left.d(f u-g)\right|_{R}=\ldots-a(0) d z$ of the defining polynomial of $\Psi$ in $\mathbb{C}_{y, z, u}^{3}$ at the point $R$ is not proportional to the differential $d y$ of $y=y \circ \sigma$ at $R$ ). It follows that $\left.d(y \circ \sigma)\right|_{\Psi}$ does not vanish at the points $R \in \Psi \cap E$, and so locally

$$
\left(E_{\text {horiz }} \cap \Psi\right)^{\text {red }}=(y \circ \sigma)^{*}(0)=\left(\left.\sigma\right|_{\Psi}\right)^{*}\left(\left(D_{\text {horiz }} \cap \Phi\right)^{\text {red }}\right),
$$

as desired.

Notice that $\Phi \cap D_{\text {horiz }}=\left(\Gamma^{\prime} \cap \Gamma_{\text {horiz }}\right) \times \mathbb{C} \subseteq \Phi$ is a disjoint union of affine lines, whereas in virtue of $2.11(\alpha), \Phi \cap C_{\text {horiz }}$ is a finite set of points, one on each of those lines. Furthermore, $\Psi \cap E_{\text {horiz }}=\left(\Phi \cap C_{\text {horiz }}\right) \times \mathbb{C}$.

The proof of (c) is based on 1.9. In the notation as in 1.9 we let $\hat{X}:=\Psi, \hat{Y}:=$ $\Phi, \hat{E}:=\Psi \cap E_{\text {horiz }}$ and $\hat{D}:=\Phi \cap D_{\text {horiz }}$. Then by (b) above, $\hat{X}, \hat{Y}, \hat{E}$ and $\hat{D}$ are smooth varieties, $\sigma(\hat{E}) \subseteq \hat{D}, \sigma(\hat{X} \backslash \hat{E})=\hat{Y} \backslash \hat{D}$, and (by the above claim) $\sigma^{*}(\hat{D})=$ $\hat{E}$, that is, the condition (i) of 1.9 is fulfilled. As $\left.\sigma\right|_{\hat{X} \backslash \hat{E}}: \hat{X} \backslash \hat{E} \rightarrow \hat{Y} \backslash \hat{D}$ is an isomorphism, taking into account the observations following the claim it is easily seen that [1.9](ii) holds as well. Thus by[1.9] $\sigma_{*}: H_{*}(\hat{X}) \rightarrow H_{*}(\hat{Y})$ is an isomorphism, which yields (c).

2.31. The proof of the next lemma relies on 1.10 where we let $\hat{E}=E^{\prime} \cup E^{\prime \prime}$ and $\hat{D}=D^{\prime} \cup D^{\prime \prime}$ with $E^{\prime}:=E_{\text {horiz }}, D^{\prime}:=D_{\text {horiz }}$,

$$
E^{\prime \prime}:=E_{\text {non-horiz }} \coprod \Psi \text { and } \quad D^{\prime \prime}:=D_{\text {non-horiz }} \coprod \Phi,
$$

$\Phi$ and $\Psi$ being the same as in 2.30 (b).

Lemma 2.32. Under the assumptions and the notation as in 2.27 and 2.31, conditions $\left.\left(\mathrm{i}^{\prime}\right)\right)$ and $\left(\mathrm{ii}^{\prime}\right)$ of 1.10 are fulfilled. 
Proof. By $2.11(\gamma)$, for every $i=h+1, \ldots, n$ both curves $C_{i}$ and $\Gamma_{i}$ are homeomorphic to $\mathbb{C}$, hence both surfaces $E_{i}=C_{i} \times \mathbb{C}$ and $D_{i} \simeq \Gamma_{i} \times \mathbb{C}$ are homeomorphic to $\mathbb{C}^{2}$. Therefore $E_{\text {non-horiz }}$ and $D_{\text {non-horiz }}$ are topological manifolds, and $\left(\left.\sigma\right|_{E_{\text {non-horiz }}}\right)_{*}: H_{*}\left(E_{\text {non-horiz }}\right) \rightarrow H_{*}\left(D_{\text {non-horiz }}\right)$ is an isomorphism. As the surfaces $\Phi$ and $\Psi$ are smooth we have that $E^{\prime \prime}$ and $D^{\prime \prime}$ are topological manifolds as well, and in view of $2.30(\mathrm{c}),\left(\left.\sigma\right|_{E^{\prime \prime}}\right)_{*}: H_{*}\left(E^{\prime \prime}\right) \rightarrow H_{*}\left(D^{\prime \prime}\right)$ is an isomorphism.

As by our construction

$$
S_{\Gamma} \subseteq \Gamma^{\prime} \cup \Gamma_{\text {non-horiz }} \quad \text { and } \quad S_{C} \subseteq \pi^{-1}\left(\Gamma^{\prime}\right) \cup C_{\text {non-horiz }},
$$

the curves $\Gamma_{\text {horiz }} \backslash\left(\Gamma^{\prime} \cup \Gamma_{\text {non-horiz }}\right)$ and $C_{\text {horiz }} \backslash\left(\pi^{-1}\left(\Gamma^{\prime}\right) \cup C_{\text {non-horiz }}\right)$ have no multibranch points. Therefore they are topological manifolds, as well as the surfaces

$$
D^{\prime} \backslash D^{\prime \prime}=\left[\Gamma_{\text {horiz }} \backslash\left(\Gamma^{\prime} \cup \Gamma_{\text {non-horiz }}\right)\right] \times \mathbb{C}
$$

and

$$
E^{\prime} \backslash E^{\prime \prime}=\left[C_{\text {horiz }} \backslash\left(\pi^{-1}\left(\Gamma^{\prime}\right) \cup C_{\text {non-horiz }}\right)\right] \times \mathbb{C} .
$$

$\operatorname{By} 2.11(\alpha)$ the projection

$$
\pi: C_{\text {horiz }} \backslash\left(\pi^{-1}\left(\Gamma^{\prime}\right) \cup C_{\text {non-horiz }}\right) \rightarrow \Gamma_{\text {horiz }} \backslash\left(\Gamma^{\prime} \cup \Gamma_{\text {non-horiz }}\right)
$$

is an isomorphism, hence $\left.\sigma\right|_{E^{\prime} \backslash E^{\prime \prime}}: E^{\prime} \backslash E^{\prime \prime} \rightarrow D^{\prime} \backslash D^{\prime \prime}$ is so as well. Now the conditions $\left(\mathrm{i}^{\prime}\right)$ and $\left(\mathrm{ii}^{\prime}\right)$ of 1.10 follow.

\section{Simple AfFine modifications of $\mathbb{C}^{3}$ Diffeomorphic to $\mathbb{R}^{6}$}

The main result of this section is 3.6 which together with 2.28 provides a criterion for when a simple affine modification $X \subseteq \mathbb{C}^{4}$ of $Y=\mathbb{C}^{3}$ is isomorphic to $\mathbb{C}^{3}$.

3.1. Exotic simple modifications of $\mathbb{C}^{3}$. We keep the terminology and the notation of section 2 and we adopt the following

Convention 3.1. Hereafter

(i) $Y=\mathbb{C}^{3}$ with the coordinates $x, y, z$, and

(ii) $X$ is a smooth affine 3 -fold in $\mathbb{C}^{4}$ diffeomorphic to $\mathbb{R}^{6}$, with equation of the form

$$
p=f(x, y) u+g(x, y, z)=0,
$$

where $f \in \mathbb{C}[x, y] \backslash \mathbb{C}, g \in \mathbb{C}[x, y, z]$ (thus by 2.28, the conditions $2.11(\alpha)$ $(\delta)$ hold).

3.2. Note that the blowup morphism $\sigma: X \ni(x, y, z, u) \longmapsto(x, y, z) \in Y$ represents $X$ as a simple affine modification of $Y=\mathbb{C}^{3}$ along the cylindrical divisor $D=D_{f}^{\text {red }}=\Gamma \times \mathbb{C}\left(\right.$ where $\left.\Gamma:=f^{-1}(0) \subset \mathbb{C}^{2}\right)$ with center $C=\{f=g=0\} \subset \mathbb{C}^{3}$ and with the morphism $\pi: C \rightarrow \Gamma$ as in 2.5 given by $\pi:(x, y, z) \longmapsto(x, y)$. Hence the assumptions (i)-(iii) of 2.4 are fulfilled.

3.3. As in 2.8 we factorize $f \in \mathbb{C}[x, y]$ into irreducible factors: $f=\prod_{i=1}^{n} f_{i}^{a_{i}}$, and we write $g$ as a polynomial in $z$ :

$$
g(x, y, z)=\sum_{j=0}^{d} b_{j}(x, y) z^{j} \quad \text { with } \quad d:=\operatorname{deg}_{z} g .
$$

We let $d_{i}:=\operatorname{deg}\left(\left.\pi\right|_{C_{i}}\right)$. Recall (2.6, 2.7) that an irreducible component $C_{i}$ (resp., $\left.\Gamma_{i}=f_{i}^{-1}(0)\right)$ of the curve $C$ (resp., $\Gamma$ ) is vertical (resp., horizontal, resp., slanted) 
if and only if $d_{i}=0$ (resp., $d_{i}=1$, resp., $d_{i} \geq 2$ ). The following lemma is a simple observation, and so we omit the proof.

Lemma 3.4. For every $i=1, \ldots, n$ we have $b_{j} \in\left(f_{i}\right) \forall j=d_{i}+1, \ldots, d$ and $b_{d_{i}} \notin\left(f_{i}\right)$. Hence $p$ as in (22) admits a unique presentation

$$
p=f u+g_{i}+f_{i} h_{i}
$$

with $g_{i}(x, y, z):=\sum_{j=0}^{d_{i}} b_{j}(x, y) z^{j} \in \mathbb{C}[x, y, z]$ and $h_{i} \in\left(z^{d_{i}+1}\right) \subseteq \mathbb{C}[x, y, z]$. Furthermore, if $f_{\text {horiz }} \neq$ const, then $p$ can be written as

$$
p=f_{\text {horiz }} f_{\text {non-horiz }} u+b_{0}+b_{1} z+f_{\text {horiz }}^{\text {red }} h_{0}
$$

with $b_{0}, b_{1} \in \mathbb{C}[x, y]$ and $h_{0} \in\left(z^{2}\right) \subseteq \mathbb{C}[x, y, z]$, where $\left.b_{1}\right|_{\Gamma_{\text {horiz }} \backslash \Gamma_{\text {non-horiz }}}$ has no zero.

3.5. Recall 43 that an exotic $\mathbb{C}^{3}$ is a smooth affine 3 -fold diffeomorphic to $\mathbb{R}^{6}$ but non-isomorphic to $\mathbb{C}^{3}$.

The principal result of this subsection is the following theorem.

Theorem 3.6. If under the assumptions as in 3.1, at least one of the curves $C_{\text {non-horiz }}$ and $\Gamma_{\text {non-horiz }}$ is singular, then $X$ is an exotic $\mathbb{C}^{3}$.

The proof is done in 3.9 and 3.15 below. For a converse result, see 3.21 in the next subsection.

Notice that 3.6 provides a regular way of constructing exotic $\mathbb{C}^{3}$ 's as hypersurfaces in $\mathbb{C}^{4}$. Let us give concrete examples.

3.7. Examples. For the Russell cubic 3-fold

$$
X=\left\{x^{2} u+x+y^{2}+z^{3}=0\right\} \subset \mathbb{C}^{4},
$$

the curve $\Gamma=\Gamma_{\text {slant }}=\{x=0\} \subset \mathbb{C}^{2}$ is smooth and isomorphic to $\mathbb{C}$, whereas $C=C_{\text {slant }}=\left\{x=y^{2}+z^{3}=0\right\} \subset \mathbb{C}^{3}$ (the center of modification) is homeomorphic to $\mathbb{C}$ but singular. It is well known $23,5,43$ that $X$ represents an exotic algebraic structure on $\mathbb{C}^{3}$. By 15 the Koras-Russell cubic 3 -fold

$$
X=\left\{\left(x^{2}+y^{3}\right) u+x+z^{2}=0\right\} \subset \mathbb{C}^{4}
$$

also is an exotic $\mathbb{C}^{3}$; here both $\Gamma=\Gamma_{\text {slant }}=\left\{x^{2}+y^{3}=0\right\} \subset \mathbb{C}^{2}$ and $C=C_{\text {slant }}=$ $\left\{x^{2}+y^{3}=0=x+z^{2}\right\} \subset \mathbb{C}^{3}$ are homeomorphic to $\mathbb{C}$ but singular.

Remark 3.8. Generalizing a theorem of Sathaye [34, in [19 Thm. 7.2] it is proven that actually, every smooth acyclic surface in $\mathbb{C}^{3}$ with equation $p=f(x, y) u+$ $g(x, y)=0$ (where $f, g \in \mathbb{C}^{[2]}$ ) is isomorphic to $\mathbb{C}^{2}$ and rectifiable. Examples 3.7 show that in general, this does not hold anymore in $\mathbb{C}^{4}$ without the additional assumption of smoothness of $C_{\text {non-horiz }}$ and $\Gamma_{\text {non-horiz }}$ (cf. 3.6 above).

The proof of 3.6 starts with the following proposition (cf. 3.20 below).

Proposition 3.9. Let $X$ and $Y$ be as in 3.1. If the curve $\Gamma_{\text {non-horiz }}$ is singular, then the Derksen invariant $\operatorname{Dk}\left(A^{\prime}\right)$ of the algebra $A^{\prime}:=\mathbb{C}[X]$ is non-trivial, hence $X \not \mathbb{C}^{3}$.

The proof is done in $3.10+3.14$. 
Lemma 3.10. Under the assumptions as in [3.9, choosing appropriate new coordinates in the $(x, y)$-plane and rescaling the $z$-coordinate we may write the polynomial $p$ in the form

$$
p=\left(x^{k}-y^{l}\right)^{m} f_{\text {horiz }}(x, y) u+z^{e}+g_{0}(x, y, z)+\left(x^{k}-y^{l}\right) h_{0}(x, y, z),
$$

where $k, l, e \geq 2,(k, l)=1, f_{\text {horiz }} \in \mathbb{C}[x, y]$ and $f_{\text {horiz }}(0,0)=1, g_{0}, h_{0} \in$ $\mathbb{C}[x, y, z], \operatorname{deg}_{z} g_{0}<e$ and $z^{e} \mid h_{0}$.

Proof. In the notation as in 2.612.9 we have $h<n$. In virtue of condition 2.11 $(\gamma)$ we may suppose that the component $\Gamma_{h+1}$ of $\Gamma_{\text {non-horiz }}$ is a singular plane curve homeomorphic to $\mathbb{C}$. Hence by the Lin-Zaidenberg Theorem 22], choosing new coordinates in the $(x, y)$-plane we may assume that $f_{h+1}=x^{k}-y^{l}$ with $k, l \geq 2$ and $(k, l)=1$. As the other fibers $x^{k}-y^{l}=c(c \in \mathbb{C} \backslash\{0\})$ of the polynomial $f_{h+1}$ are not homeomorphic to $\mathbb{C}$, in view of $2.11(\gamma)$ we have $h+1=n$, and so $f_{\text {non-horiz }}=f_{n}^{m}=\left(x^{k}-y^{l}\right)^{m}$ with $m:=a_{n}$. By 3.4 the polynomial (22) can now be written as follows:

$$
p=\left(x^{k}-y^{l}\right)^{m} f_{\text {horiz }}(x, y) u+b_{e}(x, y) z^{e}+g_{0}(x, y, z)+\left(x^{k}-y^{l}\right) h_{0}(x, y, z)
$$

with $b_{e} \in \mathbb{C}[x, y], g_{0}, h_{0} \in \mathbb{C}[x, y, z], \operatorname{deg}_{z} g_{0}<e$ and $z^{e+1} \mid h_{0}$. In virtue of $2.11(\delta)$ and 2.5 the divisors $D_{n}=\Gamma_{n} \times \mathbb{C}$ and $D_{g}^{\text {red }}$ meet transversally at general points of $C_{n}=D_{n} \cap D_{g}^{\text {red }}$. If $\Gamma_{n}$ were vertical (i.e., $g(x, y, z) \equiv b_{0}(x, y) \bmod \left(x^{k}-y^{l}\right)$; see 3.3, 3.4), then the equation $b_{0}\left(t^{l}, t^{k}\right)=0$ would have a unique solution $t_{0}$ such that (by 2.26) $\pi\left(C_{n}\right)=\left(t_{0}^{l}, t_{0}^{k}\right)$ is a smooth point of $\Gamma_{n} \subseteq \mathbb{C}_{x, y}^{2}$, i.e., $t_{0} \neq 0$. Thus $b_{0}\left(t^{l}, t^{k}\right)=c\left(t-t_{0}\right)^{r}$ which is wrong as the derivative of the left-hand side vanishes at $t=0$. Therefore, $\Gamma_{n}=\Gamma_{\text {slant }}$, hence $e=\left.\operatorname{deg} \pi\right|_{C_{n}} \geq 2$ and $\left.\pi\right|_{C_{n}}$ : $C_{n} \rightarrow \Gamma_{n}$ is a proper morphism (as each of these curves has only one puncture). It follows that the restriction $\left.b_{e}\right|_{\Gamma_{n}}$ has no zero. As $\Gamma_{n}$ is simply connected we have $\left.b_{e}\right|_{\Gamma_{n}}=$ const $=: b_{e}^{0} \in \mathbb{C}^{*}$, and so $b_{e}(x, y)=b_{e}^{0}+\left(x^{k}-y^{l}\right) c_{e}(x, y)$ for a certain polynomial $c_{e} \in \mathbb{C}^{[2]}$. Finally, rescaling the $z$-coordinate if necessary, we obtain the desired presentation. As by $2.11(\delta), \Gamma_{n}=\Gamma_{\text {slant }}$ is an isolated component of $\Gamma$, the restriction $\left.f_{\text {horiz }}\right|_{\Gamma_{n}}$ does not vanish, hence is constant, and we may assume in addition that this constant is $f_{\text {horiz }}(0,0)=1$.

3.11. We choose the weight degree function $d$ on the algebra $A$ with

$$
d_{x}=-l N, d_{y}=-k N, d_{z}=\sqrt{2} \text { and } d_{u}=k \operatorname{lm} N+e \sqrt{2},
$$

so that the polynomials

$$
f_{n}=f_{h+1}=x^{k}-y^{l} \quad \text { and } \quad \hat{p}:=\left(x^{k}-y^{l}\right)^{m} u+z^{e}
$$

are $d$-quasihomogeneous. Letting $N \in \mathbb{N}$ be sufficiently large (thus $d\left(h_{0}\right)<k l N$, and hence $\left.d\left(\left(x^{k}-y^{l}\right) h_{0}\right)<0\right)$, we may assume that $\hat{p}$ as above is the $d$-principal part of the polynomial $p$ as in (24).

Lemma 3.12. Let $q \in \mathbb{C}[x, y, z, u] \backslash \mathbb{C}$ be an irreducible d-homogeneous polynomial with $\operatorname{deg}_{z} q<e$. Then $q$ coincides (up to a constant factor) with one of the following polynomials:

$$
x, y, z, u, \lambda x^{k}+\mu y^{l}, \quad \text { where } \quad \lambda, \mu \in \mathbb{C}^{*} .
$$


Proof. Letting $q=\sum_{i=0}^{e-1} a_{i}(x, y, u) z^{i}$ with $a_{i} \in \mathbb{C}[x, y, u](i=0, \ldots, e-1)$ we claim that there can be only one non-zero coefficient $a_{i}$. Assuming on the contrary that $a_{i}, a_{i+j} \neq 0$ for some $i, i+j \in\{0, \ldots, e-1\}$ with $1 \leq j \leq e-1$, we would have $d\left(a_{i} z^{i}\right)=d\left(a_{i+j} z^{i+j}\right)$. Taking into account the equality $d_{y}=\frac{k}{l} d_{x}$ we derive

$$
\begin{gathered}
d\left(a_{i} z^{i}\right)-d\left(a_{i+j} z^{i+j}\right)=j d_{z}=j \sqrt{2}=\alpha d_{u}+\beta d_{x}=\alpha e \sqrt{2}+\beta^{\prime} d_{x} \\
\Rightarrow(j-\alpha e) \sqrt{2}=\beta^{\prime} d_{x} \in \mathbb{Q}
\end{gathered}
$$

for certain $\alpha \in \mathbb{Z}_{\geq 0}$ and $\beta, \beta^{\prime} \in \mathbb{Q}$. But $\alpha e-j \in \mathbb{Q} \backslash\{0\}$, hence 25] leads to a contradiction.

Therefore, the irreducible polynomial $q$ must coincide (up to a constant factor) either with $z$ or with a polynomial $a_{0} \in \mathbb{C}[x, y, u]$. Let $q=a_{0}(x, y, u)=$ $\sum_{i=0}^{t} c_{i}(x, y) u^{i}$ with $c_{i} \in \mathbb{C}[x, y](i=0, \ldots, t)$. The weights $d_{x}$ (resp., $\left.d_{y}\right)$ and $d_{u}$ being independent over $\mathbb{Q}$, the same argument as above shows that at most one summand $c_{i}(x, y) u^{i}$ may be different from zero.

Thus once again, the irreducible polynomial $q$ coincides (up to a constant factor) either with $u$ or with a polynomial $c_{0} \in \mathbb{C}[x, y]$. In the latter case, being $d$-homogeneous the polynomial $q$ must coincide (up to a constant factor) with one of the polynomials $x, y, \lambda x^{k}+\mu y^{l}\left(\lambda, \mu \in \mathbb{C}^{*}\right)$, as stated.

3.13. Let an irreducible polynomial $p \in \mathbb{C}^{[4]}$ be as in (24). Set as before $X=$ $p^{-1}(0) \subset \mathbb{C}^{4}, \hat{X}=\hat{p}^{-1}(0) \subset \mathbb{C}^{4}$ and $\hat{A}^{\prime}=\mathbb{C}[\hat{X}]$, where $\hat{p}$ is the $d$-principal part of $p$. Denote by $\rho_{\hat{A}^{\prime}}$ the canonical surjection

$$
\rho_{\hat{A}^{\prime}}: \mathbb{C}^{[4]} \rightarrow \mathbb{C}^{[4]} /(\hat{p})=\hat{A}^{\prime}
$$

and by $\hat{x}=\rho_{\hat{A}^{\prime}}(x), \ldots, \hat{u}=\rho_{\hat{A}^{\prime}}(u) \in \hat{A}^{\prime}$ the traces on $\hat{X}$ of the coordinate functions.

The polynomial $\hat{p}$ being irreducible, by 1.22 (a) $\hat{A}^{\prime}$ is just the graded algebra associated with the filtered algebra $A^{\prime}$, a filtration being provided by the degree function $d$ on $A^{\prime}$.

Lemma 3.14. In the notation as in 3.13 we have

$$
\operatorname{ML}_{\mathrm{gr}}\left(\hat{A}^{\prime}\right)=\operatorname{Dk}_{\mathrm{gr}}\left(\hat{A}^{\prime}\right)=\mathbb{C}[\hat{x}, \hat{y}] \subset \hat{A}^{\prime} \leq 0 .
$$

Therefore (by 1.24)

$$
\operatorname{Dk}\left(A^{\prime}\right) \subset A_{0}^{\prime} \neq A^{\prime},
$$

so that $A^{\prime} \nsucceq \mathbb{C}^{[3]}$ and $X \nsucceq \mathbb{C}^{3}$, which proves 3.9.

Proof. The Jacobian derivation

$$
\partial_{0}:=\frac{\partial(\hat{p}, x, y, *)}{\partial(x, y, z, u)}
$$

on the algebra $\hat{A}^{\prime}$ being homogeneous and locally nilpotent with $\hat{A}^{\prime}{ }^{\partial_{0}}=\operatorname{ker} \partial_{0}=$ $\mathbb{C}[\hat{x}, \hat{y}]$, it is sufficient to show that $\hat{x}, \hat{y} \in \operatorname{ker} \hat{\partial}$ for any $\hat{\partial} \in \operatorname{LND}_{\mathrm{gr}}\left(\hat{A}^{\prime}\right)$. Indeed, by 1.14 both $\mathbb{C}[\hat{x}, \hat{y}]$ and ker $\hat{\partial}$ are algebraically closed subalgebras of $\hat{A}^{\prime}$ of transcendence degree 2 , thereby they coincide provided that $\mathbb{C}[\hat{x}, \hat{y}] \subset \operatorname{ker} \hat{\partial}$.

The derivation $\hat{\partial}$ being homogeneous, the subalgebra ker $\hat{\partial} \subset \hat{A}$ is generated by homogeneous elements (i.e., by the restrictions to $\hat{X}$ of $d$-homogeneous polynomials on $\mathbb{C}^{4}$ ). Let $a=\left.q\right|_{\hat{X}} \in \operatorname{ker} \hat{\partial}$ (with a $d$-homogeneous $q \in \mathbb{C}^{[4]}$ ) be non-constant. We may assume that $\operatorname{deg}_{z} q<e$ (otherwise we replace the polynomial $q$ by the 
rest of the Euclidean division of $q$ by the $z$-monic $d$-homogeneous polynomial $\hat{p}=$ $\left.z^{e}+\left(x^{k}-y^{l}\right)^{m} u\right)$.

The kernel $\hat{A}^{\hat{\partial}}=\operatorname{ker} \hat{\partial}$ being factorially closed (see 1.14(c)), the irreducible factors of $q$ restricted to $\hat{X}$ belong to this kernel as well. Therefore, ker $\hat{\partial}$ is generated by the traces of irreducible $d$-homogeneous polynomials $q$ with $\operatorname{deg}_{z} q<e$. By 1.14(d) we have $\hat{x}, \hat{y} \in \operatorname{ker} \hat{\partial}$ provided that $\lambda \hat{x}^{k}+\mu \hat{y}^{l} \in \operatorname{ker} \hat{\partial}$ for some $\lambda, \mu \in \mathbb{C}^{*}$. Due to 3.12 and to the above argument on algebraic closeness, ker $\hat{\partial}$ coincides with the subalgebra of $\hat{A}^{\prime}$ generated by one of the following pairs:

$$
(\hat{x}, \hat{y}), \quad(\hat{x}, \hat{u}), \quad(\hat{y}, \hat{u}), \quad(\hat{x}, \hat{z}), \quad(\hat{y}, \hat{z}), \quad(\hat{z}, \hat{u}) .
$$

If $\hat{z} \in \operatorname{ker} \hat{\partial}$, then by 1.14 and the equality

$$
\hat{z}^{e}+\left(\hat{x}^{k}+\hat{y}^{l}\right)^{m} \hat{u}=0
$$

$\hat{x}, \hat{y}, \hat{u} \in \operatorname{ker} \hat{\partial}$ also, hence $\hat{\partial}=0$, a contradiction. This eliminates the last three cases.

If ker $\hat{\partial}=\mathbb{C}[\hat{x}, \hat{u}], 11$ then (26) yields the equality

$$
l m \operatorname{deg}_{\hat{\partial}} \hat{y}=e \operatorname{deg}_{\hat{\partial}} \hat{z} .
$$

On the other hand, as $\operatorname{ker} \hat{\partial}=\mathbb{C}[\hat{x}, \hat{u}]$, by $1.19 \hat{\partial}$ is equivalent to the Jacobian derivation

$$
\hat{\partial}_{1}:=\frac{\partial(\hat{p}, x, u, *)}{\partial(x, y, z, u)} \text {. }
$$

It is easily seen that

$$
\hat{\partial}_{1}(\hat{y})=-\left.(\hat{p})_{z}^{\prime}\right|_{\hat{X}}=-\hat{z}^{e-1},
$$

and so as $\operatorname{deg}_{\partial}=\operatorname{deg}_{\partial_{1}}$ (see 1.18) we get

$$
\operatorname{deg}_{\hat{\partial}} \hat{y}=(e-1) \operatorname{deg}_{\hat{\partial}} \hat{z}+1 .
$$

From (27) and (28) we obtain

$$
\left[\left(1-\frac{1}{l m}\right) e-1\right] \operatorname{deg}_{\hat{\partial}} \hat{z}=-1 .
$$

But this is impossible because $l, e \geq 2, m \geq 1$, and so $\left(1-\frac{1}{l m}\right) e \geq 1$. This completes the proof of the lemma, as well as those of 3.9 .

Next we consider the remaining possibility in 3.6 .

Proposition 3.15. Let $X$ and $Y$ be as in 3.1. Suppose that $\Gamma_{\text {non-horiz }}$ is a smooth curve, whereas there exists a singular component, say, $C_{h+1}$, of the curve $C_{\text {slant }}$. Then once again, the Derksen invariant $\operatorname{Dk}\left(A^{\prime}\right)$ is non-trivial, hence $X \neq \mathbb{C}^{3}$.

The proof is done in 3.163 .19 below.

Lemma 3.16. Under the assumptions as in 3.15, after applying an appropriate tame automorphism of $\mathbb{C}_{x, y, z}^{3}$ the polynomial $p$ can be presented in the form

$$
p=x^{m} h_{0}(x, y, z) u+y^{k}+z^{l}+x h_{1}(x, y, z)
$$

with $k, l, m \geq 2,(k, l)=1, h_{0}, h_{1} \in \mathbb{C}[x, y, z]$ and $h_{0}(0, y, z)=$ const $=1$.

\footnotetext{
${ }^{11}$ The case where $\operatorname{ker} \hat{\partial}=\mathbb{C}[\hat{y}, \hat{u}]$ can be eliminated by a similar argument.
} 
Proof. The curve $\Gamma_{h+1} \subset \mathbb{C}_{x, y}^{2}$ (being smooth and homeomorphic to $\mathbb{C}$ ) is isomorphic to $\mathbb{C}$. Thus by the Abhyankar-Moh-Suzuki Theorem, choosing appropriate new coordinates in the $(x, y)$-plane we may suppose that $f_{h+1}=x$. We factorize $f=f_{h+1}^{m} \tilde{f}=x^{m} \tilde{f}$ with $m:=a_{h+1}$. As $C_{h+1}$ is singular, by 2.3 the gradient $\operatorname{grad} f$ has to vanish at some point of $\Gamma_{h+1}=\{x=0\}$. The component $\Gamma_{h+1}$ of $\Gamma$ being isolated $(\operatorname{see} 2.11 \beta)$ ) this implies that $m \geq 2$. Furthermore the polynomial $\tilde{f}(0, y)$ does not vanish, hence is a non-zero constant; by rescaling the $x$-coordinate we may assume this constant being 1 .

As the curve $C_{h+1}=\{x=0=g(0, y, z)\} \subseteq \mathbb{C}_{y, z}^{2}$ is homeomorphic to $\mathbb{C}$ and singular, by the Lin-Zaidenberg Theorem (after performing an appropriate automorphism of the plane $\mathbb{C}_{y, z}^{2}$ ) we may suppose that $C_{h+1}$ is given by $x=y^{k}+$ $z^{l}=0$ with $k, l \geq 2$ and $(k, l)=1$. By [2.5] and condition [2.11 $(\delta)$ the divisors $D_{h+1}=\{x=0\} \subset \mathbb{C}_{x, y, z}^{3}$ and $D_{g}^{\text {red }}$ meet transversally at general points of the curve $C_{h+1}$, hence $g(0, y, z)=y^{k}+z^{l}$ (up to a constant factor which can be put equal to 1$)$, i.e., $g=y^{k}+z^{l}+x h_{1}(x, y, z)$. Taking for $h_{0}(x, y, z)$ the polynomial obtained from $\tilde{f}$ as a result of the latter coordinate change, we obtain the desired presentation (29) with $h_{0}(0, y, z)=\tilde{f}(0, y)=1$.

3.17. We consider the weight degree function $d$ on the algebra $A^{\prime}:=\mathbb{C}[X]$ with

$$
d_{x}=-1, d_{y}=d_{z}=0 \quad \text { and } \quad d_{u}=m .
$$

As the $d$-principal part $\hat{p}=x^{m} u+y^{k}+z^{l}$ of the polynomial $p$ as in (29) is irreducible; by $1.22 \hat{A}^{\prime}:=\mathbb{C}[\hat{X}]$ is just the graded algebra associated with the filtration on $A^{\prime}$ defined by the degree function $d$. Notice that in $\hat{A}^{\prime}$ the following relation holds:

$$
\hat{x}^{m} \hat{u}+\hat{y}^{k}+\hat{z}^{l}=0 .
$$

With this notation we have the following lemma.

Lemma 3.18. $\operatorname{Dk}_{\mathrm{gr}}\left(\hat{A}^{\prime}\right) \subset \hat{A}_{\leq 0}^{\prime}$, and so (by 1.24) $\mathrm{Dk}\left(A^{\prime}\right) \subset A_{0}^{\prime} \neq A^{\prime}$. Thereby $A^{\prime} \nsucceq \mathbb{C}^{[3]}$ and $X \not \subset \mathbb{C}^{3}$, which yields 3.15 .

Proof. We must show that $\operatorname{ker} \hat{\partial} \subset \hat{A}^{\prime} \leq 0$ whenever $\hat{\partial} \in \operatorname{LND}_{\mathrm{gr}}\left(\hat{A}^{\prime}\right)$. Assume that there exist $\hat{\partial} \in \operatorname{LND}_{\text {gr }}\left(\hat{A}^{\prime}\right)$ and $\hat{a} \in \operatorname{ker} \hat{\partial}$ such that $\hat{a} \notin \hat{A}^{\prime} \leq 0$. Furthermore, by 1.14(c) we may suppose that this element $\hat{a}$ is non-decomposable. We have $\hat{a}=\left.q\right|_{\hat{X}}$ for some $d$-homogeneous polynomial $q=\sum_{i, j} a_{i j} x^{i} u^{j} \in \mathbb{C}^{[4]}$ (with $a_{i j} \in \mathbb{C}[y, z]$ ). Moreover, taking into account (30) we may suppose that $i<m$ whenever $j>0$.

Claim. In the above expression for $q$ there is only one non-zero monomial.

Proof of the Claim. Indeed, if there were two of them, say, if $a_{i_{1} j_{1}} \neq 0$ and $a_{i_{2} j_{2}} \neq 0$, then we would have

$$
d(q)=m j_{1}-i_{1}=m j_{2}-i_{2} \quad \Longrightarrow \quad m\left(j_{1}-j_{2}\right)=i_{1}-i_{2} .
$$

Assuming that $i_{1}>i_{2}$, by (31) we get $j_{1}>j_{2}$, and vice versa. Thus $j_{1}>0$, and then by our assumption $i_{1}<m$. Hence also $i_{1}-i_{2}<m$, which contradicts (31). This proves the claim.

Since the element $\hat{a}=q(\hat{x}, \hat{y}, \hat{z}, \hat{u})=a_{i j}(\hat{y}, \hat{z}) \hat{x}^{i} \hat{u}^{j}$ is supposed to be nondecomposable and of positive $d$-degree, we have $\hat{a}=\hat{u} \in \operatorname{ker} \hat{\partial}$. Thus $\hat{\partial}$ can 
be specified to a locally nilpotent derivation $\partial_{1}$ of the algebra $B=\mathbb{C}[S]$, where $S:=\left\{x^{m}+y^{k}+z^{l}=0\right\}=\{u=1\} \cap \hat{X} \subset \mathbb{C}^{3}$ (see 1.16).

By $1.14(\mathrm{a}), \operatorname{tr} \cdot \operatorname{deg}(\operatorname{ker} \hat{\partial})=2$, hence there is a homogeneous $\hat{\partial}$-constant $\hat{b}$ such that the elements $\hat{a}=\hat{u}, \hat{b} \in \hat{A}^{\prime}$ are algebraically independent. As above we obtain that either $\hat{b}=b(\hat{y}, \hat{z})$ for some irreducible polynomial $b \in \mathbb{C}^{[2]} \backslash \mathbb{C}$, or $\hat{b}=\hat{x}$. In the latter case by (30) we have $\hat{y}^{k}+\hat{z}^{l} \in \operatorname{ker} \hat{\partial}$, and thus by 1.14(d) also $\hat{y}, \hat{z} \in \operatorname{ker} \hat{\partial}$. Therefore $\hat{\partial}=0$, which is impossible. Finally, we conclude that $b(\hat{y}, \hat{z}) \in \operatorname{ker} \hat{\partial}$ for a certain polynomial $b \in \mathbb{C}^{[2]} \backslash \mathbb{C}$, and so the restriction $\left.b\right|_{S}$ is a $\partial_{1}$-constant.

Now the proof can be completed by applying the next lemma (cf. [5, 20, 43]).

Lemma 3.19. Let $B=\mathbb{C}[S]$, where $S:=\left\{x^{m}+y^{k}+z^{l}=0\right\} \subset \mathbb{C}^{3}$ and $k, l, m \geq$ $2, \operatorname{gcd}(k, l)=1$, and let $\partial_{1} \in \operatorname{LND}(B)$. Then $\left.b\right|_{S} \notin \operatorname{ker} \partial_{1}$ whenever $b \in \mathbb{C}[y, z] \backslash \mathbb{C}$.

Proof. We define the weight degree function $\hat{d}$ on the algebra $B$ by letting

$$
\hat{d}_{x}=k l, \quad \hat{d}_{y}=l m, \quad \hat{d}_{z}=k m .
$$

Actually $x^{m}+y^{k}+z^{l}$ is a $\hat{d}$-homogeneous polynomial, $B$ is a graded algebra, and we may consider the associated homogeneous derivation $\hat{\partial}_{1} \in \operatorname{LND}_{\mathrm{gr}}(B)$. Assuming that for a polynomial $b \in \mathbb{C}[y, z] \backslash \mathbb{C},\left.b\right|_{S} \in \operatorname{ker} \partial_{1}$ we will get a $\hat{d}$-homogeneous polynomial $\hat{b}_{1} \in \mathbb{C}[y, z] \backslash \mathbb{C}$ such that $\left.\hat{b}_{1}\right|_{S} \in \operatorname{ker} \hat{\partial}_{1}$ as well. By 1.14 (c) an irreducible $\hat{d}$-homogeneous factor of the polynomial $\hat{b}_{1}$ (this can be $y, z$ or $\lambda y^{k}+\mu z^{l}$, where $\left.\lambda, \mu \in \mathbb{C}^{*}\right)$ restricts to $S$ as a $\hat{\partial}_{1}$-constant. If it were $\lambda y^{k}+\mu z^{l}$ then by 1.14(d) both $\left.y\right|_{S}$ and $\left.z\right|_{S}$ would be $\hat{\partial}_{1}$-constants, whence $\hat{\partial}_{1}=0$, a contradiction.

Thus we may assume that, say, $\left.y\right|_{S} \in \operatorname{ker} \hat{\partial}_{1}$. As $\left.\left(x^{m}+y^{k}+z^{l}\right)\right|_{S}=0$ we have $\left.\left(x^{m}+z^{l}\right)\right|_{S} \in \operatorname{ker} \hat{\partial}_{1}$, and (since $m, l \geq 2$ ) again by 1.14ld) we obtain that $\left.x\right|_{S},\left.z\right|_{S} \in \operatorname{ker} \hat{\partial}_{1}$, which is impossible.

This completes the proof of 3.193 .18 and 3.15

Remark 3.20. Choosing a weight degree function likewise in 3.11 and repeating the proof of Proposition 5.3 in 14 it can be shown that under assumptions as in 3.6 (that is, as in 3.9 or 3.15 one has $\mathbb{C}[x] \subseteq \mathrm{ML}\left(A^{\prime}\right)$, hence the Makar-Limanov invariant $\mathrm{ML}\left(A^{\prime}\right)$ is non-trivial as well.

3.2. Simple affine modifications of $\mathbb{C}^{3}$ isomorphic to $\mathbb{C}^{3}$. The following theorem is a central result of the paper. The expression 'in appropriate $(x, y)$-coordinates' below means 'after performing an appropriate automorphism $\alpha \in \operatorname{Aut} \mathbb{C}[x, y]$ $\subset$ Aut $\mathbb{C}[x, y, z, u]^{\prime}$. (In fact, the new $(x, y)$-coordinates are chosen in a way to get $f_{\text {non-horiz }} \in \mathbb{C}[x]$; in particular, no coordinate change is necessary when $f_{\text {non-horiz }}=$ const.)

Theorem 3.21. For a hypersurface $X \subset \mathbb{C}^{4}$ given by an equation

$$
p=f(x, y) u+g(x, y, z)=0 \quad\left(f \in \mathbb{C}^{[2]} \backslash\{0\}, g \in \mathbb{C}^{[3]}\right),
$$

in appropriately chosen new $(x, y)$-coordinates the following conditions (i)-(vi) are equivalent:

(i) $X \simeq \mathbb{C}^{3}$.

(ii) Every fiber $X_{\mu}=p^{-1}(\mu)(\mu \in \mathbb{C})$ of the polynomial $p \in \mathbb{C}^{[4]}$ is isomorphic to $\mathbb{C}^{3}$. 
(iii) Every fiber of the regular function $\left.x\right|_{X} \in A^{\prime}:=\mathbb{C}[X]$ is reduced and isomorphic to $\mathbb{C}^{2}$.

(iv) Every fiber of the morphism $\rho: \mathbb{C}^{4} \rightarrow \mathbb{C}^{2},(x, y, z, u) \longmapsto(x, p(x, y, z, u))$, is reduced and isomorphic to $\mathbb{C}^{2}$.

(v) The polynomial $p$ is an $x$-residual variable 12

(vi) With $Y:=\mathbb{C}^{3}$ and $X \subseteq \mathbb{C}^{4}$ as above being smooth and irreducible, conditions $2.11(\alpha)-(\delta)$ hold as well as the following:

$(\varepsilon)$ The curves $C_{\text {non-horiz }}$ and $\Gamma_{\text {non-horiz }}$ are smooth.

Proof. The implications (v) $\Rightarrow$ (iv) $\Rightarrow$ (iii) and (ii) $\Rightarrow$ (i) are immediate; (i) $\Rightarrow$ (vi) follows from 2.28 and 3.6. Thus it suffices to establish (vi) $\Rightarrow(\mathrm{v})$ and (iii) $\Rightarrow$ (ii).

Let us show (vi) $\Rightarrow(\mathrm{v})$. If $f_{\text {non-horiz }} \neq$ const (that is, $h<n$ ), then by our assumptions 2.11 $(\gamma)$ and $(\mathrm{vi})(\varepsilon), \Gamma_{h+1} \simeq \mathbb{C}$. By the Abhyankar-Moh-Suzuki Theorem, after performing an appropriate automorphism of the plane $\mathbb{C}_{x, y}^{2}$ we may suppose that $f_{h+1}=x$. Hence by $2.11(\gamma)$ we have

$$
f_{\text {non-horiz }}=c \prod_{i=h+1}^{n}\left(x-\lambda_{i}\right)^{a_{i}} \in \mathbb{C}[x] .
$$

Anyhow, $f_{\text {non-horiz }}=$ const or not, assuming that $f_{\text {horiz }} \neq$ const by 3.4 we may write

$$
p_{\lambda}=\kappa f_{\text {horiz }, \lambda} u+b_{0, \lambda}+b_{1, \lambda} z+f_{\text {horiz }, \lambda}^{\text {red }} h_{\lambda}
$$

with $f_{\text {horiz }, \lambda}, f_{\text {horiz }, \lambda}^{\text {red }}, b_{0, \lambda}, b_{1, \lambda} \in B:=\mathbb{C}[y], h_{\lambda} \in B[z]$ and $\kappa:=f_{\text {non-horiz }}(\lambda)=$ $c \prod_{i=h+1}^{n}\left(\lambda-\lambda_{i}\right)^{a_{i}} \in \mathbb{C}$. If $\lambda \in \mathbb{C} \backslash\left\{\lambda_{h+1}, \ldots, \lambda_{n}\right\}$ (i.e., $\kappa \neq 0$ ), then by $3.4 b_{1, \lambda} \in B$ is invertible $\bmod f_{\text {horiz }, \lambda}$, whereas $f_{\text {horiz }, \lambda}^{\text {red }}$ is nilpotent $\bmod f_{\text {horiz }, \lambda}$. Therefore by [1.29, $p_{\lambda} \in B[z, u]$ is a $B$-variable; in particular, $p_{\lambda} \in \mathbb{C}[y, z, u]$ is a variable (hence for every $\mu \in \mathbb{C}$, the surface $\left\{p_{\lambda}=\mu\right\} \subseteq \mathbb{C}_{y, z, u}^{3}$ is reduced and isomorphic to $\mathbb{C}^{2}$ ).

For every $i=h+1, \ldots, n$ we have $f_{i}=x-\lambda_{i}$ and, by 2.11( $(\gamma)$ and (vi) $(\varepsilon)$, $\Gamma_{i} \simeq \mathbb{C} \simeq C_{i}$. Moreover by 2.3(i) the polynomial $g_{\lambda_{i}} \in \mathbb{C}[y, z]$ is irreducible and $g_{\lambda_{i}}^{-1}(0)=C_{i} \simeq \mathbb{C}$, hence by the Abhyankar-Moh-Suzuki Theorem it is a variable of the polynomial ring $\mathbb{C}[y, z]$. Thus $p_{\lambda_{i}}=g_{\lambda_{i}} \in \mathbb{C}[y, z, u]$ is a variable as well. Now (v) follows. In the remaining case where $f_{\text {horiz }}=$ const the proof is similar (but simpler) and left to the reader.

Next we prove (iii) $\Rightarrow$ (ii). We notice first of all that under the condition (iii), the hypersurface $X$ is smooth and irreducible (indeed, for every $\lambda \in \mathbb{C}$ we have

$$
\left.\operatorname{grad} p_{\lambda}=\operatorname{pr}\left((\operatorname{grad} p)_{\lambda}\right) \quad \text { with } \quad \operatorname{pr}:(x, y, z, u) \longmapsto(y, z, u)\right) .
$$

Similarly, under condition (iv) every fiber $X_{\mu}(\mu \in \mathbb{C})$ is smooth and irreducible.

Actually we establish (iii) $\Rightarrow$ (i), which at the same time shows (iv) $\Rightarrow$ (ii). Together with the implication (i) $\Rightarrow$ (iv) (which we already know) these yield (iii) $\Rightarrow$ (ii), as needed.

To prove the implication (iii) $\Rightarrow(\mathrm{i})$, by 1.7 it is enough to show that a smooth, irreducible 3-fold $X$ satisfying (iii) is acyclic. Let $U=\mathbb{C} \backslash V$ be a Zariski open subset such that over $U$, the morphism $\left.x\right|_{X}: X \rightarrow \mathbb{C}$ is a smooth fibration (with

\footnotetext{
${ }^{12}$ That is 1.27 for any $\lambda \in \mathbb{C}$, the specialization $p_{\lambda} \in \mathbb{C}[y, z, u]$ of $p$ is a variable.
} 
the fibers diffeomorphic to $\mathbb{R}^{4}$ ). In the notation of 1.9 we let $\hat{X}=X, \hat{Y}=Y=$ $\mathbb{C}^{3}, \hat{E}=\left(\left.x\right|_{X}\right)^{-1}(V) \subseteq \hat{X}$ and $\hat{D}=x^{-1}(V) \subseteq \hat{Y}$. It is easily seen that the induced homomorphisms

$$
\begin{aligned}
& \left(\left.x\right|_{\hat{X} \backslash \hat{E}}\right)_{*}: H_{*}(\hat{X} \backslash \hat{E}) \rightarrow H_{*}(\mathbb{C} \backslash V), \\
& \left(\left.x\right|_{\hat{Y} \backslash \hat{D}}\right)_{*}: H_{*}(\hat{Y} \backslash \hat{D}) \rightarrow H_{*}(\mathbb{C} \backslash V),
\end{aligned}
$$

and hence also

$$
\left(\left.\sigma\right|_{\hat{X} \backslash \hat{E}}\right)_{*}: H_{*}(\hat{X} \backslash \hat{E}) \rightarrow H_{*}(\hat{Y} \backslash \hat{D})
$$

are isomorphisms, as well as

$$
\left(\left.\sigma\right|_{\hat{E}}\right)_{*}: H_{*}(\hat{E}) \rightarrow H_{*}(\hat{D})
$$

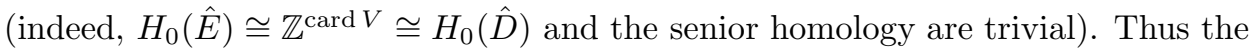
conditions (i) and (ii) of 1.9 are fulfilled, and so by 1.9

$$
\sigma_{*}: H_{*}(\hat{X}) \rightarrow H_{*}(\hat{Y})
$$

is an isomorphism. Therefore, the 3 -fold $X=\hat{X}$ is acyclic. This completes the proof.

Remark 3.22. For another proof of the implication (iii) $\Rightarrow$ (i) which do not use 1.7 see 4.25, 4.26 below.

3.23. If the polynomial $p \in \mathbb{C}^{[4]}$ is linear with respect to two (and not just one) variables we have a much stronger result (see 3.24 below). It is an analog in dimension 4 of Theorem 7.2 in [19] generalizing Sathaye's Theorem [34]. For a polynomial $\varphi \in \mathbb{C}[x, y]$ we let $\Gamma_{\varphi}:=V(\varphi) \subseteq \mathbb{C}^{2}$. Also, we use the variable $v$ below instead of $z$ to emphasize the symmetry of the situation.

Theorem 3.24. For a hypersurface $X \subset \mathbb{C}^{4}$ given by an equation

$$
p=\tilde{a}(x, y) u+\tilde{b}(x, y) v+c(x, y)=d(a u+b v)+c=0
$$

with $a, b, c, d \in \mathbb{C}[x, y], d \neq 0,(a, b) \neq(0,0)$ and $\operatorname{gcd}(a, b)=1$, in appropriately chosen new $(x, y)$-coordinates the following conditions $\left(\mathrm{i}^{\prime}\right)-\left(\mathrm{v}^{\prime}\right)$ are equivalent:

$\left(\mathrm{i}^{\prime}\right)$ The 3 -fold $X$ is irreducible, smooth and acyclic.

(ii') $X \simeq \mathbb{C}^{3}$.

(iii') The polynomial $p \in \mathbb{C}^{[4]}$ is an $x$-residual variable.

$\left(\mathrm{iv}^{\prime}\right) p \in \mathbb{C}[x]^{[3]}$ is an $x$-variable.

$\left(\mathrm{v}^{\prime}\right) d \in \mathbb{C}[x], \operatorname{gcd}(c, d)=1, \Gamma_{a} \cap \Gamma_{b} \subseteq \Gamma_{d}$, and for every root $x=x_{i}$ of $d(i=1, \ldots, \operatorname{deg} d)$ we have

$$
c_{x_{i}}(y)=c\left(x_{i}, y\right) \in \mathbb{C}^{*} y+\mathbb{C} .
$$

Proof. The strategy of the proof is as follows. By[3.21 the conditions (ii') and (iii') are equivalent to each other and to the other conditions of 3.21 . The equivalence $\left(\mathrm{iii}^{\prime}\right) \Longleftrightarrow\left(\mathrm{iv}^{\prime}\right)$ will be established later on in $4.2(\mathrm{~b})$. By[2.11 we have $\left(\mathrm{i}^{\prime}\right) \Longrightarrow 2.11(\alpha)$ $(\delta)$. We show below that $\left(\mathrm{i}^{\prime}\right)$ also implies condition $(\varepsilon)$ of 3.21 (vi). By 3.21 this yields the implications $\left(\mathrm{i}^{\prime}\right) \Longrightarrow 3.21(\mathrm{vi}) \Longleftrightarrow\left(\mathrm{ii}^{\prime}\right) \Longrightarrow\left(\mathrm{i}^{\prime}\right)$, and so gives $\left(\mathrm{i}^{\prime}\right) \Longleftrightarrow\left(\mathrm{ii}^{\prime}\right)$. Finally we show $\left(\mathrm{iii}^{\prime}\right) \Longleftrightarrow\left(\mathrm{v}^{\prime}\right)$, which concludes the proof. 
Interchanging (if necessary) the roles of $u$ and $v$ we may suppose in the sequel that $a \neq 0$.

$\left(\mathrm{i}^{\prime}\right) \Longrightarrow(\varepsilon)$. We observe (see 3.3) that for $p$ as in (32), $C_{\text {slant }}=\Gamma_{\text {slant }}=\emptyset$, hence $C_{\text {non-horiz }}=C_{\text {vert }}$ is smooth. It was shown in the proof of 3.10 that if $\Gamma_{\text {non-horiz }}$ is singular, then $\Gamma_{\text {non-horiz }}=\Gamma_{\text {slant }}$. Henceforth in our setting $\Gamma_{\text {non-horiz }}$ is also smooth, which shows $(\varepsilon)$.

$\left(\right.$ iii $\left.^{\prime}\right) \Longrightarrow\left(\mathrm{v}^{\prime}\right)$. As $X$ is irreducible we have $\operatorname{gcd}(c, d)=1$. Letting $q:=p-c$ we fix a point $P=\left(x_{0}, y_{0}\right)$ such that

$$
q_{P}=d\left(x_{0}, y_{0}\right)\left(a\left(x_{0}, y_{0}\right) u+b\left(x_{0}, y_{0}\right) v\right)=0 .
$$

It is easily seen that $y-y_{0}$ divides $p_{x_{0}}-c\left(x_{0}, y_{0}\right)=q_{x_{0}}+\left(c_{x_{0}}-c\left(x_{0}, y_{0}\right)\right)$. As $p_{x_{0}} \in$ $\operatorname{Var}_{\mathbb{C}}[y, u, v]$ we obtain that $q_{x_{0}}=0$ and $p_{x_{0}}-c\left(x_{0}, y_{0}\right)=c_{x_{0}}-c\left(x_{0}, y_{0}\right)=\kappa\left(y-y_{0}\right)$ with $\kappa \in \mathbb{C}^{*}$, as needed in (33). As $\operatorname{gcd}(a, b)=1$, from $q_{x_{0}}=0$ we get $d_{x_{0}}=0$. Thus for every root $\left(x_{0}, y_{0}\right)$ of $d=0$ we have $d_{x_{0}}=0$, which means that $d \in \mathbb{C}[x]$. Moreover, for every root $\left(x_{0}, y_{0}\right)$ of $a=b=0$ we also have $d_{x_{0}}=0$, which shows the inclusion $\Gamma_{a} \cap \Gamma_{b} \subseteq \Gamma_{d}$ and gives $\left(\mathrm{v}^{\prime}\right)$.

$\left(\mathrm{v}^{\prime}\right) \Longrightarrow\left(\right.$ iii'). In view of (33) for every root $x_{i}$ of $d(i=h+1, \ldots, n), p_{x_{i}}=$ $c_{x_{i}} \in \operatorname{Var}_{\mathbb{C}} \mathbb{C}[y] \subseteq \operatorname{Var}_{\mathbb{C}} \mathbb{C}[y, u, v]$. Furthermore let $\lambda \in \mathbb{C}$ and $d(\lambda) \neq 0$. As by $\left(\mathrm{v}^{\prime}\right), \Gamma_{a} \cap \Gamma_{b} \subseteq \Gamma_{d}$ we have $\operatorname{gcd}\left(a_{\lambda}, b_{\lambda}\right)=1$, whence $r_{\lambda} a_{\lambda}-s_{\lambda} b_{\lambda}=1$ for certain polynomials $r_{\lambda}, s_{\lambda} \in \mathbb{C}[y]$. As the matrix

$$
A_{\lambda}=\left(\begin{array}{cc}
a_{\lambda} & b_{\lambda} \\
s_{\lambda} & r_{\lambda}
\end{array}\right)
$$

is invertible over the ring $\mathbb{C}[y]$, the mapping

$$
\alpha:(u, v) \longmapsto\left(u_{1}, v_{1}\right):=A_{\lambda}(u, v)
$$

induces a $\mathbb{C}[y]$-automorphism of $\mathbb{C}[y][u, v]$ with $\alpha\left(p_{\lambda}\right)=d(\lambda) u_{1}+c_{\lambda} \in \operatorname{Var}_{\mathbb{C}} \mathbb{C}[y, u, v]$. Thus (iii') follows.

\section{Simple birational extensions of $\mathbb{C}^{[3]}$ AS VARiables in $\mathbb{C}^{[4]}$}

4.1. Partial positive results. We recall the problem stated in the Introduction.

4.1. Problem. Is it true that if a hypersurface $X \subseteq \mathbb{C}^{4}$ with equation of the form

$$
p:=f(x, y) u+g(x, y, z)=0 \quad \text { (where } \quad f \in \mathbb{C}^{[2]} \backslash\{0\} \quad \text { and } \quad g \in \mathbb{C}^{[3]} \text { ) }
$$

is isomorphic to $\mathbb{C}^{3}$, then necessarily $p \in \operatorname{Var}_{\mathbb{C}} \mathbb{C}^{[4]}$ and moreover, $p \in \operatorname{Var}_{B} B^{[3]}$ (that is, $p$ is an $x$-variable) provided in addition that $f_{\text {non-horiz }} \in B:=\mathbb{C}[x]$ ?

The principal results of this subsection can be summarized as follows.

Theorem 4.2. The answer to 4.1 is positive in each of the following cases:

(a) $f \in \mathbb{C}[x]$.

(b) $\operatorname{deg}_{z} g \leq 1$.

(c) $f_{\text {vert }}=1$.

(d) $f_{\text {horiz }}=f_{\text {horiz }}^{\text {red }}$.

(e) $g$ is of form $g=g_{0}(x, y)+\check{g}_{2}(x, y, z) z^{2}$.

The proof is given in 4.15 below. From $4.2(\mathrm{c})$, (d) we obtain the following corollary.

Corollary 4.3. If in 4.1. $f=f_{1}^{a_{1}}$ with $f_{1} \in \mathbb{C}[x, y]$ irreducible, then $p$ is a variable. 
4.4. By 3.21(v) we may suppose that $p$ as in 4.1 is an $x$-residual variable (see 1.25 1.27). Thus 4.1 would be answered in the positive if the following conjecture 13 were true.

Conjecture 4.5. If $p \in \mathbb{C}^{[n]}$ is a residual $x_{1}$-variable (that is,

$$
\left.p_{\lambda}:=p\left(\lambda, x_{2}, \ldots, x_{n}\right) \in \operatorname{Var}_{\mathbb{C}} \mathbb{C}^{[n-1]} \quad \forall \lambda \in \mathbb{C}\right),
$$

then $p$ is an $x_{1}$-variable: $p \in \operatorname{Var}_{\mathbb{C}\left[x_{1}\right]} \mathbb{C}\left[x_{1}\right]^{[n-1]}$.

In 4.614.8 below we analyze the situation with the only assumption that $p=$ $f u+g$ is an $x$-residual variable. First in 4.6 we include the case where $f=0$, then in 4.7 we deal with the special case where $f \in \mathbb{C}[x] \backslash\{0\}$, whereas the general case is treated in 4.8 .

Proposition 4.6. Let $X$ be a smooth affine surface and $\varphi: X \rightarrow \mathbb{C}$ be a morphism with $\varphi^{*}(\lambda) \simeq \mathbb{C} \forall \lambda \in \mathbb{C}$. Then the following hold:

(a) $X \simeq \mathbb{C}^{2}$.

(b) Furthermore, if $X=\operatorname{spec}\left(\mathbb{C}^{[3]} /(g)\right)$ with $g \in \mathbb{C}^{[3]}=\mathbb{C}[x, y, z]$ and $\varphi=\left.x\right|_{X}$, then $g$ is an $x$-variable.

(c) Consequently, an $x$-residual variable $g \in \mathbb{C}[x, y, z]$ is an $x$-variable ${ }^{14}$

Proof. (a) The fact is well-known (e.g., cf. [30, Thm. 2.2.1]); nevertheless we indicate the proof. First extending $\varphi: X \rightarrow \mathbb{C}$ to a projective morphism $\bar{\varphi}: V \rightarrow \mathbb{P}^{1}$ of a smooth ruled surface $V$, we then pass to a smooth relatively minimal model of $(V, \bar{\varphi})$ by contracting successively the superfluous components of the reducible fibers of $\bar{\varphi}$ different from the closures of the original fibers of $\varphi$ (this is always possible; see, e.g., [12, Lemma 7], [42, Lemma 3.5] or [30, Lemma 1.4.1(6)]). Thus we obtain (as a completion of the original family) a Hirzebruch surface $\pi: \Sigma_{n} \rightarrow \mathbb{P}^{1}$ with a section $s: \mathbb{P}^{1} \rightarrow \Sigma_{n}$ 'at infinity', so that $X=\Sigma_{n} \backslash\left(s\left(\mathbb{P}^{1}\right) \cup F_{\infty}\right)$. By means of elementary transformations over the point $\infty \in \mathbb{P}^{1}$ we replace $\Sigma_{n}$ with $\Sigma_{0}=\mathbb{P}^{1} \times \mathbb{P}^{1}$ and $s\left(\mathbb{P}^{1}\right)$ with a constant section, say, $C_{0}$. This yields the desired isomorphism $X=\Sigma_{0} \backslash\left(C_{0} \cup F_{\infty}\right) \simeq \mathbb{C}^{2}$.

(b) By (a) there is an isomorphism

$$
\gamma: \mathbb{C}[x, y, z] /(g) \rightarrow \mathbb{C}^{[2]}=\mathbb{C}[x, y] .
$$

Letting $h:=\gamma(x) \in \mathbb{C}^{[2]}$, by our assumption we obtain

$$
\mathbb{C}^{[2]} /(h)=\mathbb{C}[x, y, z] /(g, x)=\mathbb{C}\left[\varphi^{*}(0)\right] \cong \mathbb{C}^{[1]} .
$$

Hence by the Abhyankar-Moh-Suzuki Theorem, up to an automorphism of $\mathbb{C}[x, y]$ we may suppose that $h=x$, i.e.,

$$
\gamma: \mathbb{C}[x][y, z] /(g) \rightarrow \mathbb{C}[x]^{[1]} .
$$

Now it follows from the generalized version of the Abhyankar-Moh-Suzuki Theorem 33, Thm. 2.6.2] that $g$ is an $x$-variable, as stated.

(c) immediately follows from (a) and (b).

\footnotetext{
${ }^{13}$ Proposed by the second author.

${ }^{14}$ Moreover, $g \in \mathbb{C}[x, y, z]$ is an $x$-residual variable of the ring $\mathbb{C}[x][y, z, u]$ iff it is an $x$-variable. Indeed, this follows from (c) and the cancellation for curves: $\Gamma \times \mathbb{C} \simeq \mathbb{C}^{2} \Longrightarrow \Gamma \simeq \mathbb{C}$.
} 
Proposition 4.7. If $p=f(x) u+g(x, y, z) \in \mathbb{C}^{[4]}$ (with $f \in \mathbb{C}[x] \backslash\{0\}$ ) is an $x$-residual variable, then actually it is an $x$-variable.

Proof. As $u+g(x, y, z)$ is an $x$-variable, by 1.31(a) so is $p=f u+g$.

Proposition 4.8. If $p=f(x, y) u+g(x, y, z) \in \mathbb{C}[x][y, z, u]$ with $f \notin \mathbb{C}[x]$ is an $x$-residual variable, then $p$ can be written as follows:

$$
p=q\left[r \tilde{f} u+\tilde{g}_{0} y^{2}+\tilde{g}_{1} z+\tilde{f}^{\mathrm{red}} \tilde{g}_{2} z^{2}\right]+a_{0}+a_{1} y,
$$

where

- $\quad q, r, a_{0}, a_{1} \in \mathbb{C}[x], \tilde{f}, \tilde{g}_{0}, \tilde{g}_{1} \in \mathbb{C}[x, y], \quad \tilde{g}_{2} \in \mathbb{C}[x, y, z]$,

- $\tilde{f}^{-1}(0) \cap \tilde{g}_{1}^{-1}(0)$ is a finite subset of $q^{-1}(0) \times \mathbb{C}_{y}$, and

- for every root $x_{0}$ of $r, q\left(x_{0}\right) \tilde{f}\left(x_{0}, y\right) \in \mathbb{C}$.

The proof is done in 4.11 below.

Corollary 4.9. If $p=f(x, y) u+g(x, y, z) \in \mathbb{C}[x][y, z, u]$ is an $x$-residual variable, then it is a $\mathbb{C}(x)$-variable of $\mathbb{C}(x)^{[3]}$.

Proof. As a $\mathbb{C}[x]$-variable is also a $\mathbb{C}(x)$-variable, by 4.6 and 4.7 we may suppose that $f \notin \mathbb{C}[x]$, and so 4.8 applies. Let $p$ be presented as in (34). As $\tilde{f}^{-1}(0) \cap \tilde{g}_{1}^{-1}(0)$ is finite, the polynomials $q r \tilde{f}, q \tilde{g}_{1} \in \mathbb{C}[x, y]$ regarded as elements of $B:=\mathbb{C}(x)[y]$ are coprime. Moreover the polynomial $\tilde{f}^{\text {red }} \tilde{g}_{2} z^{2} \in B[z]$ is nilpotent $\bmod (q r \tilde{f})$, and so by 1.29 (a), $p=f u+g \in \operatorname{Var}_{B} B[z, u]$.

The proof of 4.8 relies on the following lemma (cf. [34 19 39]).

Lemma 4.10. Let $p=f(y) u+g(y, z)$ be a variable of $\mathbb{C}[y, z, u]$.

(a) If $f \neq 0$, then $p$ is an $y$-variable, and it can be written as follows:

$$
p=f(y) u+g_{0}(y)+g_{1}(y) z+f^{\mathrm{red}}(y) \tilde{g}_{2}(y, z) z^{2}
$$

with $\operatorname{gcd}\left(f, g_{1}\right)=1$.

(b) If $f=0$, then the coefficient of the highest order term in $z$ of $p$ (which a priori is a polynomial in $y$ ) is constant, unless $\operatorname{deg}_{z} g \leq 0$.

Proof. (a) The statement is evidently true if $f=$ const. Suppose that $f \notin \mathbb{C}$. By our assumption $\mathbb{C}^{[3]} /(p) \cong \mathbb{C}^{[2]}$. If $y_{1} \in \mathbb{C}$ is such that $f\left(y_{1}\right)=1$, then we have

$$
\mathbb{C}[y, z, u] /\left(f(y) u+g(y, z), y-y_{1}\right) \cong \mathbb{C}[z, u] /\left(u+g_{y_{1}}(z)\right) \cong \mathbb{C}^{[1]} .
$$

By the Abhyankar-Moh-Suzuki Theorem, the same is true for every value of $y$. In particular, for any root $y_{0}$ of $f$ we have

$$
\mathbb{C}[y, z, u] /\left(f(y) u+g(y, z), y-y_{0}\right) \cong \mathbb{C}[z, u] /\left(g_{y_{0}}(z)\right) \cong \mathbb{C}^{[1]} .
$$

Therefore $\operatorname{deg}_{z} g_{y_{0}}(z)=1, p=f u+g$ has the desired form, and by 1.29 (a) (with $B:=\mathbb{C}[y])$ it is a $y$-variable.

(b) In the course of the proof of 1.31 we have noticed that the polynomials of $\mathbb{C}[y, z]$ which are variables of $\mathbb{C}[y, z, u]$ are actually variables of $\mathbb{C}[y, z]$. Thus $g \in \operatorname{Var}_{\mathbb{C}} \mathbb{C}[y, z]$, and so statement (b) is well known (e.g., see [1]). 
4.11. Proof of Proposition 4.8. It is easily seen that the polynomial $p=f u+g \in$ $\mathbb{C}[x][y, z, u]$ admits a presentation

$$
p=q\left[\bar{f} u+\tilde{g}_{0} y^{2}+\tilde{g}_{1} z+\bar{g}_{2} z^{2}\right]+a_{0}+a_{1} y
$$

with $a_{0}, a_{1} \in \mathbb{C}[x], \bar{f}, \tilde{g}_{0}, \tilde{g}_{1} \in \mathbb{C}[x, y], \bar{g}_{2} \in \mathbb{C}[x, y, z]$ and with $q \in \mathbb{C}[x]$ being a monic polynomial of maximal degree such that (35) holds. Clearly,

$$
q^{-1}(0)=L(p):=\left\{x_{0} \in \mathbb{C} \mid p_{x_{0}}=f\left(x_{0}, y\right) u+g\left(x_{0}, y, z\right) \in \mathbb{C} y+\mathbb{C}\right\}
$$

(possibly, this set is empty, and then we put $q:=1$ ). Since, by our assumption, $f \notin \mathbb{C}[x]$, the subset

$$
K=K(f):=\left\{\lambda \in \mathbb{C} \mid f_{\lambda}(y) \in \mathbb{C}\right\}=q^{-1}(0) \cup\left\{\lambda \in \mathbb{C} \mid \bar{f}_{\lambda}(y) \in \mathbb{C}\right\} \subseteq \mathbb{C}
$$

is finite. We let $x_{0} \in \mathbb{C} \backslash K(f)$. The specialization $p_{x_{0}}$ being a variable of $\mathbb{C}[y, z, u]$, by 4.10 (a) we have $\operatorname{gcd}\left(\bar{f}\left(x_{0}, y\right), \tilde{g}_{1}\left(x_{0}, y\right)\right)=1$, hence $\bar{f}^{-1}(0) \cap \tilde{g}_{1}^{-1}(0) \subseteq K \times \mathbb{C}_{y}$.

Since by $4.10(\mathrm{a}), p_{x_{0}} \in \mathbb{C}[y, z, u]$ is a residual $y$-variable, the equality $\bar{f}\left(x_{0}, y_{0}\right)=$ 0 implies that $\operatorname{deg}_{z} g_{x_{0}, y_{0}}(z)=1$, hence also $\bar{g}_{2}\left(x_{0}, y_{0}, z\right)=0$. It follows that

$$
\bar{f}^{-1}(0) \backslash\left(K \times \mathbb{C}_{y}\right) \subseteq \bar{g}_{2}^{-1}(0) .
$$

It is easily seen that $\bar{f} \in \mathbb{C}[x, y]$ admits a factorization $\bar{f}(x, y)=r(x) \tilde{f}(x, y)$ such that $r^{-1}(0) \subseteq K$ and $\tilde{f}^{-1}(0) \cap\left(K \times \mathbb{C}_{y}\right)$ is finite. Then we have

$$
\tilde{f}^{-1}(0) \backslash\left(K \times \mathbb{C}_{y}\right) \subseteq \bar{g}_{2}^{-1}(0) .
$$

Passing to the Zariski closures we obtain

$$
\tilde{f}^{-1}(0) \subseteq \bar{g}_{2}^{-1}(0), \quad \text { i.e., } \quad \bar{g}_{2}=\tilde{f}^{\text {red }} \tilde{g}_{2} .
$$

Now (35) yields a desired presentation (34). As $r^{-1}(0) \subseteq K(f)$, by the definition of $K(f)$ for every root $x_{0}$ of $r$ we have $q\left(x_{0}\right) \tilde{f}\left(x_{0}, y\right) \in \mathbb{C}$, and so it remains to check that $\tilde{f}^{-1}(0) \cap \tilde{g}_{1}^{-1}(0)$ is a finite subset of $q^{-1}(0) \times \mathbb{C}_{y}$.

We already know that $\tilde{f}^{-1}(0) \cap \tilde{g}_{1}^{-1}(0)$ is a finite subset of $K \times \mathbb{C}_{y}$. Suppose that there is a point

$$
\left(x_{0}, y_{0}\right) \in\left[\tilde{f}^{-1}(0) \cap \tilde{g}_{1}^{-1}(0)\right] \backslash q^{-1}(0) .
$$

Then $x_{0} \in K(f) \backslash q^{-1}(0)$, hence $\bar{f}\left(x_{0}, y\right) \in \mathbb{C}$, and so

$$
\bar{f}\left(x_{0}, y\right)=\bar{f}\left(x_{0}, y_{0}\right)=r\left(x_{0}\right) \tilde{f}\left(x_{0}, y_{0}\right)=0 .
$$

By our assumption,

$$
p_{x_{0}}=q\left(x_{0}\right)\left[\tilde{g}_{0}\left(x_{0}, y\right) y^{2}+\tilde{g}_{1}\left(x_{0}, y\right) z+\tilde{f}^{\mathrm{red}}\left(x_{0}, y\right) \tilde{g}_{2}\left(x_{0}, y, z\right) z^{2}\right]+a_{0}\left(x_{0}\right)+a_{1}\left(x_{0}\right) y
$$

is a variable of $\mathbb{C}[y, z, u]$, and thus also of $\mathbb{C}[y, z]$. Hence by 4.10 b), $\tilde{f}^{\text {red }}\left(x_{0}, y\right)=$ $\tilde{f}^{\text {red }}\left(x_{0}, y_{0}\right)=0$, and then again by $4.10(\mathrm{~b}), \tilde{g}_{1}\left(x_{0}, y\right)=\tilde{g}_{1}\left(x_{0}, y_{0}\right)=0$. Therefore, $p_{x_{0}} \in \mathbb{C}[y]$ is a variable, hence $q\left(x_{0}\right)=0$, a contradiction.

Remark 4.12. In the notation of 4.8 if $p=f u+g=q r \tilde{f} u+g$ is an $x$-residual variable, then $q \tilde{f} u+g$ is also. Moreover, by 1.31(a), if $q \tilde{f} u+g$ is an $x$-variable, then $p=f u+g$ is so as well.

Lemma 4.13. Let $p=f u+g \in \mathbb{C}[x][y, z, u]$ be an $x$-residual variable presented as in (34). If $\tilde{f}^{-1}(0) \cap \tilde{g}_{1}^{-1}(0)=\emptyset$, then $p$ is an $x$-variable. 
Proof. By 4.12 we may put in (34) $r=1$. By 1.29(a), the polynomial

$$
\tilde{p}:=\tilde{f}(x, y) u+\tilde{g}_{0}(x, y) y^{2}+\tilde{g}_{1}(x, y) z+\tilde{f}^{\mathrm{red}}(x, y) \tilde{g}_{2}(x, y, z) z^{2}
$$

is an $(x, y)$-variable, i.e., there is an automorphism $\alpha \in \operatorname{Aut}_{B} B[z, u]$ (with $B:=$ $\mathbb{C}[x, y]$ ) such that $\alpha(u)=\tilde{p}$ (indeed by our assumption, $\tilde{g}_{1}$ is invertible $\bmod \tilde{f}$ in $B)$. Furthermore by our assumption, for every root $x_{0}$ of $q$ the specialization

$$
p_{x_{0}}=f\left(x_{0}, y\right) u+g\left(x_{0}, y, z\right)=a_{0}\left(x_{0}\right)+a_{1}\left(x_{0}\right) y \in \mathbb{C}[y, z, u]
$$

is a variable. Consequently, $a_{1}\left(x_{0}\right) \neq 0$, and $\operatorname{so~} \operatorname{gcd}\left(q, a_{1}\right)=1$. It follows that there is an affine automorphism $\beta \in \operatorname{Aut}_{\mathbb{C}[x]} \mathbb{C}[x][y, u]$ such that

$$
\beta(y)=q(x) u+a_{0}(x)+a_{1}(x) y .
$$

Now $\alpha \beta \in \operatorname{Aut}_{\mathbb{C}[x]} \mathbb{C}[x][y, z, u]$ is such that

$$
\alpha \beta(y)=q \tilde{p}+a_{0}(x)+a_{1}(x) y=f u+g=p,
$$

as desired.

Lemma 4.14. If $p=f(x, y) u+g(x, y, z)$ is an $x$-residual variable of degree at most 1 in $z$, then it is an $x$-variable.

Proof. It follows from 4.8 that if $f \notin \mathbb{C}[x]$, then in (34) $\tilde{g}_{1} \neq 0$ (indeed, otherwise $\tilde{f}^{-1}(0) \cap \tilde{g}_{1}^{-1}(0)$ cannot be a finite subset of $\left.q^{-1}(0) \times \mathbb{C}_{y}\right)$. Thus if $\operatorname{deg}_{z} g \leq 0$, then $f \in \mathbb{C}[x]$, and so the statement follows from 4.7 .

If $\operatorname{deg}_{z} g=1$, then by $4.8 p=f u+g$ can be written as follows:

$$
p=q(x)\left[r(x) \tilde{f}(x, y) u+\tilde{g}_{0}(x, y) y^{2}+\tilde{g}_{1}(x, y) z\right]+a_{0}(x)+a_{1}(x) y
$$

with $\tilde{f}^{-1}(0) \cap \tilde{g}_{1}^{-1}(0)$ being a finite subset of $q^{-1}(0) \times \mathbb{C}_{y}$. By 4.12 we may assume that $r=1$.

We proceed by induction on the intersection index $n:=\tilde{f}^{*}(0) \cdot \tilde{g}_{1}^{*}(0)$. If $n=0$, then the statement follows from 4.13 Suppose that $n \geq 1$.

Let $x_{0} \in q^{-1}(0)$ be a coordinate of an intersection point $\left(x_{0}, y_{0}\right) \in \tilde{f}^{-1}(0) \cap$ $\tilde{g}_{1}^{-1}(0)$. Up to a translation we may suppose that $x_{0}=0$. As

$$
\min \left\{\operatorname{deg}_{y} \tilde{g}_{1}(0, y), \operatorname{deg}_{y} \tilde{f}(0, y)\right\}>0
$$

we may write

$$
\tilde{g}_{1}(0, y) z+\tilde{f}(0, y) u=d(y)(a(y) z+b(y) u),
$$

where $\operatorname{gcd}(a, b)=1$. Hence there is a linear automorphism $\gamma \in \operatorname{Aut}_{B} B[z, u]$ (with $B:=\mathbb{C}[x, y])$ such that $\gamma(a(y) z+b(y) u)=z$. It is not difficult to verify that applying $\gamma$ amounts to

$$
\operatorname{Jac}(\gamma)\left[\begin{array}{c}
\tilde{g}_{1} \\
\tilde{f}
\end{array}\right]=\left[\begin{array}{l}
\hat{g}_{1} \\
x \hat{f}
\end{array}\right]
$$

for certain $\hat{f}, \hat{g} \in B$ (where $\operatorname{Jac}(\gamma)$ is the Jacobi matrix of $\gamma$ ), and hence

$$
\gamma(p)=\gamma(f u+g)=q(x)\left[x \hat{f}(x, y) u+\tilde{g}_{0}(x, y) y^{2}+\hat{g}_{1}(x, y) z\right]+a_{0}(x)+a_{1}(x) y .
$$

In view of the equality of ideals

$$
\left(\hat{g}_{1}, x \hat{f}\right)=\left(\tilde{g}_{1}, \tilde{f}\right),
$$

for the intersection indices we have

$$
\hat{g}_{1}^{*}(0) \cdot(x \hat{f})^{*}(0)=\tilde{g}_{1}^{*}(0) \cdot \tilde{f}^{*}(0)=n,
$$


therefore

Now by 4.12

$$
\hat{g}_{1}^{*}(0) \cdot \hat{f}^{*}(0) \leq n-1
$$

$$
\hat{p}:=q(x)\left[\hat{f}(x, y) u+\tilde{g}_{0}(x, y) y^{2}+\hat{g}_{1}(x, y) z\right]+a_{0}(x)+a_{1}(x) y
$$

is an $x$-residual variable. Hence by the inductive hypothesis, it is also an $x$-variable. Again by 4.12, $\gamma(p)$ and $p=f u+g$ are as well. The induction step is done, so the proof is completed.

4.15. Proof of Theorem 4.2. By 3.21(v),(vi) we may suppose that $p=f u+g$ is an $x$-residual variable and $f_{\text {non-horiz }} \in \mathbb{C}[x]$ (cf. 4.4). Thus we must show that actually, under each of the assumptions (a)-(d), $p$ is an $x$-variable.

(a) (resp., (b)) immediately follows from 4.7 (resp., 4.14).

(c) We write the polynomial $p=f u+g$ in the form (34). The assumption $f_{\text {vert }}=1$ implies that $q^{-1}(0)=\emptyset$, and hence $\tilde{f}^{-1}(0) \cap \tilde{g}_{1}^{-1}(0)=\emptyset$. Now the conclusion follows from 4.13

(d) Since, by our assumption, $f_{\text {horiz }}=f_{\text {horiz }}^{\text {red }}$, we have $\tilde{f}=\tilde{f}^{\text {red }}$. In view of 4.12 we may suppose that $r=1$ in (34), and so $p$ can be written as follows:

$$
p=f u+g=q\left[\tilde{f} u+\tilde{g}_{0} y^{2}+\tilde{g}_{1} z+\tilde{f} \tilde{g}_{2} z^{2}\right]+a_{0}+a_{1} y .
$$

The $\mathbb{C}[x, y, z]$-automorphism $u \longmapsto u-z^{2} \tilde{g}_{2}(x, y, z)$ transforms $p$ into an $x$-residual variable of degree at most 1 in $z$. Now the conclusion follows from (b).

(e) From the form of $g$ one easily deduces that $f_{\text {horiz }}=f_{\text {horiz }}^{\text {red }}=1$ which is a special case of (d) or even (a).

The following result extends our list of variables $p=f u+g$ beyond those provided in 4.2 .

Proposition 4.16. Let $p=f u+g \in \mathbb{C}^{[4]}$ as in (34) be an x-residual variable. If $\tilde{g}_{1} \in \mathbb{C}[x]$ and $\tilde{g}_{2} z^{2}=\hat{g}_{2}\left(x, y, \tilde{g}_{1}(x) z\right) \in \mathbb{C}[x]\left[y, \tilde{g}_{1} z\right]$, then $p$ is an $x$-variable.

Proof. By 4.12 we may suppose that $r=1$ in (34), and so $p=\bar{p}\left(x, y, \tilde{g}_{1}(x) z, u\right)$, where the polynomial

$$
\bar{p}:=q\left[\tilde{f} u+\tilde{g}_{0} y^{2}+z+\tilde{f}^{\mathrm{red}} \hat{g}_{2}\right]+a_{0}+a_{1} y
$$

still is an $x$-residual variable. Indeed by our assumption for any $\lambda \in \mathbb{C} \backslash \tilde{g}_{1}^{-1}(0)$,

$$
\bar{p}_{\lambda}=p_{\lambda}\left(y, z / \tilde{g}_{1}(\lambda), u\right) \in \operatorname{Var}_{\mathbb{C}} \mathbb{C}^{[3]} .
$$

For $x_{0} \in g_{1}^{-1}(0)$, either $q\left(x_{0}\right)=0$ and then by the assumption, $\bar{p}_{x_{0}}=a_{0}\left(x_{0}\right)+$ $a_{1}\left(x_{0}\right) y=p_{x_{0}} \in \operatorname{Var}_{\mathbb{C}} \mathbb{C}^{[3]}$, or $q\left(x_{0}\right) \neq 0$ and then again, by 1.29(a), $\bar{p}_{x_{0}} \in$ $\operatorname{Var}_{B} B[z, u]$ with $B:=\mathbb{C}[y]$. It follows from 4.13 that the polynomial $\bar{p}$ as in (36) is an $x$-variable. Hence by [1.31(a) (with $z$ playing the role of $u$ ) $p=\bar{p}\left(x, y, \tilde{g}_{1}(x) z, u\right.$ ) is as well.

Example 4.17. The polynomial

$$
p:=y+x\left(x z+y\left(y u+x^{2} z^{2}\right)\right) \in \mathbb{C}^{[4]}
$$

is an $x$-variable. Indeed $p=\bar{p}(x, y, x z, u)$, where by $1.29(\mathrm{a}), \bar{p}:=y+x\left(z+y\left(y u+z^{2}\right)\right)$ is an $x$-residual variable (and hence by 4.13 is an $x$-variable). Therefore, $p$ is an $x$-residual variable as well, and so 4.16 applies. 
4.18. The Sathaye theorem on linear planes 34 cited in the introduction was generalized by D. Wright [41] for the embeddings $\mathbb{C}^{2} \hookrightarrow \mathbb{C}^{3}$ of the form $p=$ $f(x, y) u^{n}+g(x, y)=0$. Below is a similar result in dimension 4. It belongs to the second author and its proof can be found in [18].

Theorem 4.19. If $p=f(x, y, z) u^{n}+g(x, y, z) \in \mathbb{C}^{[4]}$ (with $n \geq 2$ ) is an x-residual variable then it is an $x$-variable.

4.20. To conclude, we would like to raise the following question. It concerns a (probably simplest) example of an $x$-residual variable of $\mathbb{C}^{[4]}$ which does not fit any of the assumptions 4.2 (a)-(d) or 4.16

Question. Is the polynomial $p=y+x\left(x z+y\left(y u+z^{2}\right)\right) \in \mathbb{C}^{[4]}$ a variable?

4.2. Simple modifications of $\mathbb{C}^{3}$ rectifiable in $\mathbb{C}^{5}$. The following definitions are inspired by [34].

4.21. Let $B$ be a commutative ring. We say that $p \in B^{[n]}$ is a $B$-hyperplane if $B^{[n]} /(p) \cong B^{[n-1]}$. We say that $p$ defines a $B$-hyperplane fibration if $p-\lambda$ is a $B$-hyperplane for every $\lambda \in B$.

If $B=\mathbb{C}$ or $B=\mathbb{C}[x]$ we simply say hyperplane, resp., $x$-hyperplane, instead of $B$-hyperplane.

Notice that an $x$-hyperplane $p\left(x, y_{1}, \ldots, y_{n}\right) \in \mathbb{C}[x]\left[y_{1}, \ldots, y_{n}\right]$ becomes a hyperplane $p_{x_{0}}\left(y_{1}, \ldots, y_{n}\right)$ for every fixed $x=x_{0} \in \mathbb{C}$. A polynomial $p$ with the latter property is called an $x$-residual hyperplane.

4.22. Observe [34, 20, 39] that a polynomial $p=f(x, y) u+g(x, y, z) \in \mathbb{C}^{[4]}$ is an $x$-residual plane if and only if it is an $x$-residual variable, and henceforth, if and only if it defines an $x$-residual plane fibration.

4.23. We say that two polynomials $p, q \in B\left[y_{1}, \ldots, y_{n}\right]$ are 1-stably equivalent if

$$
\gamma((p, v))=(q, v)
$$

for an automorphism $\gamma \in \operatorname{Aut}_{B} B\left[y_{1}, \ldots, y_{n}, v\right]$.

4.24. For instance, with this terminology 1.32 (b) says that $y+a p(y)$ with $a \in$ $B, p \in B[y]$ is 1 -stably equivalent to $y+p(a y)$, and moreover if $a^{k} \mid p(0)$, then $y+a p(y)$ is 1-stably equivalent to $y+p\left(a^{k+1} y\right) / a^{k}$.

Theorem 4.25. Let $p=f(x, y) u+g(x, y, z) \in \mathbb{C}^{[4]}$ be an $x$-residual plane. Then it is 1-stably equivalent to an $x$-variable. Consequently, $p$ defines an $x$-plane fibration, and each fiber $X_{\lambda}:=\{p=\lambda\} \quad(\lambda \in \mathbb{C})$ of $p$ is a 3 -fold in $\mathbb{C}^{4}$ isomorphic to $\mathbb{C}^{3}$ which can be rectified in $\mathbb{C}^{5}$.

Remark 4.26. Notice that 4.25]provides yet another proof of the implication (iii) $\Rightarrow$ (i) of 3.21 which does not need 1.7. Indeed, it proves $(\mathrm{v}) \Rightarrow(\mathrm{i})$ while (iii) $\Rightarrow(\mathrm{v})$ is observed in 4.22 above.

In the proof of 4.25 (see 4.28 below) we use the following lemma.

Lemma 4.27. If $p \in \mathbb{C}[x, y, z, u] \backslash \mathbb{C}[x, y]$ is an $x$-residual plane, then it is 1 stably equivalent (under an automorphism $\gamma \in \operatorname{Aut}_{B} B[y, v]$ with $B:=\mathbb{C}[x, z, u]$ ) to a polynomial $\bar{p} \in \mathbb{C}[x][y, z, u]$ which is still an $x$-residual plane and such that for every $x_{0} \in \mathbb{C}, \quad \bar{p}_{x_{0}}(y, z, u) \notin \mathbb{C}^{*} y+\mathbb{C}$. 
Proof. As $p \notin \mathbb{C}[x, y]$, the subset

$$
L(p):=\left\{x_{0} \in \mathbb{C} \mid p_{x_{0}} \in \mathbb{C}^{*} y+\mathbb{C}\right\}=:\left\{x_{1}, \ldots, x_{n}\right\} \subseteq \mathbb{C}
$$

is finite. The proof proceeds by a descending induction on $n=\operatorname{card} L(p)$.

If card $L(p)=0$ there is nothing to prove. Suppose that $n>0$, and let $x_{n}=0$. Then there exists an affine automorphism $\tau \in \operatorname{Aut}_{\mathbb{C}[x]} \mathbb{C}[x][y] \subseteq \operatorname{Aut}_{D} D[y]$ (where $D:=\mathbb{C}[x, z, u, v])$ such that $\tau(p)=y+x \hat{p}$, where $\hat{p} \in \mathbb{C}[x, y, z, u]$ and $\hat{p}(x, 0,0,0)=$ 0 . Indeed, up to an affine automorphism of $\mathbb{C}[y]$ we may assume that $p(0, y, z, u)=$ $y$, i.e., $p=y+x \tilde{p}$ with $\tilde{p} \in \mathbb{C}^{[4]}$, and then we apply the shift $y \longmapsto y-x \tilde{p}(x, 0,0,0)$ to obtain $\tau(p)=y+x \hat{p}$ with $\hat{p}:=\tilde{p}-\tilde{p}(x, 0,0,0)$.

The polynomial $\hat{p} \in \mathbb{C}^{[4]}$ admits a presentation:

$$
\hat{p}=y q_{1}(x, y, z, u)+x^{k}\left(x q_{2}(x, z, u)+q_{3}(z, u)\right) .
$$

As by our assumption for every fixed $x=x_{0} \in \mathbb{C}$ the polynomial $\tau(p)_{x_{0}}=y+$ $x_{0} \hat{p}_{x_{0}} \notin \mathbb{C}[y]$ is irreducible, we have $x q_{2}+q_{3} \neq 0$, and thereby $q_{3} \neq 0$. Furthermore,

$$
0=\hat{p}(x, 0,0,0)=x^{k}\left(x q_{2}(x, 0,0)+q_{3}(0,0)\right)=q_{3}(0,0),
$$

hence $q_{3} \notin \mathbb{C}$. Now by 1.32 (b) (cf. 4.24 above) there exists an automorphism $\alpha \in \operatorname{Aut}_{B} B[y, v]$ (with $B=\mathbb{C}[x, z, u]$ ) such that

$$
\alpha((y+x \hat{p}, v))=\left(y+\frac{\hat{p}\left(x, x^{k+1} y, z, u\right)}{x^{k}}, v\right) .
$$

Letting $\gamma_{n}:=\alpha \tau$ we obtain

$$
\gamma_{n}((p, v))=\left(p_{n}, v\right),
$$

where $p_{n}:=y+\hat{p}\left(x, x^{k+1} y, z, u\right) / x^{k}$. We have

$$
\begin{aligned}
p_{n} & =y+\frac{x^{k+1} y q_{1}\left(x, x^{k+1} y, z, u\right)+x^{k}\left(x q_{2}(x, z, u)+q_{3}(z, u)\right)}{x^{k}} \\
& =y+x y q_{1}\left(x, x^{k+1} y, z, u\right)+x q_{2}(x, z, u)+q_{3}(z, u) .
\end{aligned}
$$

Hence $p_{n}(0, y, z, u)=y+q_{3}(z, u) \in \operatorname{Var}_{\mathbb{C}} \mathbb{C}[y, z, u]$ with $q_{3} \notin \mathbb{C}$, and so $p_{n}(0, y, z, u) \notin$ $\mathbb{C}^{*} y+\mathbb{C}$. In other words,

$$
0=x_{n} \notin L\left(p_{n}\right):=\left\{\lambda \in \mathbb{C} \mid\left(p_{n}\right)_{\lambda} \in \mathbb{C}^{*} y+\mathbb{C}\right\} \subseteq \mathbb{C} .
$$

Now the induction step and the proof of the lemma are completed by the following

Claim. (a) $L\left(p_{n}\right)=\left\{x_{1}, \ldots, x_{n-1}\right\}=L(p) \backslash\left\{x_{n}\right\}$.

(b) Furthermore for any $x_{0} \neq 0,\left(p_{n}\right)_{x_{0}} \in \mathbb{C}[y, z, u]$ is a plane.

Proof of the Claim 15 It is enough to show that for every $x_{0} \neq 0$ there exists an affine automorphism $\beta_{x_{0}} \in \operatorname{Aut}_{\mathbb{C}} \mathbb{C}[y] \subseteq \operatorname{Aut}_{\mathbb{C}[z, u]} \mathbb{C}[z, u][y]$ such that

$$
\beta_{x_{0}}\left(p_{x_{0}}\right)=x_{0}^{k+1}\left(p_{n}\right)_{x_{0}} \text {. }
$$

Indeed, we have

$$
x_{0}^{k+1}\left(p_{n}\right)_{x_{0}}=x_{0}^{k+1} y+x_{0} \hat{p}_{x_{0}}\left(x_{0}^{k+1} y, z, u\right) .
$$

${ }^{15}$ Alternatively, (b) also follows from existence of a $\mathbb{C}[x]$-isomorphism $\mathbb{C}[x]^{[3]} /(p) \cong \mathbb{C}[x]^{[3]} /(\bar{p})$; see 1.33 (b). 
Consider the linear automorphism $\sigma_{x_{0}}: y \longmapsto x_{0}^{k+1} y$ extended to $\mathbb{C}[z, u][y]$ in a natural way. From (38) we get

$$
x_{0}^{k+1}\left(p_{n}\right)_{x_{0}}=\sigma_{x_{0}}\left(y+x_{0} \hat{p}_{x_{0}}(y, z, u)\right)=\sigma_{x_{0}}\left((\tau(p))_{x_{0}}\right)=\sigma_{x_{0}}\left(\left(\tau_{x_{0}}\left(p_{x_{0}}\right)\right)\right),
$$

where $\tau_{x_{0}}$ denotes the specialization of $\tau$ at $x_{0}$. Thus we obtain (37) with $\beta_{x_{0}}:=$ $\sigma_{x_{0}} \tau_{x_{0}} \in$ Aut $_{\mathbb{C}} \mathbb{C}[y]$ extended to $\mathbb{C}[z, u][y]$. Hence $p_{x_{0}} \in \mathbb{C}^{*} y+\mathbb{C}$ if and only if $\left(p_{n}\right)_{x_{0}}$ is also.

4.28. Proof of Theorem 4.25. If $f=0$, then by 4.6(c) and the Abhyankar-MohSuzuki Theorem, $p$ itself is an $x$-variable.

If $f \neq 0$, then by 4.27 $p=f u+g$ is 1 -stably equivalent to a polynomial $\bar{p} \in \mathbb{C}^{[4]}$ such that

$$
L(\bar{p}):=\left\{\lambda \in \mathbb{C} \mid \bar{p}_{\lambda} \in \mathbb{C}^{*} y+\mathbb{C}\right\}=\emptyset .
$$

It is easily seen that the polynomial $\bar{p}$ as constructed in the proof of 4.27 still has the form $\bar{p}=\bar{f} u+\bar{g}$ with $\bar{f} \in \mathbb{C}[x, y]$ and $\bar{g} \in \mathbb{C}[x, y, z]$. It follows from (39) that $\bar{f}_{\text {vert }}=1$. As $\bar{p}$ is also an $x$-residual plane, by [4.2(c) it is an $x$-variable. Consequently,

$$
\begin{aligned}
\mathbb{C}[x][y, z, u] /(p) & \cong \mathbb{C}[x][y, z, u, v] /(p, v) \cong \mathbb{C}[x][y, z, u, v] /(\bar{p}, v) \\
& \cong \mathbb{C}[x][y, z, u, v] /(u, v) \cong \mathbb{C}[x]^{[2]}
\end{aligned}
$$

Hence $p$ is an $x$-plane. In view of 4.22 the same arguments work for every 'fiber' $p-\lambda$ of $p=f u+g$ (with $\lambda \in \mathbb{C}[x])$. Therefore $p=f u+g$ defines an $x$-plane fibration.

\section{REFERENCES}

[1] S.S. Abhyankar, T.T. Moh, Embedding of the line in the plane, J. Reine Angew. Math. 276 (1975), 148-166. MR 52:407

[2] H. Bass, E.H. Connell, and D.L. Wright. Locally polynomial algebras are symmetric algebras, Invent. Math. 38:3 (1976/77), 279-299. MR 55:5613

[3] E. Begle, Duality theorems for generalized manifolds, Amer. J. Math. 67 (1945), 59-70. MR 6:182c

[4] A.D.R. Choudary, A. Dimca, Complex hypersurfaces diffeomorphic to affine spaces, Kodai Math. J. 17 (1994), 171-178. MR 95f:14084

[5] H. Derksen, Constructive Invariant Theory and the Linearization Problem, Ph.D. thesis, Basel, 1997.

[6] A. Dold, Lectures on algebraic topology, Springer, Berlin, 1974. Second edition MR 82c:55001

[7] I.V. Dolgachev, B.Ju. Veisfeiler, Unipotent group schemes over integral rings, Math. USSR, Izv. 8 (1975), 761-800.

[8] E. Edo, S. Vénéreau, Length 2 variables of $A[x, y]$ and transfer, Ann. Polinici Math., Proc. of the conf. Polynomial automorphisms and related topics, Cracovia, July 1999 (2001), 67-76. MR 2002f: 14080

[9] A. T. Fomenko and D. B. Fuks, A course in homotopic topology (Russian), "Nauka", Moscow, 1989. MR 92a:55001

[10] T. Fujita, On the topology of non-complete algebraic surfaces, J. Fac. Sci. Univ. Tokyo, Sect. IA 29 (1982), 503-566. MR 84g:14035

[11] D. B. Fuks, O. Ya. Viro, Homology and cohomology, in: Encyclopedia of Math. Sci. Vol. 24, Topology-2, VINITI, Moscow, 1988, 123-245 (in Russian).

[12] M. Gizatullin, On affine surfaces that can be completed by a nonsingular rational curve, Math. USSR, Izv. 4 (1970), 787-810. 
[13] S. Kaliman, Exotic analytic structures and Eisenman intrinsic measures, Israel Math. J. 88 (1994), 411-423. MR 95j:32038

[14] S. Kaliman, Polynomials with general $\mathbb{C}^{2}$-fibers are variables, Pacific J. Math. 203:1, 161189. MR 2003d:14078

[15] S. Kaliman, L. Makar-Limanov, On Russell-Koras contractible threefolds, J. of Algebraic Geom. 6 (1997), 247-268. MR 98m:14041

[16] S. Kaliman, L. Makar-Limanov, Locally nilpotent derivations of Jacobian type, Preprint, 1998, 16pp.

[17] S. Kaliman, S. Vénéreau, M. Zaidenberg, Extensions birationnelles simples de l'anneau de polynômes $\mathbb{C}^{3}$, C. R. Acad. Sci. Paris Sér. I Math. 333:4 (2001), 319-322.

[18] S. Kaliman, S. Vénéreau, M. Zaidenberg, Simple birational extensions of the polynomial ring $\mathbb{C}^{[3]}$, preprint, Institut Fourier des mathématiques, 532; E-print math.AG/0104204 (2001), 49pp.

[19] S. Kaliman, M. Zaidenberg, Affine modifications and affine varieties with a very transitive automorphism group, Transformation Groups 4:1 (1999), 53-95. MR 2000f:14099

[20] S. Kaliman, M. Zaidenberg, Families of affine planes: the existence of a cylinder, Michigan Math. J. 49:2 (2001), 353-367. MR 2002e:14106

[21] S. Kaliman, M. Zaidenberg, Miyanishi's characterization of the affine 3-space does not hold in higher dimensions, Annales de l'Institut Fourier 50:6 (2000), 1649-1669. MR 2002c:14094

[22] V.Ya. Lin, M.G. Zaidenberg, Finiteness theorems for holomorphic mappings, Encyclopedia of Math. Sci. Vol. 9. Several Complex Variables III. Berlin, Heidelberg, New York: Springer Verlag, 1989, 113-172.

[23] L. Makar-Limanov, On the hypersurface $x+x^{2} y+z^{2}+t^{3}=0$ in $\mathbb{C}^{4}$ or a $\mathbb{C}^{3}$-like threefold which is not $\mathbb{C}^{3}$, Israel J. Math. 96 (1996), 419-429. MR 98a:14052

[24] L. Makar-Limanov, Again $x+x^{2} y+z^{2}+t^{3}=0$, Preprint, 1998, 3p.

[25] L. Makar-Limanov, Locally nilpotent derivations of affine domains, Abstract in: Affine Algebraic Geometry 14.05-20.05.2000, Mathematisches Forschungsinstitut Oberwolfach, Tagungsbericht $21 / 2000$, p. 11.

[26] W.S. Massey, Singular Homology Theory, Springer, New York, 1980. MR 81g:55002

[27] C.R.F. Maunder, Algebraic topology, The New University Mathematics Series. Van Nostrand Reinhold Company Ltd., London, 1970.

[28] M. Miyanishi, An algebro-topological characterization of the affine space of dimension three, Amer. J. Math. 106 (1984), 1469-1485. MR 86a:14040

[29] M. Miyanishi, Algebraic characterization of the affine 3-space, Proc. Algebraic Geom. Seminar, Singapore, World Scientific, 1987, 53-67. MR 90a:14059

[30] M. Miyanishi, Open algebraic surfaces, CRM Monograph Series 12, AMS Providence, RI, 2001. MR 2002e:14101

[31] R. Rentschler, Opérations du groupe additif sur le plane affine, C.R. Acad. Sci. Paris 267 (1968), 384-387. MR 38:1093

[32] P. Russell, Simple birational extensions of two dimensional affine rational domains, Compos. Math. 33 (1976), 197-208. MR 55:2943

[33] P. Russell, A. Sathaye, On finding and cancelling variables in $k[X, Y, Z]$, J. Algebra 57 (1979), 151-166. MR 80j:14030

[34] A. Sathaye, On linear planes, Proc. Amer. Math. Soc. 56 (1976), 1-7. MR 53:13227

[35] A. Sathaye, Polynomial ring in two variables over a D. V. R.: A criterion, Invent. Math. 74 (1983), 159-168. MR 85j:14098

[36] V. Shpilrain, Jie-Tai Yu, Embeddings of hypersurfaces in affine spaces, E-print math.AG/0010211, 2000, 11p.; J. Algebra 239 (2001), 161-173. MR 2002m:14051

[37] V. Shpilrain, Jie-Tai Yu, Embeddings of curves in the plane, J. Algebra 217 (1999), 668-678. MR 2001f: 13012

[38] M. Suzuki, Propiétés topologiques des polynômes de deux variables complexes, et automorphismes algébriques de l'espace $\mathbb{C}^{2}$, J. Math. Soc. Japan 26 (1974), 241-257. MR 49:3188

[39] S. Vénéreau, Quelques automorphismes et variables de $A[x, y]$, preprint, Institut Fourier des mathématiques, 460 (1999), 10pp.

[40] R.L. Wilder, Topology of manifolds, Repr. of the 1963 ed. Amer. Mathem. Soc. Colloquium Publications, 32. Providence, Rhode Island, 1979. MR 82a:57001

[41] D. Wright, Cancellation of variables of the form $b T^{n}-a$, J. Algebra 52 (1978), 94-100. MR 58:709 
[42] M. Zaidenberg, Isotrivial families of curves on affine surfaces and characterization of the affine plane, Math. USSR Izvestiya 30:3 (1988), 503-532. Additions and corrections, ibid., 38:2 (1992), 435-437. MR 88k:14021

[43] M. Zaidenberg, On exotic algebraic structures on affine spaces, Algebra and Analysis. St. Petersbourg Mathem. J. 10:5 (1999), 3-73. MR 2001d:14069

Department of Mathematics, University of Miami, Coral Gables, Florida 33124

E-mail address: kaliman@math.miami.edu

Université Grenoble I, Institut Fourier, UMR 5582 CNRS-UJF, BP 74, 38402 St. MARTIN D'HÈres CÉDEX, FranCE

E-mail address: venereau@math.mcgill.ca

Université Grenoble I, Institut Fourier, UMR 5582 CNRS-UJF, BP 74, 38402 St. Martin D'Hères CÉDEX, France

E-mail address: zaidenbe@ujf-grenoble.fr 